NASA Technical Memorandum 102664

\title{
Three-dimensional Simulation of Vortex Breakdown
}

G. Kuruvila and M. D. Salas

June 1990

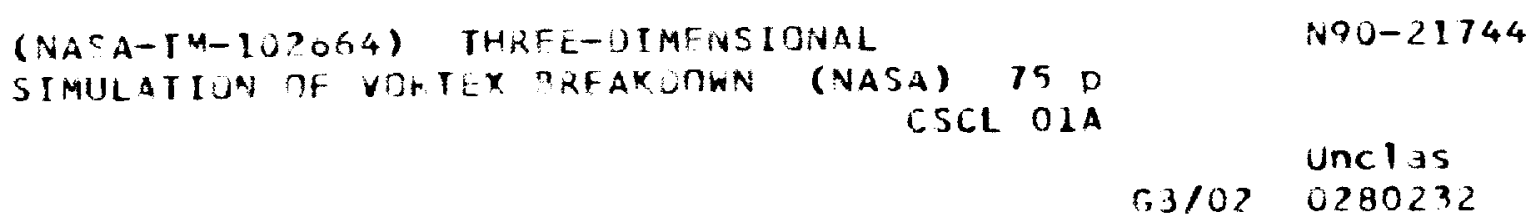




\section{Contents}

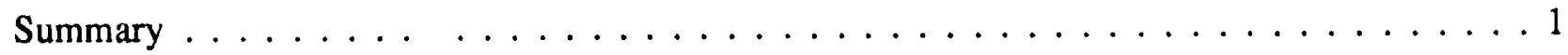

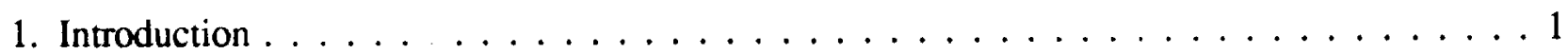

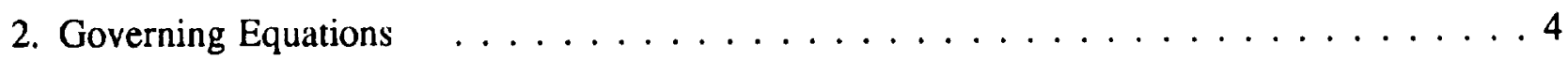

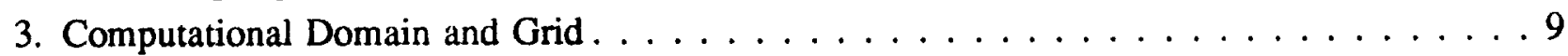

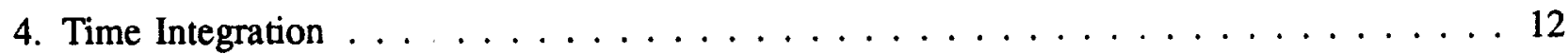

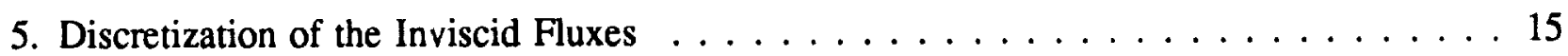

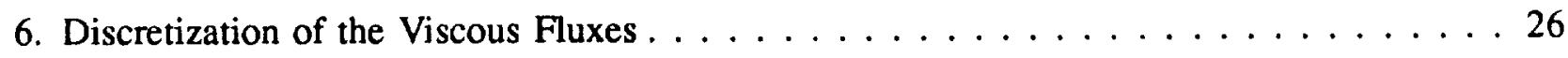

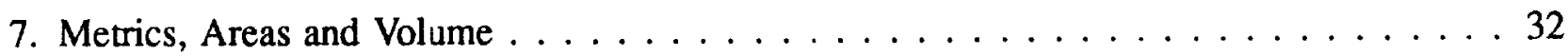

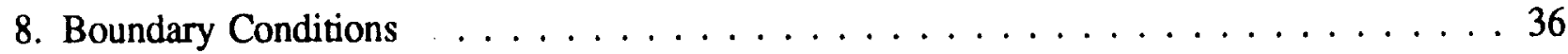

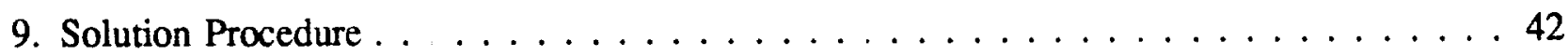

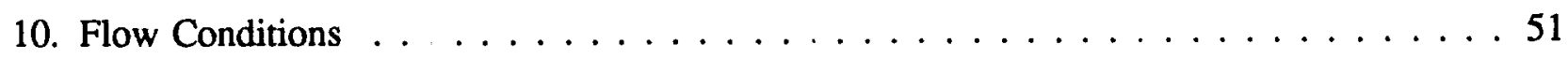

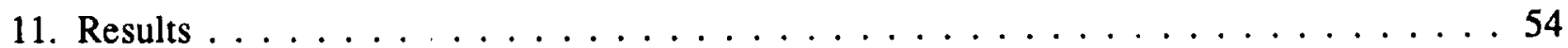

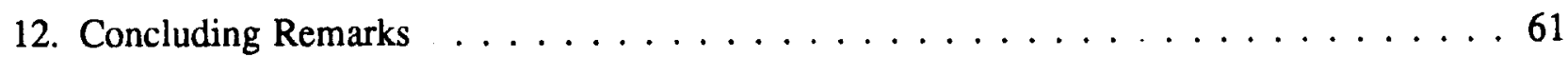

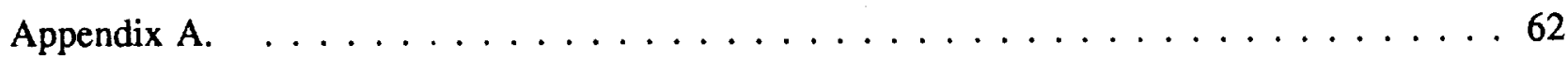

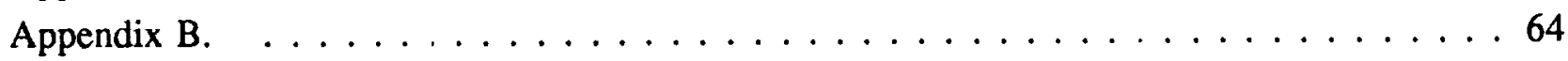

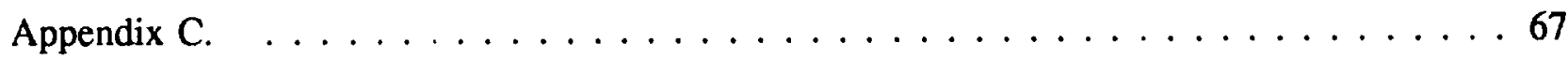

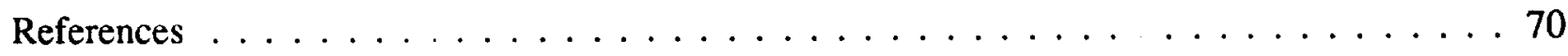





\section{Summary}

The integral form of the complete, unsteady, compressible, three-dimensional Navier-Stokes equations in the conservation form, cast in generalized coordinate system, are solved, numerically, to simulate the vortex breakdown phenomenon. The inviscid fluxes are spatially discretized using Roe's upwind-biased flux-difference splitting scheme and the viscous fluxes are discretized using central differencing. Time integration is performed using a backward Euler ADI scheme. A full approximation multigrid is used to accelerate the convergence to steady state.

\section{Introduction}

The phenomenon of "vortex bursting" or "vortex breakdown" was first reported by Peckham and Atkinson ${ }^{[1]}$ in 1957. They observed that under certain conditions, the vortex core shed by the leading edge of the Gothic wing they were testing would swell, eventually stagnating the flow along the vortex axis forming a bubble of recirculating flow. In the following years, similar observations were made by Elle ${ }^{[2][3]}$, Werle ${ }^{[4]}$, and Lambourne and Bryer ${ }^{[5]}$. The classic photograph in reference 5, reproduced here as Fig. 1-1, shows the sudden breakdown of the leading-edge vortices, once a critical angle of attack is reached, to either a symmetric bubble-type structure or an asymmetric spiral-type structure. Downstream of this structural change, the flow is usually highly turbulent and diffusive.

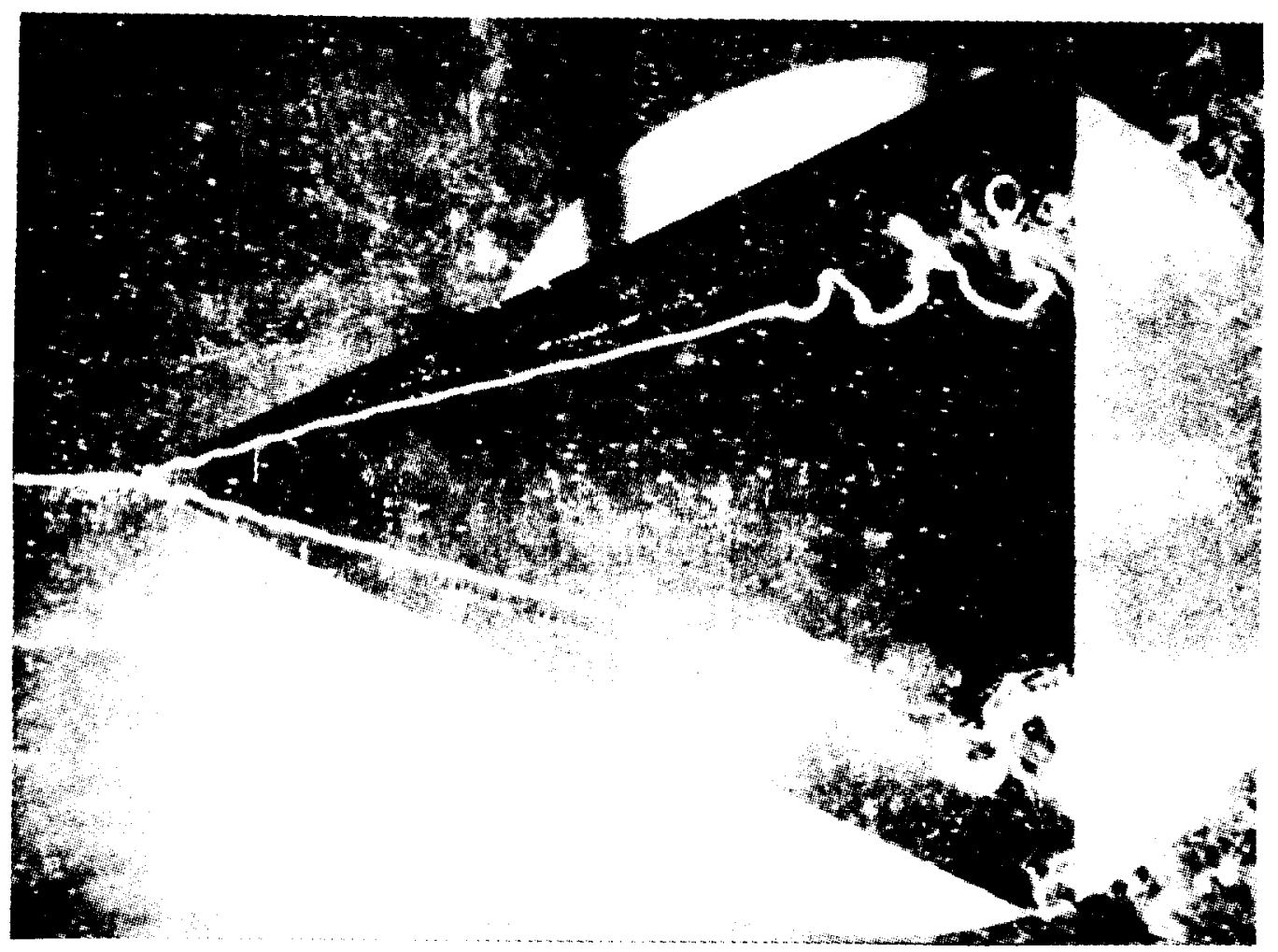

Figure 1-1. Leading-edge Vortex Breakdown (Lambourne and Bryer 1961) 
The effect of vortex breakdown on the aerodynamics of a wing is very important. Hummel and Srinivasan ${ }^{[6]}$ showed that, with increasing angle of attack, the lift, the drag and the pitching moment of a delta wing deteriorates abruptly as the vortex breakdown location moved upstream over the trailing edge of the wing. Peake and Tobak ${ }^{[7]}$ pointed out that vortex breakdown causes buffeting, unsteadiness and poor control. Modern fighter aircraft, like the F-18, that depend on vortex-induced lift for high-alpha maneuvers, experience significant loss of performance -loss of lift increase in drag and an increase in nose-up pitching moment- at certain attitudes, due to vortex breakdown. The fatigue life of some of the aircraft components, like the vertical stabilizers, are drastically reduced due to vibrations caused by vortex breakdown. Vortex breakdown is also known to cause undesirable effects in turbomachinery.

Some uses of vortex breakdown have also been suggested. Vortex breakdown may be used to dissipate the strong trailing vortices generated by large aircraft. The zone of recirculation flow of a vortex that has undergone breakdown can be used as a flame holder in gas turbine combustion chambers. Fuel and air can undergo intense turbulent mixing in the recirculating zone and result in a stable and compact flame. This will help increase combustion efficiency, reduce the combustor size and control the pollutants ${ }^{[8]}$. However the importance of vortex breakdown to aircraft designed to do high-alpha maneuvers is the main motivation of NASA to understand this phenomenon.

The phenomenon of vortex breakdown has been studied, both experimentally and theoretically, for over 30 years. The most important contribution to the experimental study of vortex breakdown was made by Harvey ${ }^{[9]}$, who isolated the vortex from the wing. He successfully simulated vortex breakdown in tube flow with swirl introduced upstream by a system of guide vanes. This set the stage for most of the "clean" experimental work that followed. Theoretically, there had been many efforts to explain the bubble-type vortex breakdown. Although many theories have been proposed, none of them explain, satisfactorily, all the details of breakdown. The prevelent theory for the onset of breakdown is principally due to Benjamin ${ }^{[10]}$. In Benjamin's theory, breakdown is explained as a transition between a supercritical upstream flow incapable of supporting upstream propagating waves and a subcritical flow which allows upstream and downstream propagating waves. The theory is based on an inviscid quasi-cylindrical approximation which neglects all axial gradients. In general, no consistent correlation has been found between the occurence of breakdown in the numerical solutions of the Navier-Stokes equations and the criticality condition of Benjamin. Comprehensive reviews of the progress made in understanding and predicting vortex breakdown have been given by Hall ${ }^{[1]}$ and Leibovich ${ }^{[12][13]}$.

Numerical simulation of the bubble type breakdown of isolated vortex has been simulated by many researchers. Grabowski and Berger ${ }^{[14]}$, using Chorin's artificial compressibility method and a primitive variable formulation, calculated the first numerical solution of the steady axisymmetric Navier-Stokes equations for this problem. However, the numerical scheme had difficulties in realizing fully-converged solutions and obtaining solutions for Reynolds numbers, based on vortexcore radius, greater than 200 . Hafez et al. ${ }^{[15]}$, using upwind differences and vertical line relaxation, solved the Navier-Stokes equations using the streamfunction - vorticity formulation and confirmed the earlier results of Grabowski and Berger. Although they were able to obtain converged steady state solutions for low Reynolds numbers, they encountered the same difficulty as Grabowski and 
Berger beyond a Reynolds number of 200 . Krause et al. ${ }^{[16]}$ and Menne ${ }^{[17]}$, used an Alternating Direction Implicit (ADI) procedure to solve the same equations as reference 15, but failed to obtain a steady state solution. In order to overcome the 200 Reynolds number barrier, Hafez et al. ${ }^{[18]}$, Salas and Kuruvila ${ }^{[19]}$ and Beren ${ }^{[20]}$, each working independently, attacked the problem using direct matrix inversion technique. In reference 19, the present authors give details of this scheme and report solutions obtained for Reynolds numbers as high as 1800 . Encouraged by the success of this technique, this effort was directed towards finding the effect of a perturbation on the spatial stability of these solutions and to eventually capture the spiral-type breakdown mode. The full three-dimensional Navier-Stokes equations in a cylindrical coordinate system, decomposed in the azimuthal-direction using Fourier transforms, was solved for the first two Fourier modes. In this study ${ }^{[21][22]}$ is was found that, at low Reynolds numbers, the effect of perturbation on the first non-axisymmetric Fouriel component is relatively small. However, the effect was significant at higher Reynolds numbers. Inclusion of higher modes, to validate these results, using this method, leads to a formidably large and expensive problem. Hence the present effort was directed towards solving the full three-dimensional problem using an iterative technique.

In this study the integral form of the unsteady compressible three-dimensional Navier-Stokes equation are discretized in space using the finite volume approach and integrated in time using an ADI scheme. The inviscicl components of the equations are discretized using Roe's flux-difference split upwind scheme and the viscous components are discretized using central difference. Multigrid acceleration is used to accelerate the convergence when the problem has a steady state solution. 


\section{Governing Equations}

The fundamental equations of fluid dynamics, the Navier-Stokes equations, that are based on the universal laws of mass, energy and momentum, a constitutive law defining the relationship between fluid properties and empirical laws stating the dependence of viscosity and thermal conductivity with other flow variables, completely describes all flow phenomenon. Assuming that there are no external forces and heat sources, the nondimensionalized Navier-Stokes equations in a Cartesian coordinate system can be written as

$$
\frac{\partial Q}{\partial t}+\frac{\partial(\tilde{E}-\tilde{\mathcal{E}})}{\partial x}+\frac{\partial(\tilde{F}-\tilde{\mathcal{F}})}{\partial y}+\frac{\partial(\tilde{G}-\tilde{\mathcal{G}})}{\partial z}=0
$$

where $Q$ is the vector of conserved variables, $\tilde{E}, \tilde{F}$ and $\tilde{G}$ are the vectors of Euler (advective) fluxes and $\tilde{\mathcal{E}}, \tilde{\mathcal{F}}$ and $\tilde{\mathcal{G}}$ are the vectors of viscous (diffusive) fluxes. The equations are arranged such that the first and second rows of the vector correspond to the continuity and the energy equations respectively, while the third, fourth and the fifth rows correspond to the momentum equations in the $x, y$ and $z$ directions respectively. Thus

$$
\begin{gathered}
Q=\left[\begin{array}{c}
\rho \\
\rho e \\
\rho u \\
\rho v \\
\rho w
\end{array}\right] \\
\tilde{E}=\left[\begin{array}{c}
\rho u \\
\rho u H \\
\rho u^{2}+p \\
\rho u v \\
\rho u w
\end{array}\right] \quad \tilde{\mathcal{E}}=\left[\begin{array}{c}
u \tau_{x x}+v \tau_{x y}+w \tau_{x z}-\dot{q_{x}} \\
\tau_{x x} \\
\tau_{x y} \\
\tau_{x z} \\
\\
\rho v \\
\rho v H \\
\rho u v \\
\rho v^{2}+p \\
\rho v w
\end{array}\right] \quad \tilde{\mathcal{F}}=\left[\begin{array}{c}
u \tau_{y x}+v \tau_{y y}+w \tau_{y z}-\dot{q}_{y} \\
\tau_{y x} \\
\tau_{y y} \\
\tau_{y z}
\end{array}\right]
\end{gathered}
$$




$$
\tilde{G}=\left[\begin{array}{c}
\rho w \\
\rho w H \\
\rho u w \\
\rho v w \\
\rho w^{2}+p
\end{array}\right] \quad \tilde{\mathcal{G}}=\left[\begin{array}{c}
0 \\
u \tau_{z x}+v \tau_{z y}+w \tau_{z z}-\dot{q}_{z} \\
\tau_{z x} \\
\tau_{z y} \\
\tau_{z z}
\end{array}\right]
$$

where $\rho$ is the density, $e$ is the total energy per unit mass, $u, v, w$ are the velocities in the $x, y, z$ directions respectively and $p$ is the pressure . $H$, the total enthalpy, is given by

$$
H=e+\frac{p}{\rho}
$$

$\tau_{i j}$ are the components of the shear-stress tensor, $\dot{q}_{i}$ are the components of the heat-flux vector and $t$ is the time. Assuming that the fluid is Newtonian and invoking the Stokes hypothesis, the components of the shear-stress tensor are given by

$$
\begin{gathered}
\tau_{x x}=\frac{2}{3} \frac{M_{\infty}}{R e} \mu\left(2 \frac{\partial u}{\partial x}-\frac{\partial v}{\partial y}-\frac{\partial w}{\partial z}\right) \\
\tau_{y y}=\frac{2}{3} \frac{M_{\infty}}{R e} \mu\left(2 \frac{\partial v}{\partial y}-\frac{\partial u}{\partial x}-\frac{\partial w}{\partial z}\right) \\
\tau_{z z}=\frac{2}{3} \frac{M_{\infty}}{R e} \mu\left(2 \frac{\partial w}{\partial z}-\frac{\partial u}{\partial x}-\frac{\partial v}{\partial y}\right) \\
\tau_{x y}=\tau_{y x}=\frac{M_{\infty}}{R e} \mu\left(\frac{\partial u}{\partial y}+\frac{\partial v}{\partial x}\right) \\
\tau_{x z}=\tau_{z x}=\frac{M_{\infty}}{R e} \mu\left(\frac{\partial w}{\partial x}+\frac{\partial u}{\partial z}\right) \\
\tau_{y z}=\tau_{z y}=\frac{M_{\infty}}{R e} \mu\left(\frac{\partial v}{\partial z}+\frac{\partial w}{\partial y}\right)
\end{gathered}
$$

and the components of the heat-flux vector are given by

$$
\dot{q}_{x}=-\frac{\gamma M_{\infty} \mu}{(\gamma-1) \operatorname{RePr}} \frac{\partial T}{\partial x}
$$




$$
\begin{aligned}
& \dot{q}_{y}=-\frac{\gamma M_{\infty} \mu}{(\gamma-1) \operatorname{RePr}} \frac{\partial T}{\partial y} \\
& \dot{q}_{z}=-\frac{\gamma M_{\infty} \mu}{(\gamma-1) \operatorname{RePr}} \frac{\partial T}{\partial z}
\end{aligned}
$$

where $\mu$ is the coefficient of molecular viscosity, $T$ is the temperature, $\gamma$ is the ratio of specific heats, $P r$ is the Prandtl number, $R e$ is the reference Reynolds number and $M_{\infty}$ is the reference Mach number.

The quantities used for nondimensionalization of the equations are a reference length $L$ and reference values of density $\rho_{\infty}$, speed of sound $a_{\infty}$, and viscosity $\mu_{\infty}$. Using these, the nondimensional quantities are

$$
\begin{aligned}
& x=\frac{\hat{x}}{L} \quad y=\frac{\hat{y}}{L} \quad z=\frac{\hat{z}}{L} \quad t=\frac{\hat{t}}{L / a_{\infty}} \\
& \rho=\frac{\hat{\rho}}{\rho_{\infty}} \quad e=\frac{\hat{e}}{a_{\infty}^{2}} \quad u=\frac{\hat{u}}{a_{\infty}} \quad v=\frac{\hat{v}}{a_{\infty}} \quad w=\frac{\hat{w}}{a_{\infty}} \\
& p=\frac{\hat{p}}{\rho_{\infty} a_{\infty}^{2}} \quad T=\frac{\hat{T}}{a_{\infty}^{2} / \mathfrak{R}} \quad H=\frac{\hat{H}}{a_{\infty}^{2}} \quad \mu=\frac{\hat{\mu}}{\mu_{\infty}}
\end{aligned}
$$

where the dimensional quantities are denoted by a hat and $\mathfrak{R}$ is the gas constant. The reference Reynolds number is given by

$$
R e=\frac{\rho_{\infty} U_{\infty} L}{\mu_{\infty}}
$$

and the reference Mach number is given by

$$
M_{\infty}=\frac{U_{\infty}}{a_{\infty}}
$$

where $U_{\infty}$ is a reference velocity. The Prandtl number is given by

$$
\operatorname{Pr}=\frac{\gamma \Re}{(\gamma-1)} \frac{\hat{\mu}}{\hat{k}}
$$


where $\hat{k}$ is the coefficient of thermal conductivity. The equation set is closed using a constitutive relation, the perfect gas equation of state

$$
p=(\gamma-1) \rho\left[e-\frac{1}{2}\left(u^{2}+v^{2}+w^{2}\right)\right]
$$

The coefficient of molecular viscosity is determined using the power law

$$
\mu=T^{\omega}
$$

where $\omega$ is a constant.

For convenience of discretizing, the governing equations are transformed from the physical domain $(x, y, z)$ to the computational domain $(\xi, \eta, \zeta)$ using the transformation

$$
\begin{aligned}
& \xi=\xi(x, y, z) \\
& \eta=\eta(x, y, z) \\
& \zeta=\zeta(x, y, z)
\end{aligned}
$$

Applying the chain rule of partial differentiation, the governing equations in the computational domain can be written as

$$
\frac{1}{j} \frac{\partial Q}{\partial t}+\frac{\partial(E-\mathcal{E})}{\partial \xi}+\frac{\partial(F-\mathcal{F})}{\partial \eta}+\frac{\partial(G-\mathcal{G})}{\partial \zeta}=0
$$

where

$$
\left[\begin{array}{l}
E-\mathcal{E} \\
F-\mathcal{F} \\
G-\mathcal{G}
\end{array}\right]=\frac{1}{J}\left[\begin{array}{lll}
|\nabla \xi| \bar{\xi}_{x} & |\nabla \xi| \bar{\xi}_{y} & |\nabla \xi| \bar{\xi}_{z} \\
|\nabla \eta| \bar{\eta}_{x} & |\nabla \eta| \bar{\eta}_{y} & |\nabla \eta| \bar{\eta}_{z} \\
|\nabla \zeta| \bar{\zeta}_{x} & |\nabla \zeta| \bar{\zeta}_{y} & |\nabla \zeta| \bar{\zeta}_{z}
\end{array}\right]\left[\begin{array}{c}
\tilde{E}-\tilde{\mathcal{E}} \\
\tilde{F}-\tilde{\mathcal{F}} \\
\tilde{G}-\tilde{\mathcal{G}}
\end{array}\right]
$$

where

$$
\left[\begin{array}{c}
\bar{r}_{x} \\
\bar{r}_{y} \\
\bar{r}_{z}
\end{array}\right]=\frac{1}{|\nabla r|}\left[\begin{array}{l}
r_{x} \\
r_{y} \\
r_{z}
\end{array}\right] \quad, \quad|\nabla r|=\left(r_{x}^{2}+r_{y}^{2}+r_{z}^{2}\right)^{1 / 2}
$$

where $r$ stands for $(\xi, \eta, \zeta)$. The metric terms associated with the coordinate transformation are 


$$
\begin{aligned}
& \xi_{x}=J\left(y_{\eta} z_{\zeta}-y_{\zeta} z_{\eta}\right) \\
& \xi_{y}=J\left(x_{\zeta} z_{\eta}-x_{\eta} z_{\zeta}\right) \\
& \xi_{z}=J\left(x_{\eta} y_{\zeta}-x_{\zeta} y_{\eta}\right) \\
& \eta_{x}=J\left(y_{\zeta} z_{\xi}-y_{\xi} z_{\zeta}\right) \\
& \eta_{y}=J\left(x_{\xi} z_{\zeta}-x_{\zeta} z_{\xi}\right) \\
& \eta_{z}=J\left(x_{\zeta} y_{\xi}-x_{\xi} y_{\zeta}\right) \\
& \zeta_{x}=J\left(y_{\xi} z_{\eta}-y_{\eta} z_{\xi}\right) \\
& \zeta_{y}=J\left(x_{\eta} z_{\xi}-x_{\xi} z_{\eta}\right) \\
& \zeta_{z}=J\left(x_{\xi} y_{\eta}-x_{\eta} y_{\xi}\right)
\end{aligned}
$$

where $J$, the Jacobian of the transformation is given by

$$
J=1 /\left[x_{\xi}\left(y_{\eta} z_{\zeta}-y_{\zeta} z_{\eta}\right)-x_{\eta}\left(y_{\xi} z_{\zeta}-y_{\zeta} z_{\xi}\right)+x_{\zeta}\left(y_{\xi} z_{\eta}-y_{\eta} z_{\xi}\right)\right]
$$

The metrics can be determined either from analytic expressions for the inverse of the transformation,

$$
\begin{aligned}
& x=x(\xi, \eta, \zeta) \\
& y=y(\xi, \eta, \zeta) \\
& z=z(\xi, \eta, \zeta)
\end{aligned}
$$

or numerically as a direct result of a grid generation scheme,

$$
\begin{aligned}
& x=x(i, j, k) \\
& y=y(i, j, k) \\
& z=z(i, j, k)
\end{aligned}
$$

In this study the metrics are evaluated, numerically, using the coordinates of the grid points. The grid generation scheme and the evaluation of the metrics will be discussed later. 


\section{Computational Domain and Grid}

In this study, the Navier-Stokes equations are solved, in a space in the shape of a cuboid as shown in Fig. 3-1. The boundaries of the computational domain are referred to in the following way. In Fig. 3-1, the left hand side face ( $y z$-plane at $x=0)$ is called the incoming-face and the opposite face is called the outgoing-face. The four faces around the $x$-axis are called the side-faces. An isolated vortex is introduced at the incoming face by specifying the profiles of the flow variables. The axis of the incoming vortex is aligned along the $x$-direction and it is centered on the incoming-face. The incoming- and outgoing- faces are squares. Note that the boundaries around the vortex are ps:rmeable.

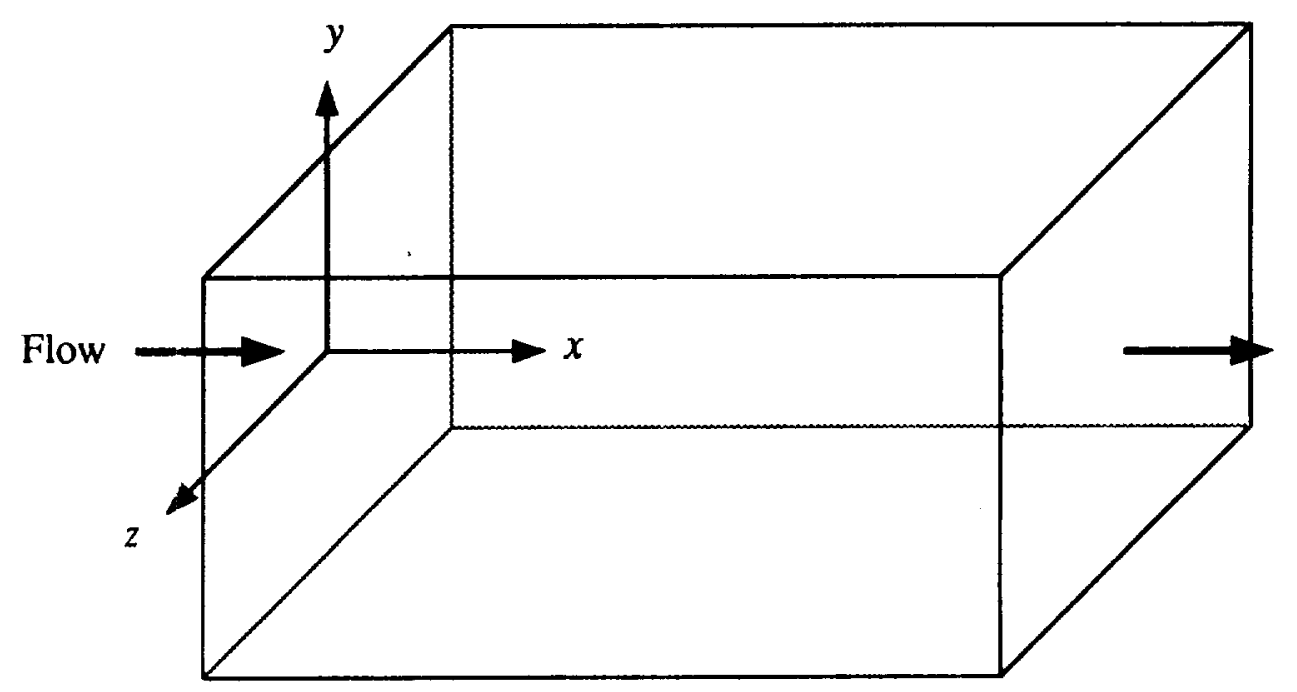

Figure 3-1. The Computational Domain

An algebraic grid that is uniform in the $x$-direction and stretched in the $y$-and $z$-directions is used. The grid in the $y$-direction, for example, is stretched using the equation

$$
y=y_{\max } \theta\left[\frac{\beta-1}{\beta-\theta}\right] \quad 0 \leq \theta \leq 1
$$

$\beta$ is a free parameter that is used to control the amount of stretching. Fig 3-2 shows the grid that is used in this study. It has $65 \times 33 \times 33$ grid points in a computational space that is $10 \times 8 \times 8$. All the cells in the flowfield are rectangular boxes. From the numerical grid generation scheme, we 
define,

$$
\begin{aligned}
& x=x\left(i \pm \frac{1}{2}, j \pm \frac{1}{2}, k \pm \frac{1}{2}\right) \\
& y=y\left(i \pm \frac{1}{2}, j \pm \frac{1}{2}, k \pm \frac{1}{2}\right) \\
& z=z\left(i \pm \frac{1}{2}, j \pm \frac{1}{2}, k \pm \frac{1}{2}\right)
\end{aligned}
$$

Eq. 3-2 specifies the coordinates of the discrete cells in the flowfield.

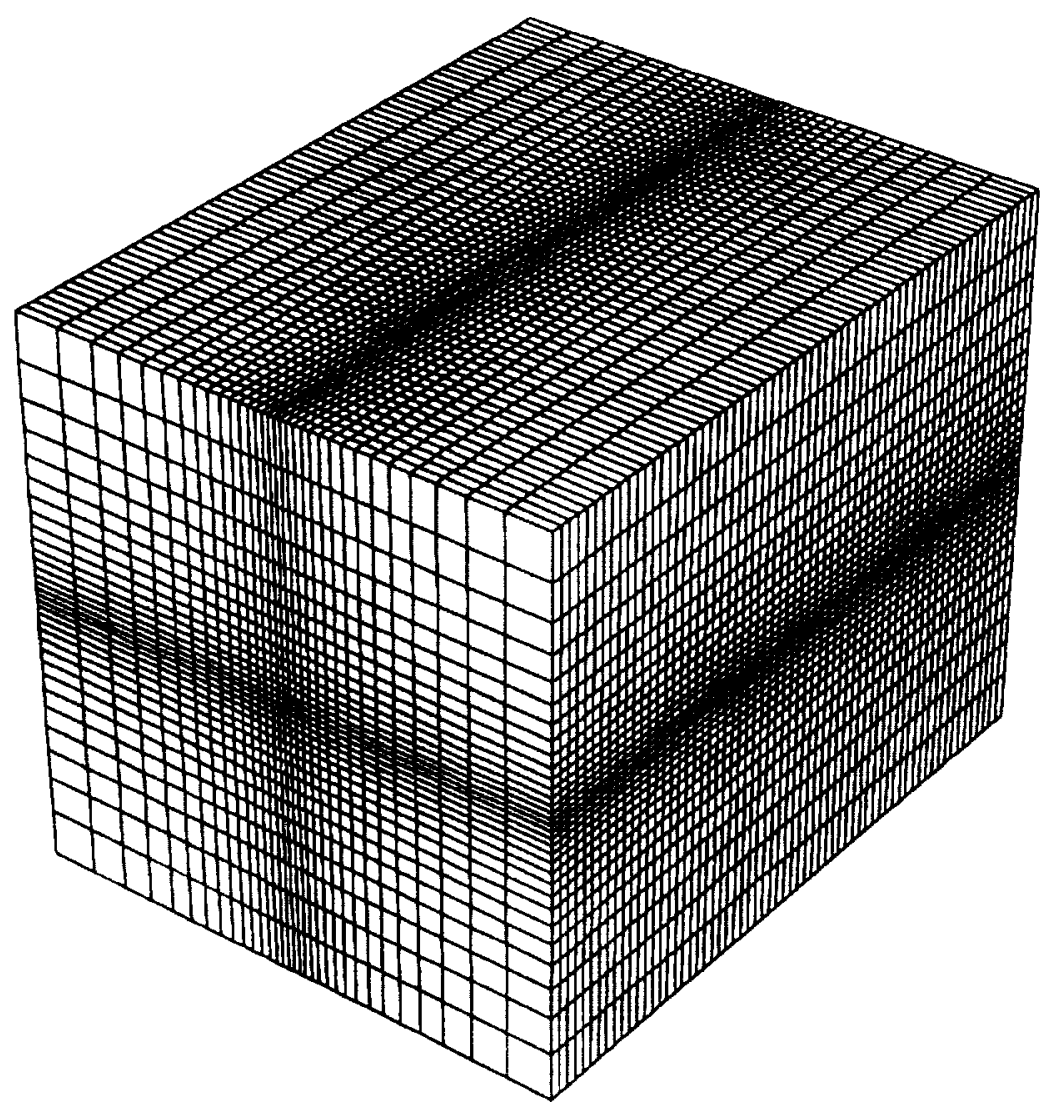

Figure 3-2. The Grid

For complex topologies a grid generation scheme produces cells that are, at best, hexahedrons. Consider a cell $(i, j, k)$ in the flowfield, shown in Fig. 3-3. Let $Q_{i, j, k}$, the conserved variables, be located at the cell-center. A semidiscrete finite-volume representation of the governing equations, 
Eq. 2-23, can be written as

$$
\frac{1}{J} \frac{\partial Q}{\partial t}+\frac{\Delta(E-\mathcal{E})}{\Delta \xi}+\frac{\Delta(F-\mathcal{F})}{\Delta \eta}+\frac{\Delta(G-\mathcal{G})}{\Delta \zeta}=0
$$

Choosing $\Delta \xi=\Delta \eta=\Delta \zeta=1$, the flux balance in a cell can be written as

$$
\begin{aligned}
\left(\frac{1}{J} \frac{\partial Q}{\partial t}\right)_{i, j, k} & +(E-\mathcal{E})_{i+\frac{1}{2}, j, k}-(E-\mathcal{E})_{i-\frac{1}{2}, j, k} \\
& +(F-\mathcal{F})_{i, j+\frac{1}{2}, k}-(F-\mathcal{F})_{i, j-\frac{1}{2}, k} \\
& +(G-\mathcal{G})_{i, j, k+\frac{1}{2}}-(G-\mathcal{G})_{i, j, k-\frac{1}{2}}=0
\end{aligned}
$$

where the conserved variables, located at the cell-center $(i, j, k)$, are cell averages and the fluxes are evaluated at the cell interfaces $i \pm \frac{1}{2}, j \pm \frac{1}{2}$ and $k \pm \frac{1}{2}$. It can be shown (Appendix-A) that Eq. 2-23 is a consistent approximation to the integral form of the governing equations.
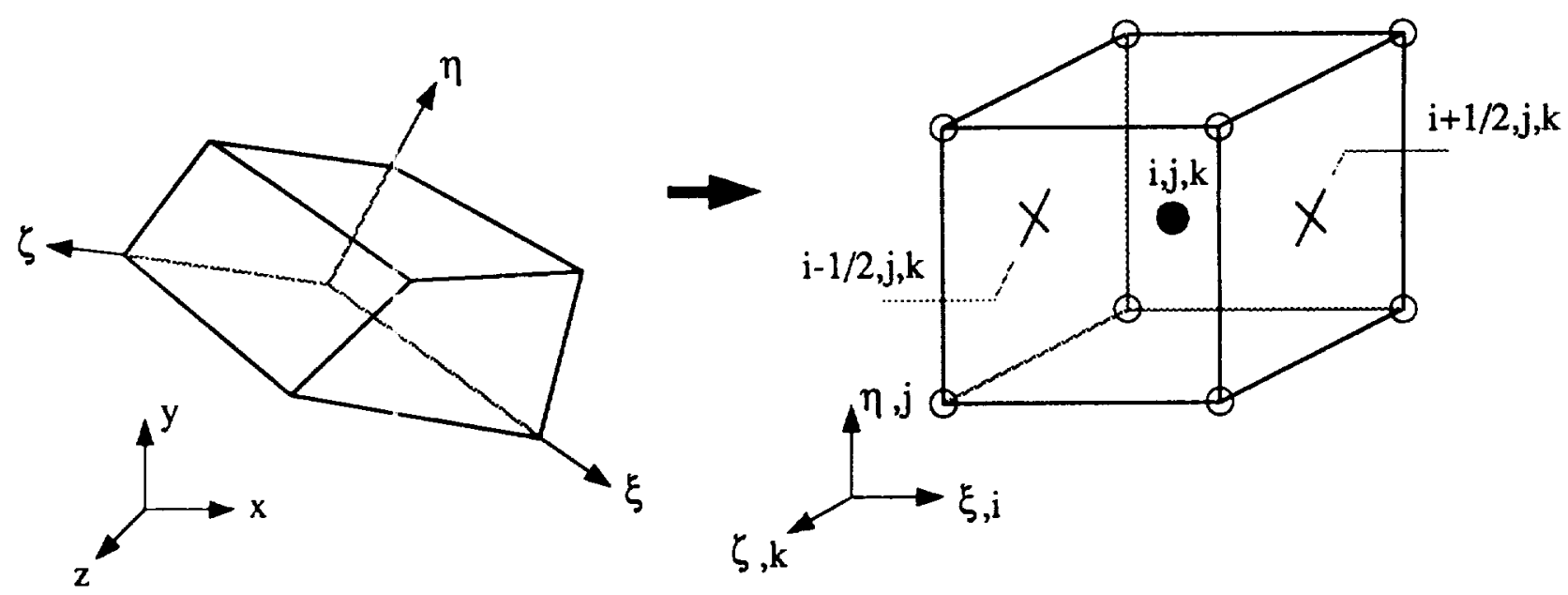

Location of Coordinates

Location of Variables

$\times$ Cell Interface where fluxes are evaluated

Figure 3-3. A Cell in the Flowfield 


\section{Time Integration}

We are interested in advancing the governing equations in time from some set of initial conditions. We are interested in the steady state solution if one exists or in the time evolution of the flowfield if the flow is unsteady. We choose an implicit scheme, developed by Beam and Warming ${ }^{[23]}$, to march the governing equations in time. The scheme is implemented as described below. The governing equations, Eq. $2-23$ at time level $n$ is

$$
\frac{1}{J}\left(\frac{\partial Q}{\partial t}\right)^{n}+\left\{\frac{\partial(E-\mathcal{E})}{\partial \xi}+\frac{\partial(F-\mathcal{F})}{\partial \eta}+\frac{\partial(G-\mathcal{G})}{\partial \zeta}\right\}^{n}=0
$$

The solution $Q^{n+1}$ at the next time level, $n+1$ can be evaluated using the backward Euler formula

$$
Q^{n+1}=Q^{n}+\Delta t\left(\frac{\partial Q}{\partial t}\right)^{n+1}
$$

where $\Delta t$ is the time increment. Substituting Eq. 4-2 in Eq. 4-1 we get

$$
\frac{1}{J} \frac{\Delta Q}{\Delta t}+\left\{\frac{\partial(E-\mathcal{E})}{\partial \xi}+\frac{\partial(F-\mathcal{F})}{\partial \eta}+\frac{\partial(G-\mathcal{G})}{\partial \zeta}\right\}^{n+1}=0
$$

where

$$
\Delta Q=Q^{n+1}-Q^{n}
$$

Using Taylor-series expansion we can write

$$
\begin{aligned}
& (E-\mathcal{E})^{n+1}=(E-\mathcal{E})^{n}+\left\{\frac{\partial(E-\mathcal{E})}{\partial Q}\right\}^{n} \Delta Q+\mathcal{O}\left(\Delta Q^{2}\right) \\
& (F-\mathcal{F})^{n+1}=(F-\mathcal{F})^{n}+\left\{\frac{\partial(F-\mathcal{F})}{\partial Q}\right\}^{n} \Delta Q+\mathcal{O}\left(\Delta Q^{2}\right) \\
& (G-\mathcal{G})^{n+1}=(G-\mathcal{G})^{n}+\left\{\frac{\partial(G-\mathcal{G})}{\partial Q}\right\}^{n} \Delta Q+\mathcal{O}\left(\Delta Q^{2}\right)
\end{aligned}
$$

Substituting Eq. 4-5 in Eq. 4-3 we get

$$
\begin{aligned}
{\left[\mathbf{I}+J \Delta t\left\{\delta_{\xi}\left(\frac{\partial E}{\partial Q}-\frac{\partial \mathcal{E}}{\partial Q}\right)^{n}+\delta_{\eta}\left(\frac{\partial F}{\partial Q}-\frac{\partial \mathcal{F}}{\partial Q}\right)^{n}+\delta_{\zeta}\left(\frac{\partial G}{\partial Q}\right.\right.\right.} & \left.\left.\left.-\frac{\partial \mathcal{G}}{\partial Q}\right)^{n}\right\}\right] \Delta Q \\
& =-J \Delta t\left(R^{n}-\mathcal{R}^{n}\right)
\end{aligned}
$$


where $I$ is an identity nuatrix, $\delta_{\xi}, \delta_{\eta}$ and $\delta_{\zeta}$ are difference operators in $\xi, \eta$ and $\zeta$ directions respectively,

$$
R^{n}=\left(\frac{\partial E}{\partial \xi}+\frac{\partial F}{\partial \eta}+\frac{\partial G}{\partial \zeta}\right)^{n}
$$

is the steady state residual of the inviscid part of the governing equations and

$$
\mathcal{R}^{n}=\left(\frac{\partial \mathcal{E}}{\partial \xi}+\frac{\partial \mathcal{F}}{\partial \eta}+\frac{\partial \mathcal{G}}{\partial \zeta}\right)^{n}
$$

is the steady state residual of the viscous part of the governing equations. If the flow has a steady state the total residual $R^{n}-\mathcal{R}^{n}$ goes to zero as time goes to infinity. The left hand side of Eq. 4-6 can be approximately factored as follows

$$
\begin{array}{r}
{\left[\mathrm{I}+J \Delta t \delta_{\xi}\left(\frac{\partial E}{\partial Q}-\frac{\partial \mathcal{E}}{\partial Q}\right)^{n}\right]\left[\mathrm{I}+J \Delta t \delta_{\eta}\left(\frac{\partial F}{\partial Q}-\frac{\partial \mathcal{F}}{\partial Q}\right)^{n}\right]\left[\mathrm{I}+J \Delta t \delta_{\zeta}\left(\frac{\partial G}{\partial Q}-\frac{\partial \mathcal{G}}{\partial Q}\right)^{n}\right] \Delta Q} \\
=-J \Delta t\left(R^{n}-\mathcal{R}^{n}\right)
\end{array}
$$

Eq. 4-9 can be replaced ty the following alternating direction sequence,

$$
\begin{aligned}
& {\left[\mathrm{J}+J \Delta t \delta_{\xi}\left(\frac{\partial E}{\partial Q}-\frac{\partial \mathcal{E}}{\partial Q}\right)^{n}\right] \Delta \mathcal{Q}=-J \Delta t\left(R^{n}-\mathcal{R}^{n}\right)} \\
& {\left[\mathrm{J}+J \Delta t \delta_{\eta}\left(\frac{\partial F}{\partial Q}-\frac{\partial \mathcal{F}}{\partial Q}\right)^{n}\right] \Delta \overline{\mathcal{Q}}=\Delta \mathcal{Q}} \\
& {\left[\mathrm{J}+J \Delta t \delta_{\zeta}\left(\frac{\partial G}{\partial Q}-\frac{\partial \mathcal{G}}{\partial Q}\right)^{n}\right] \Delta Q=\Delta \overline{\mathcal{Q}}}
\end{aligned}
$$

Each of these equations $i$ s: a set of block tri- or penta- diagonal equations depending on the spatial accuracy of the left hand side operator. These equations can be solved sequentially to obtain $\Delta Q$ from which the solution at the next time level can be obtained as

$$
Q^{n+1}=Q^{n}+\Delta Q
$$

The convergence rate of this scheme is sensitive to the factorization error. At large time steps, this error dominates the left hand side and the amplification factor of the error tends to unity. However the rate of convergence is relatively insensitive to the accuracy of the spatial differencing on either sicles of Eq. 4-10 ${ }^{[24]}$. Hence a first order accurate spatial differencing of 
the left hand side, that results in a block tri-diagonal system of equations, is a good choice since the computational work required is greatly reduced. However, higher order accurate spatial differencing is required on the right hand side for the accuracy of the solution.

\section{Spatial Differencing}

The sequence of equations, Eq. 4-10 are discretized in the following way. Dropping the superscript $n$ for convenience and neglecting higher order terms, Eq. 4-10a at a cell $i, j, k$ can be written as

$$
\begin{gathered}
\mathbf{I} \Delta Q_{i, j, k}+J \Delta t\left\{\left(\frac{\partial E}{\partial Q} \Delta Q-\frac{\partial \mathcal{E}}{\partial Q} \Delta Q\right)_{i+\frac{1}{2}, j, k}-\left(\frac{\partial E}{\partial Q} \Delta Q-\frac{\partial \mathcal{E}}{\partial Q} \Delta Q\right)_{i-\frac{1}{2}, j, k}\right\} \\
=-J \Delta t\left(R_{i, j, k}-\mathcal{R}_{i, j, k}\right)
\end{gathered}
$$

or

$$
\begin{gathered}
\mathbf{I} \Delta Q_{i, j, k}+J \Delta t \\
\left\{\left(\frac{\partial E}{\partial Q} \Delta Q\right)_{i+\frac{1}{2}, j, k}-\left(\frac{\partial E}{\partial Q} \Delta Q\right)_{i-\frac{1}{2}, j, k}-\left(\frac{\partial \mathcal{E}}{\partial Q} \Delta Q\right)_{i+\frac{1}{2}, j, k}+\left(\frac{\partial \mathcal{E}}{\partial Q} \Delta Q\right)_{i-\frac{1}{2}, j, k}\right\} \\
=-J \Delta t\left(E_{i+\frac{1}{2}, j, k}-E_{i-\frac{1}{2}, j, k}+F_{i, j+\frac{1}{2}, k}-F_{i, j-\frac{1}{2}, k}+G_{i, j, k+\frac{1}{2}}-G_{i, j, k-\frac{1}{2}}\right. \\
\left.-\mathcal{E}_{i+\frac{1}{2}, j, k}+\mathcal{E}_{i-\frac{1}{2}, j, k}-\mathcal{F}_{i, j+\frac{1}{2}, k}+\mathcal{F}_{i, j-\frac{1}{2}, k}-\mathcal{G}_{i, j, k+\frac{1}{2}}+\mathcal{G}_{i, j, k-\frac{1}{2}}\right)
\end{gathered}
$$

The left hand side of the other two equations, Eqs. 4-10b and 4-10c, in the sequence can be discretized in a similar way. The right hand side of these equations are obtained from the solution of the preceding equations respectively.

In the absence of external or internal sources, a flow configuration is the result of a balance between the inviscid (advective) fluxes, due to the convection of the flow, and the viscous fluxes due diffusion of the flow. Viscous fluxes influence the whole space domain and are independent of the flow direction while, the inviscid fluxes are dominated by waves, and information is transmitted through specific regions of space determined by the wave-propagation direction. Mathematically, the unsteady Navier-Stokes equations are parabolic when the flow is viscous dominated and hyperbolic if the viscous effects are negligible. This difference in the physical behavior between the inviscid and viscous fluxes has to be taken into account by the numerical discretization, in order to model the problem properly. In the next two sections we describe, how the flux vectors $E, F, G, \mathcal{E}, \mathcal{F}$ and $\mathcal{G}$ and the flux Jacobian matrices $\partial E / \partial Q, \partial F / \partial Q, \partial G / \partial Q, \partial \mathcal{E} / \partial Q, \partial \mathcal{F} / \partial Q$ and $\partial \mathcal{G} / \partial Q$ are evaluated at the cell interfaces. 


\section{Discretization of the Inviscid Fluxes}

In this section we outline the discretization of the inviscid fluxes using the Roe's flux-difference split upwind scheme. This scheme can be described using the one-dimensional Euler equations

$$
\frac{1}{J} \frac{\partial Q}{\partial t}+\frac{\partial E(Q)}{\partial \xi}=0
$$

which is a set of three equations. We seek a solution $Q(\xi, t)$ that satisfies Eq. 5-1 and the initial condition

$$
Q(\xi, 0)=Q_{0}(\xi)
$$

A semidiscrete finite-volume representation of Eq. 5-1 can be written as (Refer Eq. 3-3)

$$
\left(\frac{1}{J} \frac{\partial Q}{\partial t}\right)_{i}+E_{i+\frac{1}{2}}-E_{i-\frac{1}{2}}=0
$$

Once the fluxes $E$ at the cell interfaces $i+\frac{1}{2}$ and $i-\frac{1}{2}$ are evaluated as a function of $Q$, Eq. 5-3 can be integrated in time.

To determine the fluxes at the cell interface, we use Roe's flux-difference splitting upwind scheme ${ }^{[25]}$ Both analysis and numerical experiments have shown that this scheme provides a more accurate representation of shock waves and boundary layers than other conservative upwind schemes. However, the computational work required is slightly greater than that of other upwind schemes. Roe's scheme constructs the solution to Eq. 5-3 by solving an approximate Riemann problem at each of the cell interfaces. We begin by constructing a higher order interpolation ${ }^{[26]}$ of the state variable $Q$ on either sides of a cell interface, namely:

$$
\begin{aligned}
& \left(Q_{1}\right)_{i+\frac{1}{2}}=Q_{i}+\frac{1}{4}\left[(1+\kappa) \delta Q_{i}^{+}+(1-\kappa) \delta Q_{i}^{-}\right] \\
& \left(Q_{R}\right)_{i+\frac{1}{2}}=Q_{i+1}-\frac{1}{4}\left[(1+\kappa) \delta Q_{i+1}^{-}+(1-\kappa) \delta Q_{i+1}^{+}\right]
\end{aligned}
$$

where

$$
\delta Q_{i}^{+}=Q_{i+1}-Q_{i}
$$

and

$$
\delta Q_{i}^{-}=Q_{i}-Q_{i-1}
$$




\begin{tabular}{|c|c|c|}
\hline$\kappa$ & Scheme & 2nd Order Truncation Error \\
\hline-1 & Fully Upwind & $\frac{1}{3} \Delta \xi^{2}\left(\partial^{3} Q / \partial \xi^{3}\right)$ \\
0 & Fromm's & $\frac{1}{12} \Delta \xi^{2}\left(\partial^{3} Q / \partial \xi^{3}\right)$ \\
$1 / 3$ & Third Order & 0 \\
1 & Centered & $-\frac{1}{6} \Delta \xi^{2}\left(\partial^{3} Q / \partial \xi^{3}\right)$ \\
\hline
\end{tabular}

Table 1. Values of $\kappa$ and Associated Truncation Error ${ }^{[24]}$

The parameter $\kappa$ determines the accuracy of the spatial differencing. The common choices for $\kappa$ and their truncation errors are given in Table 1. For all values of $\kappa$ the scheme is second order accurate in space except for $\kappa=1 / 3$ when the scheme is third order accurate (strictly valid for a one dimensional problem). When higher-order differencing is used, the gradients $\delta Q$ have to be limited in regions of flow with discontinuities, such as shocks, to avoid oscillations of the flow variables. The choice of limiter is postponed until later.

If $E$ is a linear function of $Q$, i.e., $\partial E / \partial Q$ is a constant, then given the initial left and right states by Eq. 5-4, the exact solution of the Riemann problem can be written in terms of the flux difference as

$$
E_{R}-E_{L}=\mathbf{K}_{\xi}\left(Q_{R}-Q_{L}\right)
$$

where

$$
\mathbf{K}_{\xi}=\frac{\partial E}{\partial Q}
$$

if the flux Jacobian matrix. For the Euler equations it is possible to write the flux Jacobian matrix as $^{[27]}$

$$
\mathbf{K}_{\xi}=\mathbf{T}_{\xi} \Lambda_{\xi} \mathbf{T}_{\xi}^{-1}
$$

where columns of $\mathbf{T}_{\xi}$ are the right eigenvectors of $\mathbf{K}_{\xi}$, rows of $\mathbf{T}_{\xi}^{-1}$ are the left eigenvectors of $K_{\xi}$ and $\Lambda_{\xi}$ is a diagonal matrix containing the eigenvalues of $\mathbf{K}_{\xi}$. The evaluation of $\mathbf{T}_{\xi}, \mathbf{T}_{\xi}^{-1}$ and $\Lambda_{\xi}$ will be discussed later in the section. Substituting Eq. 5-9 in Eq. 5-7 we get

$$
E_{R}-E_{L}=\mathbf{T}_{\xi} \boldsymbol{\Lambda}_{\xi} \mathbf{T}_{\xi}^{-1}\left(Q_{R}-Q_{L}\right)
$$


Carrying out the matrix multiplication on the right-hand-side of Eq. 5-10, we can write

$$
E_{R}-E_{L}=\sum_{n=1}^{3} \alpha_{n} \lambda_{n} \vec{\varepsilon}_{n}
$$

where $\alpha_{n}$ is the projection of the difference between the initial left and right states on to the left eigenvectors of $\mathbf{K}_{\xi}$, i.e., $\alpha_{n}$ is the $n^{\text {th }}$ element of the vector $\mathbf{T}_{\xi}^{-1}\left(Q_{R}-Q_{L}\right), \lambda_{n}$ is the $n^{\text {th }}$ eigenvalue and $\vec{\varepsilon}_{n}$ the $n^{\text {th }}$ right eigenvector of $\mathbf{K}_{\xi}$. Each term of the summation represents the change in flux caused by the corresponding wave. $\alpha_{n}$ represents the strength of the $n^{\text {th }}$ wave, and $\lambda_{n}$ is the wave speed. This is shown schematically in Fig. 5-1. From Fig. 5-1, it is evident that the flux at the interface can be computed from either

$$
E_{i+\frac{1}{2}}\left(Q_{L}, Q_{R}\right)=E_{L}+\bar{\sum} \alpha_{n} \lambda_{n} \vec{\varepsilon}_{n}
$$

or

$$
E_{i+\frac{1}{2}}\left(Q_{L}, Q_{R}\right)=E_{R}-\sum^{+} \alpha_{n} \lambda_{n} \vec{\varepsilon}_{n}
$$

where $\bar{\sum}$ and $\sum^{+}$denote the summation of the change in flux due to negative and positive wave speeds respectively. Averiging Eq. 5-12 and Eq. 5-13 we get $^{[25]}$

$$
E_{i+\frac{1}{2}}\left(Q_{L}, Q_{R}\right)=\frac{1}{2}\left[E_{L}+E_{R}-\sum_{n=1}^{3} \alpha_{n}\left|\lambda_{n}\right| \vec{\varepsilon}_{n}\right]
$$

Eq. 5-14 can be written in the matrix notation as

$$
E_{i+\frac{1}{2}}\left(Q_{L}, Q_{R}\right)=\frac{1}{2}\left[E_{L}+E_{R}-\left|\mathbf{K}_{\xi}\right|\left(Q_{R}-Q_{L}\right)\right]
$$

where

$$
\left|\mathbf{K}_{\xi}\right|=\mathbf{T}_{\xi}\left|\Lambda_{\xi}\right| \mathbf{T}_{\xi}^{-1}
$$

For Euler equations, where $E$ is a nonlinear function of $Q$, we can define a locally constant matrix $K_{\xi}$ whose eigenvalues and eigenvectors satisfy Eq. 5-11 and also the condition

$$
Q_{R}-Q_{L}=\sum_{n=1}^{3} \alpha_{n} \vec{\varepsilon}_{n}
$$


For this method to return the exact solution whenever the left and right states lie on opposite sides of a shock wave or a contact discontinuity, the Rankine-Hugoniot condition,

$$
E_{R}-E_{L}=S\left(Q_{R}-Q_{L}\right)
$$

where $S$ is the speed of the discontinuity must be satisfied. $\mathrm{K}_{\xi}$, called the Roe-averaged matrix, can be written in the form

$$
K_{\xi}=\mathrm{T}_{\xi} \Pi_{\xi} \mathrm{T}_{\xi}^{-1}
$$

where $T_{\xi}$ is the right eigenvector matrix of $K_{\xi}, T_{\xi}^{-1}$ is the left eigenvector matrix of $K_{\xi}$ and $\Pi_{\xi}$ is a diagonal matrix containing the eigenvalues of $K_{\xi}$. Hence the flux difference in Eq. 5-15 can be written as

$$
E_{i+\frac{1}{2}}\left(Q_{L}, Q_{R}\right)=\frac{1}{2}\left[E_{L}+E_{R}-\left|\mathbf{K}_{\xi}\right|\left(Q_{R}-Q_{L}\right)\right]
$$

where

$$
\left|K_{\xi}\right|=\mathrm{T}_{\xi}\left|\Pi_{\xi}\right| \mathbf{T}_{\xi}^{-1}
$$

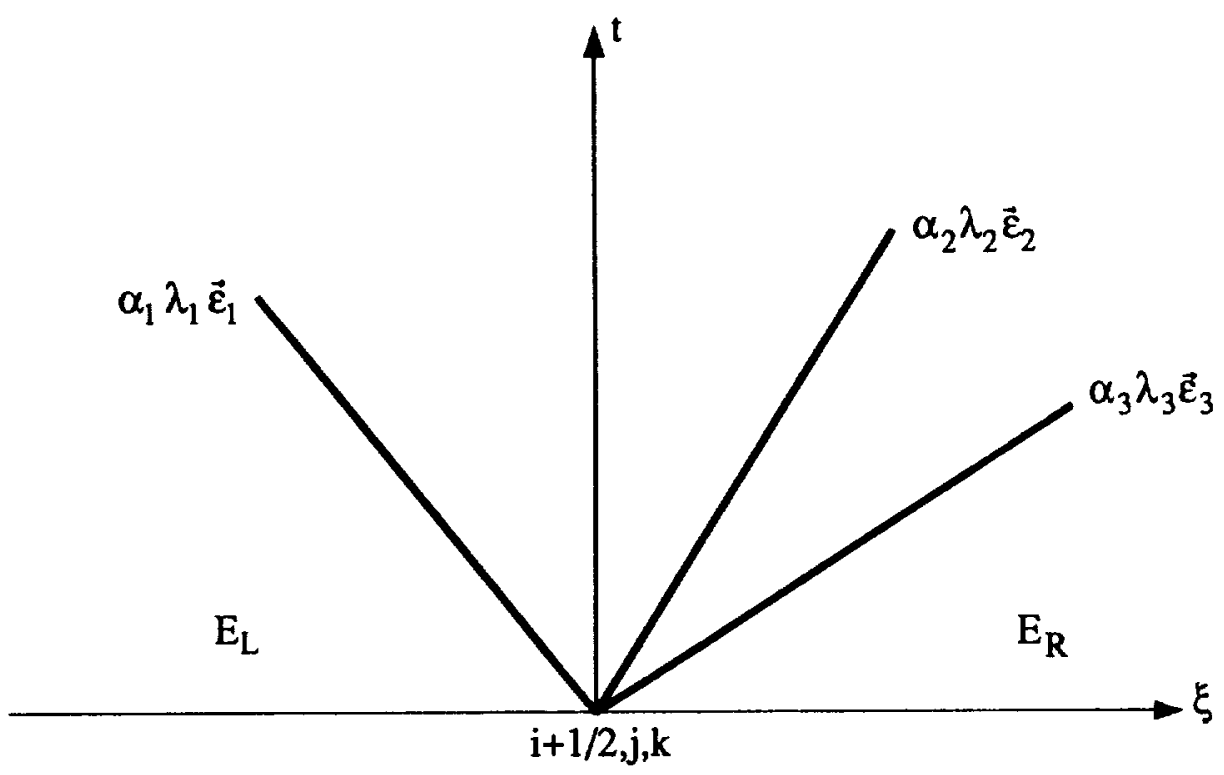

Figure 5-1. Schematic Representation of Waves at an Cell Interface 
The matrices $T_{\xi}, T_{\xi}^{-1}$ and $\Pi_{\xi}$ are obtained by replacing the quantities in $\mathbf{T}_{\xi}, \mathbf{T}_{\xi}^{-1}$ and $\Lambda_{\xi}$ by their Roe averages. The Roe averaged quantities are evaluated as follows ${ }^{[2]}$

$$
\begin{gathered}
\rho^{*}=\sqrt{\rho_{L} \rho_{R}} \\
f^{*}=\frac{\sqrt{\rho_{L}} f_{L}+\sqrt{\rho_{R}} f_{R}}{\sqrt{\rho_{L}}+\sqrt{\rho_{R}}}
\end{gathered}
$$

where

$$
f=(u, v, w, H)
$$

Again, the evaluation of $\mathbf{\Gamma}_{\xi}, \mathbf{T}_{\xi}^{-1}$ and $\boldsymbol{\Lambda}_{\xi}$ will be presented later in this section.

\section{Limiter}

There are several choices for the limiter ${ }^{[29]}$ to ensure that the Total Variation of the initial distribution of $Q$ is Diminishing (TVD). Here we choose the minmod limiter where $\delta Q_{i}^{+}$and $\delta Q_{i}^{-}$ are given by the following expressions:

$$
\begin{aligned}
& \delta Q_{i}^{+}=\operatorname{minmod}\left[\left(Q_{i+1}-Q_{i}\right), \beta\left(Q_{i}-Q_{i-1}\right)\right] \\
& \delta Q_{i}^{-}=\operatorname{minmod}\left[\left(Q_{i}-Q_{i-1}\right), \beta\left(Q_{i+1}-Q_{i}\right)\right]
\end{aligned}
$$

where

$$
\operatorname{minmod}(x, y)= \begin{cases}0 & \operatorname{sign}(x) \neq \operatorname{sign}(y) \\ \operatorname{sign}(x) \min (|x|,|y|) & \operatorname{sign}(x)=\operatorname{sign}(y)\end{cases}
$$

and

$$
\beta=\frac{3-\kappa}{1-\kappa}
$$

is a compression parameter. In smooth regions of the flow, the effect of the limiter is negligible. However, in the regions of the flow with high gradients, the accuracy reduces to first order. 


\section{Three-dimensional Problem}

The one-dimensional flux differencing technique can be extended to multidimensions by treating each direction in a locally one-dimensional manner. Although this approach allow interactions, of the flowfield, to occur only normal to the cell faces, it has been very successful in solving a variety of problems. For the three-dimensional problem the interface fluxes in each of the three directions are written as

$$
\begin{aligned}
& E_{i+\frac{1}{2}, j, k}=\frac{1}{2}\left[E_{L(i)}+E_{R(i)}-\left|K_{\xi}\right|\left(Q_{R(i)}-Q_{L(i)}\right)\right] \\
& F_{i, j+\frac{1}{2}, k}=\frac{1}{2}\left[F_{L(j)}+F_{R(j)}-\left|K_{\eta}\right|\left(Q_{R(j)}-Q_{L(j)}\right)\right] \\
& G_{i, j, k+\frac{1}{2}}=\frac{1}{2}\left[G_{L(k)}+G_{R(k)}-\left|K_{\zeta}\right|\left(Q_{R(k)}-Q_{L(k)}\right)\right]
\end{aligned}
$$

Here the flux-vectors have 5 elements and the flux Jacobian matrices are $5 \times 5$.

The contribution of the inviscid terms to the left hand side of Eq. 4-13 are evaluated as follows. The flux at the interface $i+\frac{1}{2}, j, k$ is

$$
E_{i+\frac{1}{2}, j, k}=\frac{1}{2}\left[E_{L}+E_{R}-\left|\mathrm{K}_{\xi}\right|\left(Q_{R}-Q_{L}\right)\right]
$$

Linearizing the flux at this cell-interface, we get

$$
\frac{\partial E_{i+\frac{1}{2}, j, k}}{\partial Q_{i+\frac{1}{2}, j, k}} \Delta Q_{i+\frac{1}{2}, j, k}=\frac{\partial E_{i+\frac{1}{2}, j, k}}{\partial Q_{L}} \Delta Q_{L}+\frac{\partial E_{i+\frac{1}{2}, j, k}}{\partial Q_{R}} \Delta Q_{R}
$$

Carrying out the differentiation we get

$$
\left(\frac{\partial E}{\partial Q} \Delta Q\right)_{i+\frac{1}{2}, j, k}=\frac{1}{2}\left[\frac{\partial E_{L}}{\partial Q_{L}} \Delta Q_{L}+\frac{\partial E_{R}}{\partial Q_{R}} \Delta Q_{R}-\left|\kappa_{\xi}\right|\left(\Delta Q_{R}-\Delta Q_{L}\right)-\frac{\partial\left|\kappa_{\xi}\right|}{\partial Q} \Delta Q\left(Q_{R}-Q_{L}\right)\right]
$$

Neglecting the higher-order term $\left(\partial\left|\mathrm{K}_{\xi}\right| / \partial Q\right) \Delta Q\left(Q_{R}-Q_{L}\right)$ we can write

$$
\left(\frac{\partial E}{\partial Q} \Delta Q\right)_{i+\frac{1}{2}, j, k} \approx \frac{1}{2}\left[\left(\frac{\partial E}{\partial Q} \Delta Q\right)_{L}+\left(\frac{\partial E}{\partial Q} \Delta Q\right)_{R}-\left|K_{\xi}\right|\left(\Delta Q_{R}-\Delta Q_{L}\right)\right]
$$

Making the approximation $(\partial E / \partial Q)_{L}=(\partial E / \partial Q)_{R}=K_{\xi}$ we can write

$$
\begin{aligned}
\left(\frac{\partial E}{\partial Q} \Delta Q\right)_{i+\frac{1}{2}, j, k} & \approx \frac{\mathrm{K}_{\xi}+\left|\mathrm{K}_{\xi}\right|}{2} \Delta Q_{L}+\frac{\mathrm{K}_{\xi}-\left|\mathrm{K}_{\xi}\right|}{2} \Delta Q_{R} \\
& =\left(\mathrm{T}_{\xi} \Pi_{\xi}^{+} \mathrm{T}_{\xi}^{-1}\right)_{i+\frac{1}{2}, j, k} \Delta Q_{L}+\left(\mathrm{T}_{\xi} \Pi_{\xi}^{-} \mathrm{T}_{\xi}^{-1}\right)_{i+\frac{1}{2}, j, k} \Delta Q_{R}
\end{aligned}
$$


where $\Pi_{\xi}^{+}$and $\Pi_{\xi}^{-}$are diagonal matrices containing the positive and negative eigenvalues respectively. Note that the matrices are evaluated at the cell interface using the Roe-averaged quantities. In order to obtain a tridiagonal system of equations, we choose $Q_{L}$ and $Q_{R}$ to be equal to $Q_{i, j, k}$ and $Q_{i+1, j, k}$ respectively, which is a first order approximation. Thus Eq. 5-33 becomes

$$
\left(\frac{\partial E}{\partial Q} \Delta Q\right)_{i+\frac{1}{2}, j, k} \approx\left(\mathrm{T}_{\xi} \Pi_{\xi}^{+} \mathrm{T}_{\xi}^{-1}\right)_{i+\frac{1}{2}, j, k} \Delta Q_{i, j, k}+\left(\mathrm{T}_{\xi} \Pi_{\xi}^{-} \mathrm{T}_{\xi}^{-1}\right)_{i+\frac{1}{2}, j, k} \Delta Q_{i+1, j, k}
$$

Similarly we can write

$$
\left(\frac{\partial E}{\partial Q} \Delta Q\right)_{i-\frac{1}{2}, j, k}==\left(\mathrm{T}_{\xi} \Pi_{\xi}^{+} \mathrm{T}_{\xi}^{-1}\right)_{i-\frac{1}{2}, j, k} \Delta Q_{i-1, j, k}+\left(\mathrm{T}_{\xi} \Pi_{\xi}^{-} \mathrm{T}_{\xi}^{-1}\right)_{i-\frac{1}{2}, j, k} \Delta Q_{i, j, k}
$$

\section{Eigenvalues and Eigenvectors}

Here we describe the evaluation of the eigenvalues and eigenvectors of the inviscid flux Jacobian matrices $\partial E / \partial Q, \partial F / \partial Q$ and $\partial G / \partial Q$. The inviscid fluxes $E, F$ and $G$ can be written in a general form as

$$
E, F \text { or } G=\frac{|\nabla r|}{J}\left[\begin{array}{c}
\rho \bar{u} \\
\rho H \bar{u} \\
\rho u \bar{u}+p \bar{r}_{x} \\
\rho v \bar{u}+p \bar{r}_{y} \\
\rho w \bar{u}+p \bar{r}_{z}
\end{array}\right] \text { for } r=\xi, \eta \text { or } \zeta
$$

where

$$
\bar{u}=u \bar{r}_{x}+v \bar{r}_{y}+w \bar{r}_{z}
$$

is the contravariant velocty component in the $r$-direction where $r$ stands for $\xi, \eta$ or $\zeta$. Let

$$
\begin{aligned}
& \mathbf{K}_{\xi}=\partial E / \partial Q \\
& \mathbf{K}_{\eta}=\partial F / \partial Q \\
& \mathbf{K}_{\zeta}=\partial G / \partial Q
\end{aligned}
$$

The inviscid flux Jacobiaus $\mathbf{K}_{\xi}, \mathbf{K}_{\eta}$ and $\mathbf{K}_{\zeta}$ can be written in a general form as

$$
\mathbf{K}_{r}=\frac{\nabla r \mid}{J} \mid\left[\begin{array}{ccccc}
0 & 0 & \multicolumn{1}{c}{\bar{r}_{x}} & \multicolumn{1}{c}{\bar{r}_{y}} & \multicolumn{1}{c}{\bar{r}_{z}} \\
\left(\sigma q^{2}-H\right) i \bar{c} & \gamma \bar{u} & H \bar{r}_{x}-\sigma u \bar{u} & H \bar{r}_{y}-\sigma v \bar{u} & H \bar{r}_{z}-\sigma w \bar{u} \\
\sigma q^{2} \bar{r}_{x}-u \bar{i} & \sigma \bar{r}_{x} & u \bar{r}_{x}-\sigma u \bar{r}_{x}+\bar{u} & u \bar{r}_{y}-\sigma v \bar{r}_{x} & u \bar{r}_{z}-\sigma w \bar{r}_{x} \\
\sigma q^{2} \bar{r}_{y}-v \bar{u} & \sigma \bar{r}_{y} & v \bar{r}_{x}-\sigma u \bar{r}_{y} & v \bar{r}_{y}-\sigma v \bar{r}_{y}+\bar{u} & v \bar{r}_{z}-\sigma w \bar{r}_{y} \\
\sigma q^{2} \bar{r}_{z}-w i & \sigma \bar{r}_{z} & w \bar{r}_{x}-\sigma u \bar{r}_{z} & w \bar{r}_{y}-\sigma v \bar{r}_{z} & w \bar{r}_{z}-\sigma w \bar{r}_{z}+\bar{u}
\end{array}\right]
$$


where

$$
\begin{gathered}
q^{2}=\frac{u^{2}+v^{2}+w^{2}}{2} \\
\sigma=\gamma-1
\end{gathered}
$$

and $r$ stands for $\xi, \eta$ or $\zeta$. It is difficult to determine the eigenvalues of $\mathbf{K}_{r}$. However by similarity transformation we can obtain a matrix $\mathbf{P}_{r}$ whose eigenvalues are easy to evaluate. We can write

$$
\mathbf{P}_{\mathbf{r}}=\mathbf{M}^{-1} \mathbf{K}_{\boldsymbol{r}} \mathbf{M}
$$

where

$$
\mathbf{M}=\frac{\partial Q}{\partial U}=\left[\begin{array}{ccccc}
1 & 0 & 0 & 0 & 0 \\
q^{2} & 1 / \sigma & \rho u & \rho v & \rho w \\
u & 0 & \rho & 0 & 0 \\
v & 0 & 0 & \rho & 0 \\
w & 0 & 0 & 0 & \rho
\end{array}\right]
$$

and the inverse

$$
\mathbf{M}^{-1}=\left(\frac{\partial Q}{\partial U}\right)^{-1}=\left[\begin{array}{ccccc}
1 & 0 & 0 & 0 & 0 \\
\sigma q^{2} & \sigma & -\sigma u & -\sigma v & -\sigma w \\
-u / \rho & 0 & 1 / \rho & 0 & 0 \\
-v / \rho & 0 & 0 & 1 / \rho & 0 \\
-w / \rho & 0 & 0 & 0 & 1 / \rho
\end{array}\right]
$$

and

$$
U=\left[\begin{array}{c}
\rho \\
p \\
u \\
v \\
w
\end{array}\right]
$$


Using Eqs. 5-39, 5-43 and 5-44 we get

$$
\mathbf{P}_{r}=\frac{|\nabla r|}{J}\left[\begin{array}{ccccc}
\bar{u} & 0 & \rho \bar{r}_{x} & \rho \bar{r}_{y} & \rho \bar{r}_{z} \\
0 & \bar{u} & \rho a^{2} \bar{r}_{x} & \rho a^{2} \bar{r}_{y} & \rho a^{2} \bar{r}_{z} \\
0 & \bar{r}_{x} / \rho & \bar{u} & 0 & 0 \\
0 & \bar{r}_{y} / \rho & 0 & \bar{u} & 0 \\
0 & \bar{r}_{z} / \rho & 0 & 0 & \bar{u}
\end{array}\right]
$$

Since matrices $K_{r}$ and $P_{r}$ are similar, their eigenvalues are the same ${ }^{[30]}$. Hence the eigenvalues of $\mathbf{K}_{r}$ can be found by determining the eigenvalues of $\mathbf{P}_{r}$. The eigenvalues of $\mathbf{P}_{r}$ are

$$
\left[\begin{array}{c}
\lambda_{r}^{+} \\
\lambda_{r}^{-} \\
\lambda_{r}^{0} \\
\lambda_{r}^{0} \\
\lambda_{r}^{0}
\end{array}\right]=\frac{|\nabla r|}{J}\left[\begin{array}{c}
\bar{u}+a \\
\bar{u}-a \\
\bar{u} \\
\bar{u} \\
\bar{u}
\end{array}\right]
$$

$\lambda_{r}^{+}$and $\lambda_{r}^{-}$are referred to as the acoustic eigenvalues and $\lambda_{r}^{0}$ is referred to as the convective eigenvalue.

Now we seek a matrix $\mathbf{S}_{r}$ that will diagonalize $\mathbf{P}_{r}$ such that

$$
\mathbf{S}_{r}^{-1} \mathbf{P}_{r} \mathbf{S}_{r}=\Lambda_{\mathbf{r}}
$$

where

$$
\boldsymbol{\Lambda}_{r}=\left[\begin{array}{ccccc}
\lambda_{r}^{+} & 0 & 0 & 0 & 0 \\
0 & \lambda_{r}^{-} & 0 & 0 & 0 \\
0 & 0 & \lambda_{r}^{0} & 0 & 0 \\
0 & 0 & 0 & \lambda_{r}^{0} & 0 \\
0 & 0 & 0 & 0 & \lambda_{r}^{0}
\end{array}\right]
$$

Columns of $\mathbf{S}_{r}$ are the right eigenvectors of $\mathbf{P}_{r}$ and the rows of $\mathbf{S}_{r}^{-1}$ are the left eigenvectors of $\mathbf{P}_{r}$. Skipping the algebra, an eigenvector matrix and its inverse that will diagonalize $\mathbf{P}_{r}$ as prescribed by Eq. $5-48$ are 


$$
\mathbf{S}_{r}=\left[\begin{array}{ccccc}
1 & 1 & 1 & 0 & 0 \\
a^{2} & a^{2} & 0 & 0 & 0 \\
a \bar{r}_{x} / \rho & -a \bar{r}_{x} / \rho & 0 & \bar{l}_{x} / \rho & \bar{m}_{x} / \rho \\
a \bar{r}_{y} / \rho & -a \bar{r}_{y} / \rho & 0 & \bar{l}_{y} / \rho & \bar{m}_{y} / \rho \\
a \bar{r}_{z} / \rho & -a \bar{r}_{z} / \rho & 0 & \bar{l}_{z} / \rho & \bar{m}_{z} / \rho
\end{array}\right]
$$

and

$$
\mathbf{S}_{r}^{-1}=\left[\begin{array}{ccccc}
0 & 1 / 2 a^{2} & \rho \bar{r}_{x} / 2 a & \rho \bar{r}_{y} / 2 a & \rho \bar{r}_{z} / 2 a \\
0 & 1 / 2 a^{2} & -\rho \bar{r}_{x} / 2 a & -\rho \bar{r}_{y} / 2 a & -\rho \bar{r}_{z} / 2 a \\
1 & -1 / a^{2} & 0 & 0 & 0 \\
0 & 0 & \rho \bar{l}_{x} & \rho \bar{l}_{y} & \rho \bar{l}_{z} \\
0 & 0 & \rho \bar{m}_{x} & \rho \bar{m}_{y} & \rho \bar{m}_{z}
\end{array}\right]
$$

where $\bar{l}_{x}, \bar{l}_{y}, \bar{l}_{z}$ and $\bar{m}_{x}, \bar{m}_{y}, \bar{m}_{z}$ are the components of two, arbitrary, mutually perpendicular unit vectors $\bar{l}$ and $\bar{m}$ respectively that are orthogonal to $\bar{r}$ whose components are $\bar{r}_{x}, \bar{r}_{y}, \bar{r}_{z}$.

Using Eqs. 5-42 and 5-48 we can write

$$
\mathbf{K}_{r}=\mathbf{T}_{r} \Lambda_{r} \mathbf{T}_{r}^{-1}
$$

where

$$
\mathbf{T}_{r}=\mathbf{M} \mathbf{S}_{r}=\left[\begin{array}{ccccc}
1 & 1 & 1 & 0 & 0 \\
H+a \bar{u} & H-a \bar{u} & q^{2} & \bar{v} & \bar{w} \\
u+a \bar{r}_{x} & u-a \bar{r}_{x} & u & \bar{l}_{x} & \bar{m}_{x} \\
v+a \bar{r}_{y} & v-a \bar{r}_{y} & v & \bar{l}_{y} & \bar{m}_{y} \\
w+a \bar{r}_{z} & w-a \bar{r}_{z} & w & \bar{l}_{z} & \bar{m}_{z}
\end{array}\right]
$$

and

$$
\mathbf{T}_{r}^{-1}=\mathbf{S}_{r}^{-1} \mathbf{M}_{r}^{-1}=\frac{1}{2 a^{2}}\left[\begin{array}{ccccc}
\sigma q^{2}-a \bar{u} & \sigma & a \bar{r}_{x}-\sigma u & a \bar{r}_{y}-\sigma v & a \bar{r}_{z}-\sigma w \\
\sigma q^{2}+a \bar{u} & \sigma & -a \bar{r}_{x}-\sigma u & -a \bar{r}_{y}-\sigma v & -a \bar{r}_{z}-\sigma w \\
2 a^{2}-2 \sigma q^{2} & -2 \sigma & 2 \sigma u & 2 \sigma v & 2 \sigma w \\
-2 a^{2} \bar{v} & 0 & 2 a^{2} \bar{l}_{x} & 2 a^{2} \bar{l}_{y} & 2 a^{2} \bar{l}_{z} \\
-2 a^{2} \bar{w} & 0 & 2 a^{2} \bar{m}_{x} & 2 a^{2} \bar{m}_{y} & 2 a^{2} \bar{m}_{z}
\end{array}\right]
$$

where

$$
\bar{v}=u \bar{l}_{x}+v \bar{l}_{y}+w \bar{l}_{z}
$$

and

$$
\bar{w}=u \bar{m}_{x}+v \bar{m}_{y}+w \bar{m}_{z}
$$


are two mutually perpendicular velocity components that are orthogonal to $\bar{u}$. Substituting Eqs. 549, 5-53 and 5-54 in Eq. 5-52, we can obtain the matrix $K_{r}$. Matrix $\left|K_{r}\right|$ can be obtained from $K_{r}$ by replacing all the flow quantities by their Roe averages given by Eqs. 5-22, 5-23 and 5-24, and replacing the eigenvalues by their magnitude. The flux differences $\left|K_{r}\right|\left(Q_{R}-Q_{L}\right)$ in Eq. 5-28 can be written in a computationally efficient form as

$$
\begin{aligned}
& \left|K_{r}\right|\left(Q_{R}-Q_{L}\right)=\frac{|\nabla r|}{J}\left|\bar{u}^{*}+a^{*}\right|\left[\begin{array}{c}
1 \\
H^{*}+a^{*} \bar{u}^{*} \\
u^{*}+\bar{r}_{x} a^{*} \\
v^{*}+\bar{r}_{y} a^{*} \\
w^{*}+\bar{r}_{z} a^{*}
\end{array}\right]\left\{\frac{p_{R}-p_{L}}{2\left(a^{*}\right)^{2}}+\frac{\rho^{*}\left(\bar{u}_{R}-\bar{u}_{L}\right)}{2 a^{*}}\right\} \\
& +\frac{|\nabla r|}{J}\left|\bar{u}^{*}-a^{*}\right|\left[\begin{array}{c}
1 \\
H^{*}-a^{*} \bar{u}^{*} \\
u^{*}-\bar{r}_{x} a^{*} \\
v^{*}-\bar{r}_{y} a^{*} \\
w^{*}-\bar{r}_{z} a^{*}
\end{array}\right]\left\{\frac{p_{R}-p_{L}}{2\left(a^{*}\right)^{2}}-\frac{\rho^{*}\left(\bar{u}_{R}-\bar{u}_{L}\right)}{2 a^{*}}\right\} \\
& +\frac{|\nabla r|}{J}\left|\bar{u}^{*}\right|\left[\begin{array}{c}
1 \\
\left(q^{*}\right)^{2} \\
u^{*} \\
v^{*} \\
w^{*}
\end{array}\right]\left\{\rho_{R}-\rho_{L}-\frac{p_{R}-p_{L}}{\left(a^{*}\right)^{2}}\right\} \\
& +\frac{|\nabla r|}{J}\left|\bar{u}^{*}\right| \rho^{*}\left[\begin{array}{c}
0 \\
u^{*}\left(\iota_{R}-u_{L}\right)+v^{*}\left(v_{R}-v_{L}\right)+w^{*}\left(w_{R}-w_{L}\right)-\bar{u}^{*}\left(\bar{u}_{R}-\bar{u}_{L}\right) \\
u_{R}-u_{L}-\bar{r}_{x}\left(\bar{u}_{R}-\bar{u}_{L}\right) \\
v_{R}-v_{L}-\bar{r}_{y}\left(\bar{u}_{R}-\bar{u}_{L}\right) \\
w_{R}-w_{L}-\bar{r}_{z}\left(\bar{u}_{R}-\bar{u}_{L}\right)
\end{array}\right]
\end{aligned}
$$

where the quantities with a star are Roe-averaged quantities. Using Eq. 5-57 in Eq. 5-28 the contribution to the right hand side of Eq. 4-13, due to the inviscid terms can be evaluated. In order to determine the left hand side, we have to evaluate the matrices $\mathrm{T}_{r} \Pi_{r}^{+} \mathrm{T}_{r}^{-1}$ and $\mathrm{T}_{r} \Pi_{r}^{-} \mathrm{T}_{r}^{-1}$. The evaluation the matrices $T_{r} \Pi_{r}^{+} T_{r}^{-1}$ and $T_{r} \Pi_{r}^{-} T_{r}^{-1}$ is given in Appendix $B$. 


\section{Discretization of the Viscous Fluxes}

In this chapter we discuss the treatment of the viscous terms. The viscous fluxes $\mathcal{E}, \mathcal{F}$ and $\mathcal{G}$ can be written in a general form as

$$
\mathcal{E}, \mathcal{F} \text { or } \mathcal{G}=\frac{|\nabla r|}{J}\left[\begin{array}{c}
0 \\
u \tau_{r \xi}+v \tau_{r \eta}+w \tau_{r \zeta}-\dot{q_{r}} \\
\tau_{r \xi} \\
\tau_{r \eta} \\
\tau_{r \zeta}
\end{array}\right] \text { for } r=\xi, \eta \text { or } \zeta
$$

where

$$
\begin{aligned}
& \tau_{r \xi}=\tau_{x x} \bar{r}_{x}+\tau_{y x} \bar{r}_{y}+\tau_{z x} \bar{r}_{z} \\
& \tau_{r \eta}=\tau_{x y} \bar{r}_{x}+\tau_{y y} \bar{r}_{y}+\tau_{z y} \bar{r}_{z} \\
& \tau_{r \zeta}=\tau_{x z} \bar{r}_{x}+\tau_{y z} \bar{r}_{y}+\tau_{z z} \bar{r}_{z}
\end{aligned}
$$

and

$$
\dot{q}_{\mathrm{r}}=\dot{q}_{x} \bar{r}_{x}+\dot{q}_{y} \bar{r}_{y}+\dot{q}_{z} \bar{r}_{z}
$$

where $r$ stands for $\xi, \eta$ or $\zeta$. Note that the shear-stress and heat-transfer terms contain spatial derivatives with respect to $x, y$ and $z$. They can be evaluated in the transformed space, $(\xi, \eta, \zeta)$, using the chainrule and hence we can write

$$
\left[\begin{array}{llll}
u_{x} & v_{x} & w_{x} & T_{x} \\
u_{y} & v_{y} & w_{y} & T_{y} \\
u_{z} & v_{z} & w_{z} & T_{z}
\end{array}\right]=\left[\begin{array}{lll}
\xi_{x} & \eta_{x} & \zeta_{x} \\
\xi_{y} & \eta_{y} & \zeta_{y} \\
\xi_{z} & \eta_{z} & \zeta_{z}
\end{array}\right]\left[\begin{array}{llll}
u_{\xi} & v_{\xi} & w_{\xi} & T_{\xi} \\
u_{\eta} & v_{\eta} & w_{\eta} & T_{\eta} \\
u_{\zeta} & v_{\zeta} & w_{\zeta} & T_{\zeta}
\end{array}\right]
$$

Substituting for the shear-stress and heat-transfer terms in Eq. 6-1 from Eqs. 2-7 through 2-15 and using Eq. 6-4 we can write the viscous fluxes as

$$
\mathcal{E}, \mathcal{F} \text { or } \mathcal{G}=\frac{|\nabla r|}{J} \frac{M_{\infty}}{R e} \mu\left[\begin{array}{c}
0 \\
\Phi_{2 r} \\
\Phi_{3 r} \\
\Phi_{4 r} \\
\Phi_{5 r}
\end{array}\right] \text { for } r=\xi, \eta \text { or } \zeta
$$

where

$$
\begin{aligned}
& \Phi_{3 r}=\phi_{1 \xi r} u_{\xi}+\phi_{2 \xi r} v_{\xi}+\phi_{3 \xi r} w_{\xi}+\phi_{1 \eta r} u_{\eta}+\phi_{2 \eta r} v_{\eta}+\phi_{3 \eta r} w_{\eta}+\phi_{1 \zeta r} u_{\zeta}+\phi_{2 \zeta r} v_{\zeta}+\phi_{3 \zeta} w_{\zeta} \\
& \Phi_{4 r}=\phi_{4 \xi r} u_{\xi}+\phi_{5 \xi r} v_{\xi}+\phi_{6 \xi r} w_{\xi}+\phi_{4 \eta r} u_{\eta}+\phi_{5 \eta r} v_{\eta}+\phi_{6 \eta r} w_{\eta}+\phi_{4 \zeta r} u_{\zeta}+\phi_{5 \zeta r} v_{\zeta}+\phi_{6 \zeta r} w_{\zeta} \\
& \Phi_{5 r}=\phi_{7 \xi r} u_{\xi}+\phi_{8 \xi r} v_{\xi}+\phi_{9 \xi r} w_{\xi}+\phi_{7 \eta r} u_{\eta}+\phi_{8 \eta r} v_{\eta}+\phi_{9 \eta r} w_{\eta}+\phi_{7 \zeta r} u_{\zeta}+\phi_{8 \zeta r} v_{\zeta}+\phi_{9 \zeta r} w_{\zeta} \\
& \Phi_{2 r}=\Phi_{3 r} u+\Phi_{4 r} v+\Phi_{5 r} w+\frac{\gamma}{\sigma P_{r}}\left(\phi_{0 \xi r} T_{\xi}+\phi_{0 \eta r} T_{\eta}+\phi_{0 \zeta r} T_{\zeta}\right)
\end{aligned}
$$


where

$$
\begin{aligned}
& \phi_{0 \xi \tau}=\xi_{x} \bar{r}_{x}+\xi_{y} \bar{r}_{y}+\xi_{z} \bar{r}_{z} \\
& \phi_{0 \eta r}=\eta_{x} \bar{r}_{x}+\eta_{y} \bar{r}_{y}+\eta_{z} \bar{r}_{z} \\
& \phi_{0 \zeta r}=\zeta_{x} \bar{r}_{x}+\zeta_{y} \bar{r}_{y}+\zeta_{z} \bar{r}_{z} \\
& \phi_{1 \xi r}=\phi_{0 \xi r}+\frac{1}{3} \xi_{x} \bar{r}_{x} \\
& \phi_{2 \xi r}=\xi_{x} \bar{r}_{y}-\frac{2}{3} \xi_{y} \bar{r}_{x} \\
& \phi_{3 \xi r}=\xi_{x} \bar{r}_{z}-\frac{2}{3} \xi_{z} \bar{r}_{x} \\
& \phi_{1 \eta r}=\phi_{0 \eta r}+\frac{1}{3} \eta_{x} \bar{r}_{x} \\
& \phi_{2 \eta r}=\eta_{x} \bar{r}_{y}-\frac{2}{3} \eta_{y} \bar{r}_{x} \\
& \phi_{3 \eta r}=\eta_{x} \bar{r}_{z}-\frac{2}{3} \eta_{z} \bar{r}_{x} \\
& \phi_{1 \zeta r}=\phi_{0 \zeta \tau}+\frac{1}{3} \zeta_{x} \bar{r}_{x} \\
& \phi_{2 \zeta r}=\zeta_{x} \vec{r}_{y}-\frac{2}{3} \zeta_{y} \bar{r}_{x} \\
& \phi_{3 \zeta r}=\zeta_{x} \bar{r}_{z}-\frac{2}{3} \zeta_{z} \bar{r}_{x} \\
& \phi_{4 \xi r}=\xi_{y} \bar{r}_{x}-\frac{2}{3} \xi_{x} \bar{r}_{y} \\
& \phi_{5 \xi r}=\phi_{0 \xi r}+\frac{1}{3} \xi_{y} \bar{r}_{y} \\
& \phi_{B \xi r}=\xi_{y} \bar{r}_{z}-\frac{2}{3} \xi_{z} \bar{r}_{y} \\
& \phi_{4 \eta \boldsymbol{r}}=\eta_{y} \bar{r}_{x}-\frac{2}{3} \eta_{x} \bar{r}_{y} \\
& \phi_{5 \eta r}=\phi_{0 \eta r}+\frac{1}{3} \eta_{y} \bar{r}_{y} \\
& \phi_{6 \eta r}=\eta_{y} \bar{r}_{z}-\frac{2}{3} \eta_{z} \bar{r}_{y} \\
& \phi_{4 \zeta r}=\zeta_{y} \bar{r}_{x}-\frac{2}{3} \zeta_{x} \bar{r}_{y} \\
& \phi_{5 \zeta r}=\phi_{0 \zeta r}+\frac{1}{3} \zeta_{y} \bar{r}_{y} \\
& \phi_{6 \zeta r}=\zeta_{y} \bar{r}_{z}-\frac{2}{3} \zeta_{z} \bar{r}_{y}
\end{aligned}
$$




$$
\begin{aligned}
& \phi_{7 \xi r}=\xi_{z} \bar{r}_{x}-\frac{2}{3} \xi_{x} \bar{r}_{z} \\
& \phi_{8 \xi r}=\xi_{z} \bar{r}_{y}-\frac{2}{3} \xi_{y} \bar{r}_{z} \\
& \phi_{9 \xi r}=\phi_{0 \xi r}+\frac{1}{3} \xi_{z} \bar{r}_{z} \\
& \phi_{7 \eta r}=\eta_{z} \bar{r}_{x}-\frac{2}{3} \eta_{x} \bar{r}_{z} \\
& \phi_{8 \eta r}=\eta_{z} \bar{r}_{y}-\frac{2}{3} \eta_{y} \bar{r}_{z} \\
& \phi_{9 \eta r}=\phi_{0 \eta r}+\frac{1}{3} \eta_{z} \bar{r}_{z} \\
& \phi_{7 \zeta r}=\zeta_{z} \bar{r}_{x}-\frac{2}{3} \zeta_{x} \bar{r}_{z} \\
& \phi_{8 \zeta r}=\zeta_{z} \bar{r}_{y}-\frac{2}{3} \zeta_{y} \bar{r}_{z} \\
& \phi_{9 \zeta r}=\phi_{0 \zeta r}+\frac{1}{3} \zeta_{z} \bar{r}_{z}
\end{aligned}
$$

where $r$ stands for $\xi, \eta$ or $\zeta$.

Once the metrics Eq. 2-26 are determined at each cell-interface (Evaluation of metrics will be discussed in the next section.), the quantities given by Eqs. 6-7, 6-8, 6-9 and 6-10 can be determined and hence the viscous fluxes through the cell-interfaces can be computed from Eqs. 65 and 6-6. For example,

$$
\mathcal{E}_{i+\frac{1}{2}, j, k}=\left(\frac{|\nabla \xi|}{J}\right)_{i+\frac{1}{2}, j, k} \frac{M_{\infty}}{R e} \mu_{i+\frac{1}{2}, j, k}\left[\begin{array}{c}
0 \\
\Phi_{2 \xi} \\
\Phi_{3 \xi} \\
\Phi_{4 \xi} \\
\Phi_{5 \xi}
\end{array}\right]_{i+\frac{1}{2}, j, k}
$$

where

$$
\begin{aligned}
& \Phi_{3 \xi}=\phi_{1 \xi \xi} u_{\xi}+\phi_{2 \xi \xi} v_{\xi}+\phi_{3 \xi \xi} w_{\xi}+\phi_{1 \eta \xi} u_{\eta}+\phi_{2 \eta \xi} v_{\eta}+\phi_{3 \eta \xi} w_{\eta}+\phi_{1 \zeta \xi} u_{\zeta}+\phi_{2 \zeta \xi} v_{\zeta}+\phi_{3 \zeta \xi} w_{\zeta} \\
& \boldsymbol{\Phi}_{4 \xi}=\phi_{4 \xi \xi} u_{\xi}+\phi_{5 \xi \xi} v_{\xi}+\phi_{6 \xi \xi} w_{\xi}+\phi_{4 \eta \xi} u_{\eta}+\phi_{5 \eta \xi} v_{\eta}+\phi_{6 \eta \xi} w_{\eta}+\phi_{4 \zeta \xi} u_{\zeta}+\phi_{5 \zeta \xi} v_{\zeta}+\phi_{B \zeta \xi} w_{\zeta} \\
& \Phi_{5 \xi}=\phi_{7 \xi \xi} u_{\xi}+\phi_{8 \xi \xi} v_{\xi}+\phi_{9 \xi \xi} w_{\xi}+\phi_{7 \eta \xi} u_{\eta}+\phi_{8 \eta \xi} v_{\eta}+\phi_{9 \eta \xi} w_{\eta}+\phi_{7 \zeta \xi} u_{\zeta}+\phi_{8 \zeta \xi} v_{\zeta}+\phi_{9 \zeta \xi} w_{\zeta} \\
& \Phi_{2 \xi}=\Phi_{3 \xi} u+\Phi_{4 \xi} v+\Phi_{5 \xi} w+\frac{\gamma}{\sigma P_{r}}\left(\phi_{0 \xi \xi} T_{\xi}+\phi_{0 \eta \xi} T_{\eta}+\phi_{0 \zeta \xi} T_{\zeta}\right)
\end{aligned}
$$

where 


$$
\begin{gathered}
{\left[\begin{array}{c}
u_{\xi} \\
v_{\xi} \\
w_{\xi} \\
T_{\xi}
\end{array}\right]_{i+\frac{1}{2}, j, k}=\left(f_{\xi}\right)_{i+\frac{1}{2}, j, k}=f_{i+1, j, k}-f_{i, j, k}} \\
{\left[\begin{array}{c}
u_{\eta} \\
v_{\eta} \\
w_{\eta} \\
T_{\eta}
\end{array}\right]_{i+\frac{1}{2}, j, k}=\left(f_{\eta}\right)_{i+\frac{1}{2}, j, k}=\frac{1}{4}\left(f_{i+1, j+1, k}+f_{i, j+1, k}-f_{i+1, j-1, k}-f_{i, j-1, k}\right)} \\
{\left[\begin{array}{c}
u_{\zeta} \\
v_{\zeta} \\
w_{\zeta} \\
T_{\zeta}
\end{array}\right]_{i+\frac{1}{2}, j, k}=\left(f_{\zeta}\right)_{i+\frac{1}{2}, j, k}=\frac{1}{4}\left(f_{i+1, j, k+1}+f_{i, j, k+1}-f_{i+1, j, k-1}-f_{i, j, k-1}\right)}
\end{gathered}
$$

and

$$
\left[\begin{array}{c}
u \\
v \\
w \\
\mu
\end{array}\right]_{i+\frac{1}{2}, j, k}=f_{i+\frac{1}{2}, j, k}=\frac{1}{2}\left(f_{i+1, j, k}+f_{i, j, k}\right)
$$

Notice that the cross-derivative terms are evaluated using a symmetric difference molecule and hence there is no contribution from these terms to the diagonal element of the coefficient matrix. Similarly the viscous fluxes at the other cell-interfaces can be determined. From the viscous fluxes at the cell-interface, the ccntribution of the viscous terms to the right hand side of Eq. 4-13 can be determined.

On the left hand side we have to evaluate the flux Jacobian matrices $\partial \mathcal{E} / \partial Q, \partial \mathcal{F} / \partial Q$ and $\partial \mathcal{G} / \partial Q$ of the viscous fluxes $\mathcal{E}, \mathcal{F}$ and $\mathcal{G}$. In order to obtain a tridiagonal system of equations, we neglect the cross-derivatives before the fluxes are linearized. For example, consider the fluxvector $\mathcal{E}$. First we neglect all the derivatives with respect to $\eta$ and $\zeta$. Then we discretize the terms according to Eqs. 6-13 and 6-16 and linearize them in the following manner. We can write,

$$
\left(\frac{\partial \mathcal{E}}{\partial Q} \Delta Q\right)_{i+\frac{1}{2}, j, k}=\frac{\partial \mathcal{E}_{i+\frac{1}{2}, j, k}}{\partial Q_{i+1, j, k}} \Delta Q_{i+1, j, k}+\frac{\partial \mathcal{E}_{i+\frac{1}{2}, j, k}}{\partial Q_{i, j, k}} \Delta Q_{i, j, k}
$$

Assuming that the molecular viscosity $\mu$ is locally constant and carrying out the differentiation and simplifying we can write

$$
\left(\frac{\partial \mathcal{E}}{\partial Q} \Delta Q\right)_{i+\frac{1}{2}, j, k}=\left(\mathrm{v}_{\xi}^{+}\right)_{i+\frac{1}{2}, j, k} \Delta Q_{i+1, j, k}-\left(\mathrm{v}_{\xi}^{-}\right)_{i+\frac{1}{2}, j, k} \Delta Q_{i, j, k}
$$


where

$$
\left(\mathbf{V}_{\xi}^{+}\right)_{i+\frac{1}{2}, j, k}=\left(\frac{\partial \mathcal{E}}{\partial Q}\right)_{i+1, j, k}
$$

and

$$
\left(\mathbf{V}_{\xi}^{-}\right)_{i+\frac{1}{2}, j, k}=-\left(\frac{\partial \mathcal{E}}{\partial Q}\right)_{i, j, k}
$$

The viscous flux Jacobian matrices $\left(\mathbf{V}_{\xi}^{+}\right)_{i+\frac{1}{2}, j, k}$ and $\left(\mathbf{V}_{\xi}^{-}\right)_{i+\frac{1}{2}, j, k}$ are functionally the same. The flow variables in $\left(\mathrm{V}_{\xi}^{+}\right)_{i+\frac{1}{2}, j, k}$ except $\mu$ are evaluated at the $(i+1, j, k)^{\text {th }}$ cell-center and the flow variables in $\left(\mathrm{V}_{\xi}^{-}\right)_{i+\frac{1}{2}, j, k}$ except $\mu$ are evaluated at the $(i, j, k)^{t h}$ cell-center. However the metrics are evaluated at the $\left(i+\frac{1}{2}, j, k\right)^{\text {th }}$ cell-interface. $\mu$ is evaluated at the cell-interface as an average given by Eq. 6-16 Similarly,

$$
\left(\frac{\partial \mathcal{E}}{\partial Q} \Delta Q\right)_{i-\frac{1}{2}, j, k}=\left(\mathbf{V}_{\xi}^{+}\right)_{i-\frac{1}{2}, j, k} \Delta Q_{i, j, k}-\left(\mathbf{V}_{\xi}^{-}\right)_{i-\frac{1}{2}, j, k} \Delta Q_{i-1, j, k}
$$

where

$$
\left(\mathrm{V}_{\xi}^{+}\right)_{i-\frac{1}{2}, j, k}=\left(\frac{\partial \mathcal{E}}{\partial Q}\right)_{i, j, k}
$$

and

$$
\left(\mathrm{v}_{\bar{\xi}}^{-}\right)_{i-\frac{1}{2}, j, k}=-\left(\frac{\partial \mathcal{E}}{\partial Q}\right)_{i-1, j, k}
$$

The other viscous flux Jacobian matrices $\partial \mathcal{F} / \partial Q$ and $\partial \mathcal{G} / \partial Q$ can be evaluated in a similar manner. Let $V_{r}$ denote the general viscous flux Jacobian matrix where, $r$ stands for $\xi, \eta$ and $\zeta$ such that

$$
\begin{aligned}
\mathbf{V}_{\xi} & =\partial \mathcal{E} / \partial Q \\
\mathbf{V}_{\eta} & =\partial \mathcal{F} / \partial Q \\
\mathbf{V}_{\zeta} & =\partial \mathcal{G} / \partial Q
\end{aligned}
$$

$V_{r}$ can be written in a general form as 


$$
\begin{aligned}
& \mathbf{V}_{r}=J\left(\frac{|\nabla r|}{J}\right)^{2} \frac{M_{\infty}}{R e} \xi^{\prime} \times \\
& {\left[\begin{array}{ccccc}
0 & 0 & 0 & 0 & 0 \\
-2 q^{2}-3 \widehat{u}^{2}-\gamma\left(e-2 q^{2}\right) / P r & \gamma / P r & u+\bar{r}_{x} \widehat{u}-\gamma u / P r & v+\bar{r}_{y} \widehat{u}-\gamma v / P r & w+\bar{r}_{z} \widehat{u}-\gamma w / P r \\
-u-\bar{r}_{x} \widehat{u} & 0 & 1+\bar{r}_{x} \bar{r}_{x} / 3 & \bar{r}_{x} \bar{r}_{y} / 3 & \bar{r}_{x} \bar{r}_{z} / 3 \\
-v-\bar{r}_{y} \widehat{u} & 0 & \bar{r}_{y} \bar{r}_{x} / 3 & 1+\bar{r}_{y} \bar{r}_{y} / 3 & \bar{r}_{y} \bar{r}_{z} / 3 \\
-w-\bar{r}_{z} \widehat{u} & 0 & \bar{r}_{z} \bar{r}_{x} / 3 & \bar{r}_{z} \bar{r}_{y} / 3 & 1+\bar{r}_{z} \bar{r}_{z} / 3
\end{array}\right]}
\end{aligned}
$$

where

$$
\widehat{u}=\frac{1}{3}\left(u \bar{r}_{x}+v \bar{r}_{y}+w \bar{r}_{z}\right)
$$

Again all the flow variables except $\mu$ are evaluated at the cell-center. $\mu$ is evaluated as the average of the adjacent cell values (Eq. 6-16). All the metrics, area and volume, $1 / J$, are evaluated at the cell-interface. The volume at the cell-interface is evaluated as the average of the volume of the adjacent cells. Some of the details of the derivation of $V_{r}$ are given in Appendix $C$. 


\section{Metrics, Areas and Volume}

From the numerical grid generation scheme, we get (Eq. 3-1),

$$
\begin{aligned}
& x=x\left(i \pm \frac{1}{2}, j \pm \frac{1}{2}, k \pm \frac{1}{2}\right) \\
& y=y\left(i \pm \frac{1}{2}, j \pm \frac{1}{2}, k \pm \frac{1}{2}\right) \\
& z=z\left(i \pm \frac{1}{2}, j \pm \frac{1}{2}, k \pm \frac{1}{2}\right)
\end{aligned}
$$

Eq. 7-1 describes a set a of hexahedrons in the flowfield. Note that in general, a grid in an arbitrary space may not yield hexahedrons, i.e., four adjacent points may not be coplanar.

Let ABCDEFGH shown in Fig. 7-1 represent a cell $(i, j, k)$ in the flowfield. The cell average of the flow variables, $Q$ are located at the cell center $(i, j, k)$. The vertices of the cell are

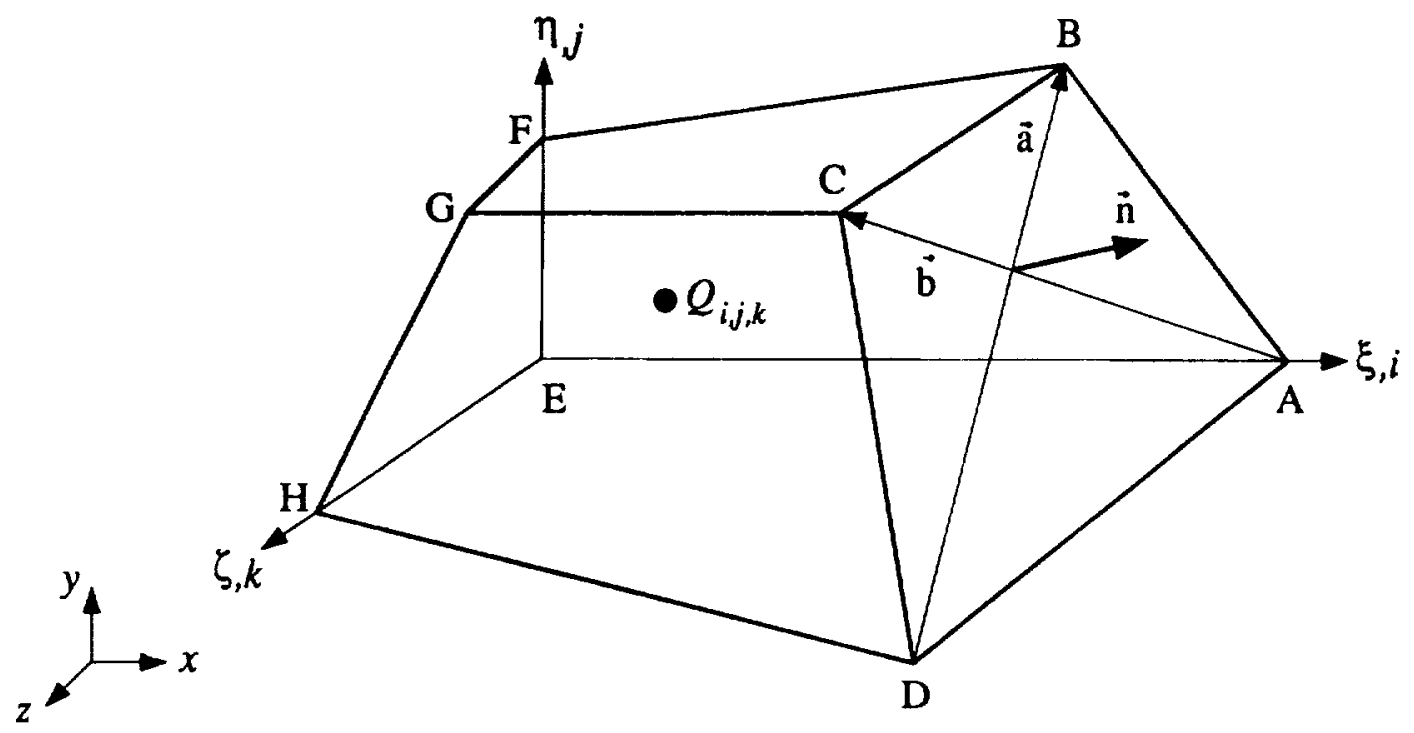

Figure 7-1. A Cell 

$\mathrm{A}:\left(i+\frac{1}{2}, j-\frac{1}{2}, k-\frac{1}{2}\right)$
$\mathrm{B}:\left(i+\frac{1}{2}, j+\frac{1}{2}, k-\frac{1}{2}\right)$
$\mathrm{C}:\left(i+\frac{1}{2}, j+\frac{1}{2}, k+\frac{1}{2}\right)$
$\mathrm{D}:\left(i+\frac{1}{2}, j-\frac{1}{2}, k+\frac{1}{2}\right)$
$\mathrm{E}:\left(i-\frac{1}{2}, j-\frac{1}{2}, k-\frac{1}{2}\right)$
$\mathrm{F}:\left(i-\frac{1}{2}, j+\frac{1}{2}, k-\frac{1}{2}\right)$
$\mathrm{G}:\left(i-\frac{1}{2}, j+\frac{1}{2}, k+\frac{1}{2}\right)$
$\mathrm{H}:\left(i-\frac{1}{2}, j-\frac{1}{2}, k+\frac{1}{2}\right)$

We need to evaluate the metrics, cell-face areas, cell-face normals and volume, of each of these cells, in order to solve the governing equations. Consider the constant $\xi$ face ABCD. Let $\vec{a}$ and $\vec{b}$ be the two diagonal vectors on the face. Then it can be shown that

$$
\frac{\xi_{x}}{J} \vec{i}+\frac{\xi_{y}}{J} \vec{j}+\frac{\xi_{z}}{J} \vec{k}=\frac{1}{2} \vec{a} \times \vec{b}
$$

is the directed face area and the resulting vector is perpendicular to the face. Therefore the unitnormal to the face is

$$
\vec{n}=\bar{\xi}_{x} \vec{i}+\bar{\xi}_{y} \vec{j}+\bar{\xi}_{z} \vec{k}=\frac{\vec{a} \times \vec{b}}{|\vec{a} \times \vec{b}|}
$$

The magnitude of the facis area is

$$
\frac{|\nabla \xi|}{J}=\frac{1}{2}|\vec{a} \times \vec{b}|
$$

Similar evaluations can be done for the other faces. To evaluate the inviscid fluxes on a constant $\xi$ face, the metrics associated with the other directions namely, $\eta_{x}, \eta_{y}, \eta_{z}, \zeta_{x}, \zeta_{y}$ and $\zeta_{z}$ are not need at the face. Similarly for the other directions. However all the metric quantities are needed at any face in order to determine the viscous fluxes. Evaluations of those metrics that cannot be computed using the above formulas will be discussed later. 


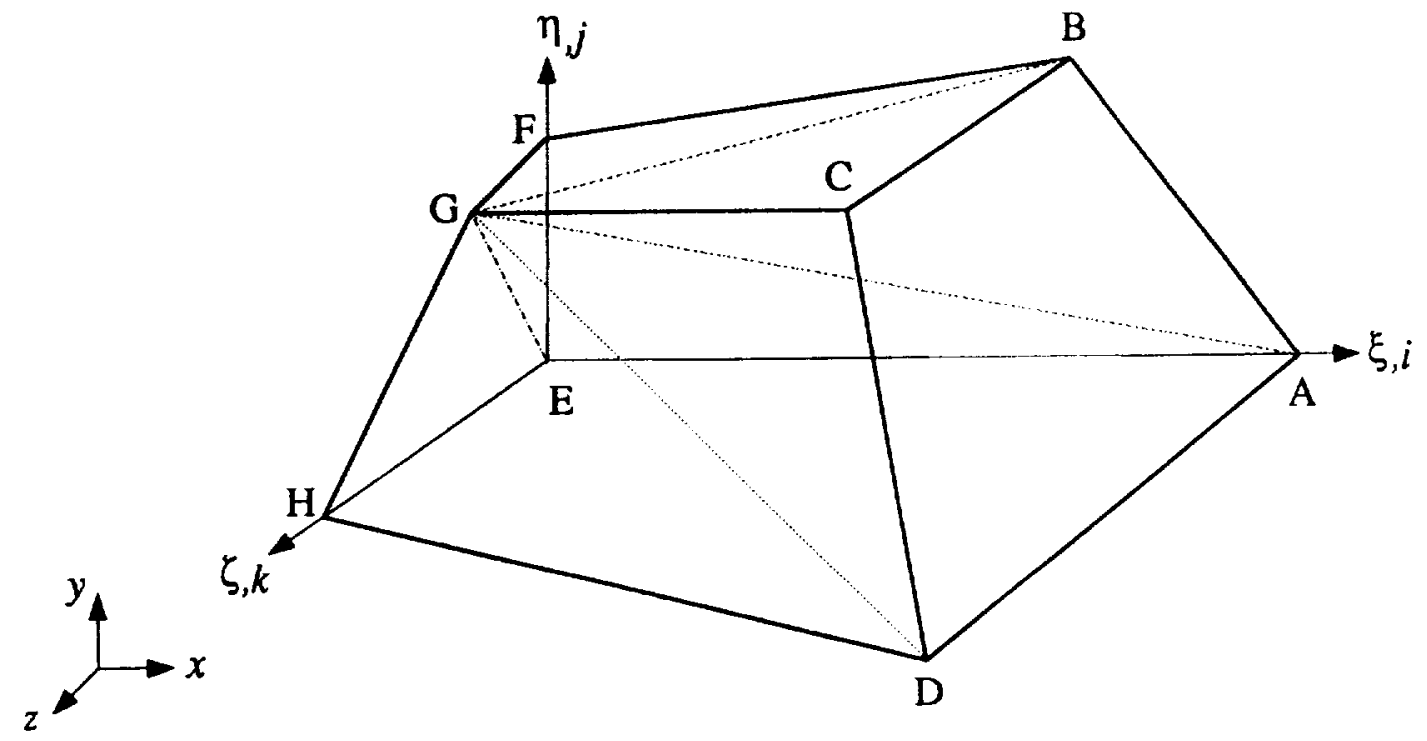

Figure 7-2. Cell Volume Decomposition

The volume of the hexahedron is evaluated as follows ${ }^{[31]}$. The hexahedron can be decomposed into three pyramids as shown in Fig. 7-2. The bases of the three pyramids are ABCD, ABFE and ADHE and they have a common apex at $G$. The volume of each pyramid is one-third the height times the area of the base. Therefore the volume of the hexahedron can be written as

$$
\frac{1}{J}=\frac{1}{3} \overrightarrow{\mathrm{AG}} \cdot\left[\frac{1}{2} \overrightarrow{\mathrm{AC}} \times \overrightarrow{\mathrm{DB}}+\frac{1}{2} \overrightarrow{\mathrm{DE}} \times \overrightarrow{\mathrm{AH}}+\frac{1}{2} \overrightarrow{\mathrm{EB}} \times \overrightarrow{\mathrm{AF}}\right]
$$

The notation $\overrightarrow{A G}$, for example, refers to the vector from $A$ to $G$ pointing towards $G$. The vectors on the pyramid bases are defined such that the directed face areas are pointing into the cell. This ensures that the resulting volume is a positive number. Since a hexahedron has four diagonals, its volume can be evaluated four different ways depending on the choice of the diagonal. In this study the average of these four volumes is used as the volume of the hexahedron.

Now we present the evaluation of metrics that are needed to compute the viscous fluxes but, cannot be computed using the formulas given above. Let us consider the constant $\xi$ face $i+\frac{1}{2}, j, k$. The metric quantities $\eta_{x} / J, \eta_{y} / J, \eta_{z} / J, \zeta_{x} / J, \zeta_{y} / J$ and $\zeta_{z} / J$ at this face are evaluated as an average of the neighboring face values. Thus 


$$
\begin{aligned}
& \left(\frac{\eta_{\theta}}{J}\right)_{i+\frac{1}{2}, j, k}=\frac{1}{4}\left[\left(\frac{\eta_{\theta}}{J}\right)_{i, j+\frac{1}{2}, k}+\left(\frac{\eta_{\theta}}{J}\right)_{i, j+\frac{1}{2}, k}+\left(\frac{\eta_{\theta}}{J}\right)_{i, j-\frac{1}{2}, k}+\left(\frac{\eta_{\theta}}{J}\right)_{i, j-\frac{1}{2}, k}\right] \\
& \left(\frac{\zeta_{\theta}}{J}\right)_{i+\frac{1}{2}, j, k}=\frac{1}{4}\left[\left(\frac{\zeta_{\theta}}{J}\right)_{i, j, k+\frac{1}{2}}+\left(\frac{\zeta_{\theta}}{J}\right)_{i, j, k+\frac{1}{2}}+\left(\frac{\zeta_{\theta}}{J}\right)_{i, j, k-\frac{1}{2}}+\left(\frac{\zeta_{\theta}}{J}\right)_{i, j, k-\frac{1}{2}}\right]
\end{aligned}
$$

where $\theta$ stand for $x, y$ or $z$. It is also necessary to compute a volume associated with the cell face in order to evaluate the viscous terms on the left hand side of the governing equations. This volume is evaluated as an average of the neighboring volumes. Thus

$$
J_{i+\frac{1}{2}, j, k}=\frac{J_{i, j, k}+J_{i+1, j, k}}{2}
$$

where $J$ is the reciprocal of the volume. Similarly the metrics on the other cell-interfaces are evaluated. 


\section{Boundary Conditions}

A major hurdle in simulating vortex breakdown, especially the spiral-type breakdown, is the numerical modelling of boundary conditions consistent with experiments. In the past we were able to simulate bubble-type breakdown by specifying that the flow is essentially inviscid near the outer edge of the computational domain ${ }^{[22]}$. Other investigators have simulated similar bubble-type breakdown by specifying pressure distribution along the outer edge ${ }^{[32]}$. In the present study, we assume that the outer edges of the computational domain are reasonably far away from the breakdown region. Hence the flow in this region is considered to be inviscid, steady and reasonably benign. The boundary conditions that are used are based on the characteristic variables $^{[33]}$. These boundary conditions are developed by assuming that the flow is locally one-dimensional and perpendicular to the boundary, i.e., the derivatives along the surface of the boundary are neglected. With the above assumptions, the following characteristic form of the Euler equations can be derived at a constant $r$ boundary, where $r$ stands for $\xi, \eta$ or $\zeta$.

$$
\begin{array}{lll}
\frac{d s}{d t}=0 & \text { along } & \frac{d r}{d t}=\bar{u} \\
\frac{d \mathcal{V}}{d t}=0 & \text { along } & \frac{d r}{d t}=\bar{u} \\
\frac{d \bar{u}}{d t} \pm \frac{1}{\rho a} \frac{d p}{d t}=0 & \text { along } & \left(\frac{d r}{d t}\right)^{ \pm}=\bar{u} \pm a
\end{array}
$$

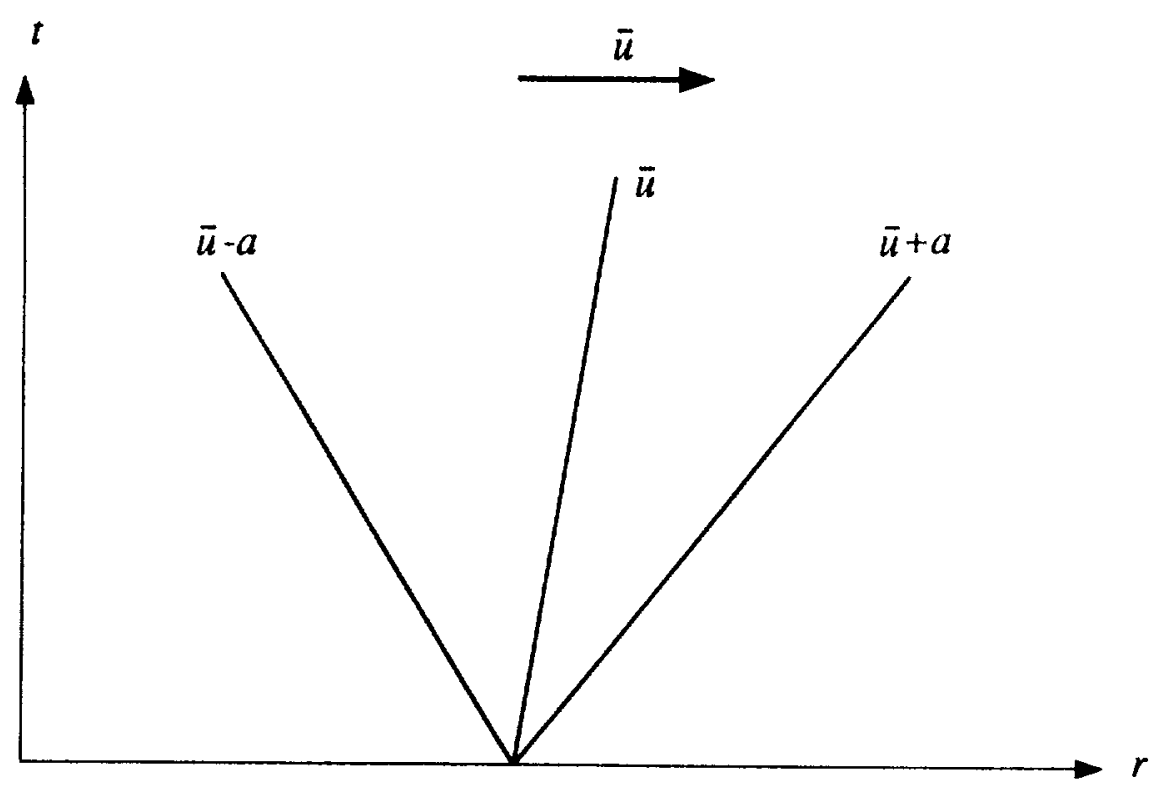

Figure 8-1. Characteristic Waves in a Flow 
where $s$ is the entropy, $\bar{i}$ and $\mathcal{V}$ are the component of velocity normal and tangential to the boundary respectively. The normal velocity is (Eq. 5-36)

$$
\bar{u}=u \bar{r}_{x}+v \bar{r}_{y}+w \bar{r}_{z}
$$

A sketch of the characteristic paths is given Fig. 8-1. According to Eq. 8-1a and Eq. 8-1b, the entropy and tangential velocity, respectively, are convected along the direction of $\bar{u}$. Assuming that the flow is locally homentropic, Eq. 8-1c. can be written as

$$
\frac{d \mathrm{R}^{ \pm}}{d t}=0 \quad \text { along } \quad\left(\frac{d r}{d t}\right)^{ \pm}=\bar{u} \pm a
$$

where

$$
\mathbf{R}^{ \pm} \equiv \bar{u} \pm \frac{2 a}{\sigma}
$$

are the Riemann variables. The boundary conditions are applied by specifying appropriate conditions at a set of phaintom or ghost cell around the boundary. The characteristic equations are used to update the variables at these ghost cells. The ghost cells are updated explicitly at the end of each time step.

For this specific problem the incoming-face is either subsonic or supersonic inflow and the outgoing-face is either subsonic or supersonic outflow. Conditions at a side-faces could be any one or a combination of the above. Therefore the boundary conditions described are specialized for the following four cases.

1. Supersonic inflow

2. Supersonic outflow

3. Subsonic inflow

4. Subsonic outflow

\section{Supersonic Inflow}

For supersonic flows all the eigenvalues have the same sign. Hence, since the flow is coming into the computational domain all the flow variables in the ghost cells are specified.

\section{Supersonic Outflow}

Again, since the flow is supersonic, all the eigenvalues have the same sign. Since the flow is going out of the computitional domain, all the flow variables are extrapolated from inside the computational domain to the ghost cells. 


\section{Subsonic Inflow}

In subsonic flows four of the eigenvalues have the same sign and one has the opposite sign. For the subsonic inflow case four of the eigenvalues, $\bar{u}+a, \bar{u}, \bar{u}$ and $\bar{u}$ are positive and one, $\bar{u}-a$ is negative (Eq. 5-46). Fig. 8-2 shows a schematic representation of the characteristic waves at a constant $r$ boundary at time level $n+1$. At this time level the $\mathbf{R}^{+}$characteristic can be determined from the conditions at the ghost cell at time level $n$ and the $\mathrm{P}^{-}$characteristic can be determined from the conditions at the first interior cell at the same time level. Thus

$$
\begin{aligned}
& \mathbf{R}^{+}=\bar{u}_{G}^{n}+\frac{2 a_{\mathrm{G}}^{n}}{\sigma} \\
& \mathbf{R}^{-}=\bar{u}_{1}^{n}-\frac{2 a_{1}^{n}}{\sigma}
\end{aligned}
$$

where $G$ denotes the ghost cell and I the first interior cell. The two Riemann variables can be added and subtracted to determine a local normal velocity and speed of sound at the time level $n+1$. This new normal velocity and the speed of sound is assigned as the ghost cell value at time level $n+1$. Therefore we can write

$$
\bar{u}_{\mathrm{G}}^{n+1}=\frac{\mathbf{R}^{+}+\mathbf{R}^{-}}{2}=\frac{\bar{u}_{\mathrm{G}}^{n}+\bar{u}_{1}^{n}}{2}+\frac{a_{\mathrm{C}_{\mathrm{K}}}^{n}-a_{1}^{n}}{\sigma}
$$

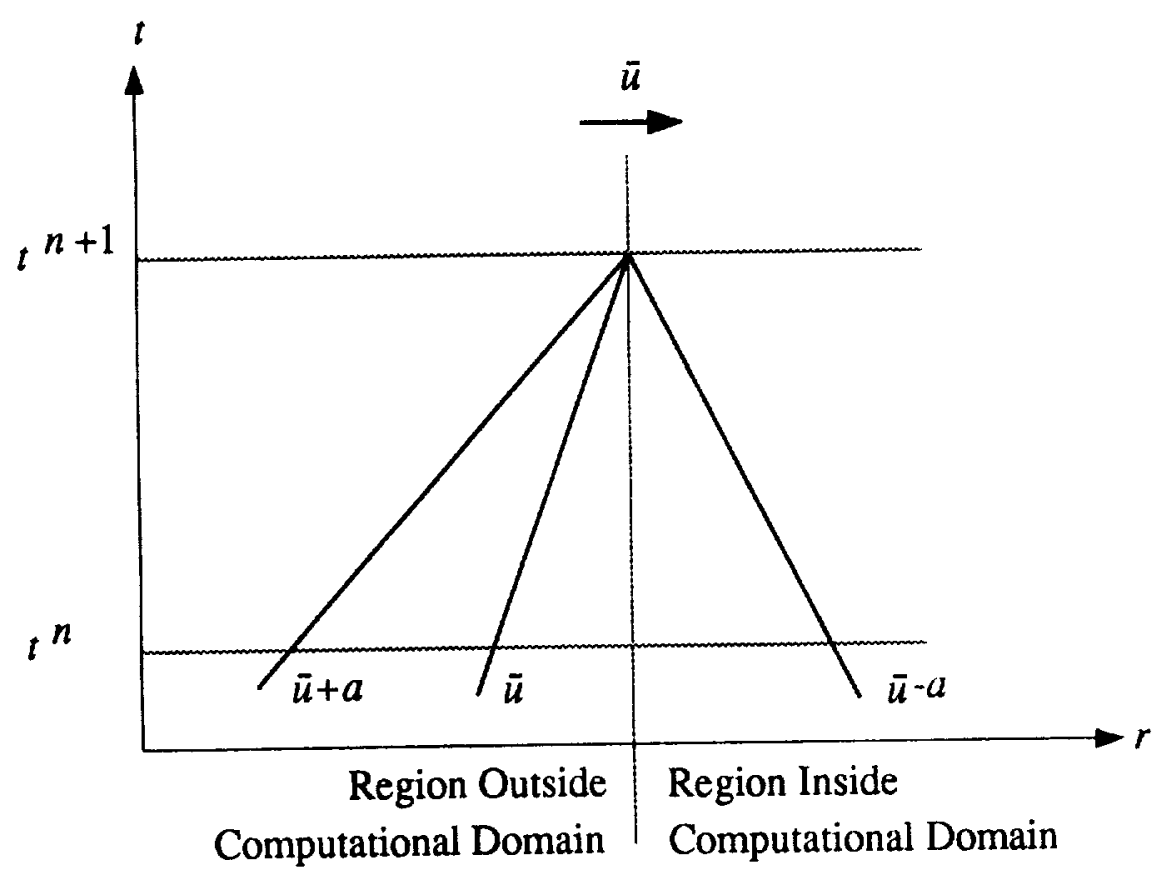

Figure 8-2. Characteristic Waves at an Inflow Boundary 


$$
a_{\mathrm{i}}^{v_{i}^{+}+1}=\frac{\sigma\left(\mathbf{R}^{+}-\mathbf{R}^{-}\right)}{4}=\frac{\sigma\left(\bar{u}_{\mathrm{G}}^{n}-\bar{u}_{1}^{n}\right)}{4}+\frac{a_{\mathrm{i}}^{n}+a_{1}^{n}}{2}
$$

Since the tangential velocity and entropy are convected in the direction of $\bar{u}$, they are held fixed. Therefore

$$
\mathcal{V}_{\mathrm{G}}^{n+1}=\mathcal{V}_{\mathrm{G}}^{n}
$$

and

$$
s_{\mathrm{G}}^{n+1}=s_{\mathrm{G}}^{n}
$$

As shown in Appendix-B.

$$
\begin{aligned}
& \bar{v} \bar{l}_{x}+\bar{w} \bar{m}_{x}=u-\bar{u} \bar{r}_{x} \\
& \bar{v} \bar{l}_{y}+\bar{w} \bar{m}_{y}=v-\bar{u} \bar{r}_{y} \\
& \bar{v} \bar{l}_{z}+\bar{w} \bar{m}_{z}=w-\bar{u} \bar{r}_{z}
\end{aligned}
$$

are the components of the tangential velocity $\mathcal{V}$ in the $x, y$ and $z$ directions. Therefore from Eq. 8-8 we can write,

$$
\begin{aligned}
\left(u-\bar{u} \bar{r}_{x}\right)_{\mathrm{G}}^{n+1} & =\left(u-\bar{u} \bar{r}_{x}\right)_{\mathrm{G}}^{n} \\
\left(v-\bar{u} \bar{r}_{y}\right)_{\mathrm{G}}^{n+1} & =\left(v-\bar{u} \bar{r}_{y}\right)_{\mathrm{G}}^{n} \\
\left(w-\bar{u} \bar{r}_{z}\right)_{\mathrm{G}}^{n+1} & =\left(w-\bar{u} \bar{r}_{z}\right)_{\mathrm{G}}^{n}
\end{aligned}
$$

Eq. $8-11$ can be rearranged as

$$
\begin{array}{r}
u_{G}^{n+1}=u_{G}^{n}+\left(\bar{u}_{G}^{n+1}-\bar{u}_{G}^{n}\right) \bar{r}_{x} \\
v_{G}^{n+1}=v_{G}^{n}+\left(\bar{u}_{G}^{n+1}-\bar{u}_{G}^{n}\right) \bar{r}_{y} \\
w_{G}^{n+1}=w_{G}^{n}+\left(\bar{u}_{G}^{n+1}-\bar{u}_{G}^{n}\right) \bar{r}_{z}
\end{array}
$$

From Eq. 8-9 we can wrile

$$
\left(\frac{p}{\rho^{\gamma}}\right)_{\mathrm{G}}^{n+1}=\left(\frac{p}{\rho^{\gamma}}\right)_{\mathrm{G}}^{n}
$$

From Eqs. 8-7 and 8-13 the total energy per unit mass $e$ and the density $\rho$ can be easily determined. 


\section{Subsonic Outflow}

Subsonic outflow conditions can be derived in a similar manner as subsonic inflow conditions. Fig. 8-3 shows the characteristic waves at a subsonic outflow boundary. At time level $n+1$ the $\mathbf{R}^{+}$characteristic is determined from the conditions at the interior cell adjacent to the boundary cell at time level $n$ and the $\mathbf{R}^{-}$characteristic is determined from the conditions at the ghost cell at the same time level. Thus we can write

$$
\begin{aligned}
& \mathbf{R}^{+}=\bar{u}_{1}^{n}+\frac{2 a_{1}^{n}}{\sigma} \\
& \mathbf{R}^{-}=\bar{u}_{\mathrm{G}}^{n}-\frac{2 a_{\mathrm{G}}^{n}}{\sigma}
\end{aligned}
$$

and therefore

$$
\begin{gathered}
\bar{u}_{\mathrm{i}}^{n+1}=\frac{\mathbf{R}^{-}+\mathbf{R}^{+}}{2}=\frac{\bar{u}_{\mathrm{G}}^{n}+\bar{u}_{1}^{n}}{2}-\frac{a_{\mathrm{C}}^{n}-a_{1}^{n}}{\sigma} \\
a_{\mathrm{G}}^{n+1}=\frac{\sigma\left(\mathbf{R}^{-}-\mathbf{R}^{+}\right)}{4}=-\frac{\sigma\left(\bar{u}_{\mathrm{G}}^{n}-\bar{u}_{\mathrm{I}}^{n}\right)}{4}+\frac{a_{\mathrm{C}}^{n}+a_{1}^{n}}{2}
\end{gathered}
$$

The tangential velocity and entropy are extrapolated to the ghost cell, using zeroth order extrapolation, from the interior cell. Therefore

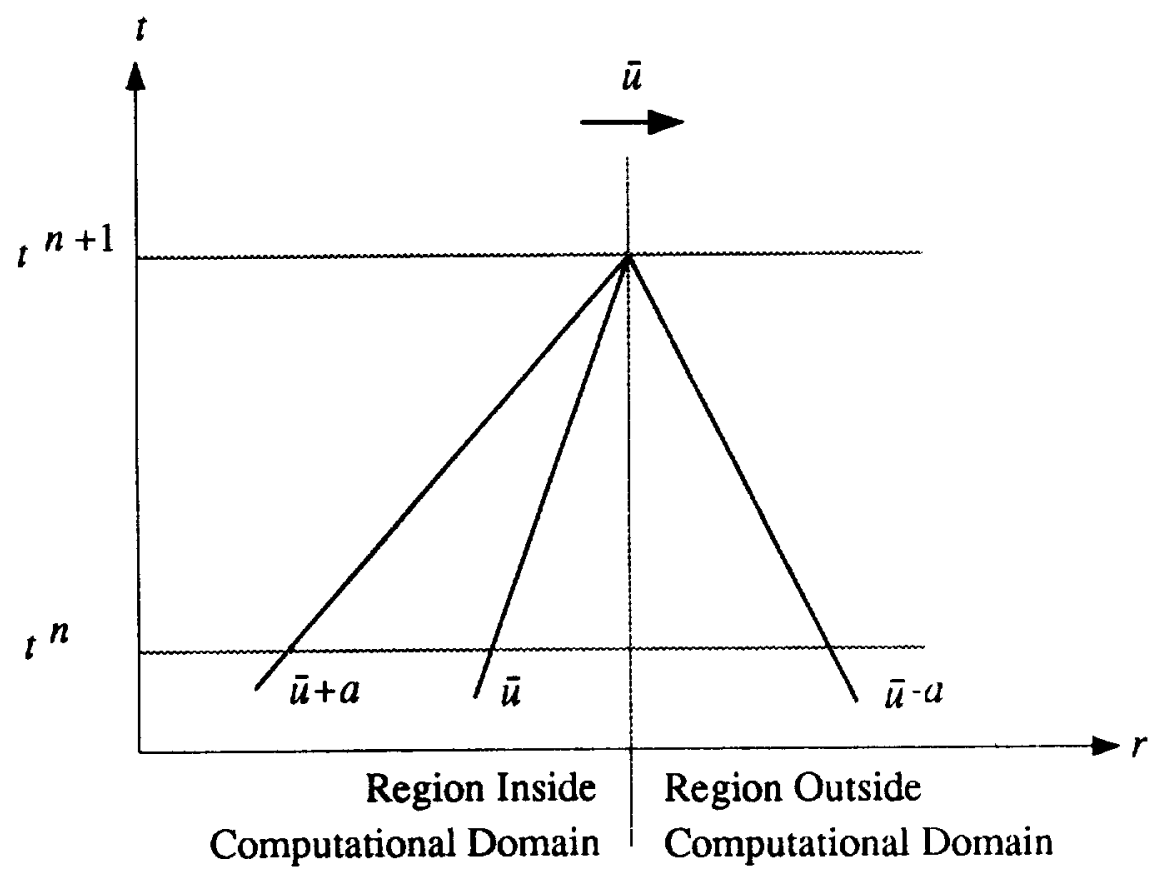

Figure 8-3. Characteristic Waves at an Outflow Boundary 


$$
\mathcal{V}_{\mathrm{i}}^{n+1}=\mathcal{V}_{\mathrm{l}}^{n}
$$

and

$$
s_{\mathrm{G}}^{n+1}=s_{1}^{n}
$$

Similar to the subsonic inflow case it can be shown that

$$
\begin{array}{r}
u_{\mathrm{G}}^{n+1}=u_{1}^{n}+\left(\bar{u}_{\mathrm{G}}^{n+1}-\bar{u}_{1}^{n}\right) \bar{r}_{x} \\
v_{\mathrm{G}}^{n+1}=v_{\mathrm{I}}^{n}+\left(\bar{u}_{\mathrm{G}}^{n+1}-\bar{u}_{\mathrm{I}}^{n}\right) \bar{r}_{y} \\
w_{\mathrm{G}}^{n+1}=w_{1}^{n}+\left(\bar{u}_{\mathrm{G}}^{n+1}-\bar{u}_{1}^{n}\right) \bar{r}_{z}
\end{array}
$$

and

$$
\left(\frac{p}{\rho^{\gamma}}\right)_{\mathrm{G}}^{n+1}=\left(\frac{p}{\rho^{\gamma}}\right)_{\mathrm{I}}^{n}
$$

Now we have all the concitions needed to write the complete set of algebraic equations. 


\section{Solution Procedure}

In this section we describe the procedure for solving the algebraic equations obtained by discretizing the governing equations. By substituting for the flux Jacobians from Eqs. 5-33, 5-34, 6-18 and 6-21 in Eq. 4-13 we get, for a cell $(i, j, k)$,

$$
\begin{array}{r}
\begin{array}{c}
\mathrm{I} \Delta Q_{i, j, k} \\
+J \Delta t\left\{\left(\mathrm{~T}_{\xi} \Pi_{\xi}^{+} \mathrm{T}_{\xi}^{-1}\right)_{i+\frac{1}{2}, j, k} \Delta Q_{i, j, k}\right.
\end{array}+\left(\mathrm{T}_{\xi} \Pi_{\xi}^{-} \mathrm{T}_{\xi}^{-1}\right)_{i+\frac{1}{2}, j, k} \Delta Q_{i+1, j, k} \\
-\left(\mathrm{T}_{\xi} \Pi_{\xi}^{+} \mathrm{T}_{\xi}^{-1}\right)_{i-\frac{1}{2}, j, k} \Delta Q_{i-1, j, k}-\left(\mathrm{T}_{\xi} \Pi_{\xi}^{-} \mathrm{T}_{\xi}^{-1}\right)_{i-\frac{1}{2}, j, k} \Delta Q_{i, j, k} \\
-\left(\mathrm{V}_{\xi}^{+}\right)_{i+\frac{1}{2}, j, k} \Delta Q_{i+1, j, k} \quad+\left(\mathrm{V}_{\xi}^{-}\right)_{i+\frac{1}{2}, j, k} \Delta Q_{i, j, k} \\
\left.+\left(\mathrm{V}_{\xi}^{+}\right)_{i-\frac{1}{2}, j, k} \Delta Q_{i, j, k} \quad-\left(\mathrm{V}_{\xi}^{-}\right)_{i-\frac{1}{2}, j, k} \Delta Q_{i-1, j, k}\right\} \\
=-J \Delta t\left\{E_{i+\frac{1}{2}, j, k}-E_{i-\frac{1}{2}, j, k}+F_{i, j+\frac{1}{2}, k}-F_{i, j-\frac{1}{2}, k}+G_{i, j, k+\frac{1}{2}}-G_{i, j, k-\frac{1}{2}}\right. \\
\left.-\quad-\mathcal{E}_{i+\frac{1}{2}, j, k}+\mathcal{E}_{i-\frac{1}{2}, j, k}-\mathcal{F}_{i, j+\frac{1}{2}, k}+\mathcal{F}_{i, j-\frac{1}{2}, k}-\mathcal{G}_{i, j, k+\frac{1}{2}}+\mathcal{G}_{i, j, k-\frac{1}{2}}\right\}
\end{array}
$$

Eq. 9-1 can be written as

$$
\mathbf{A}_{i} \Delta Q_{i-1, j, k}+\mathbf{B}_{i} \Delta Q_{i, j, k}+\mathbf{C}_{i} \Delta Q_{i+1, j, k}=\mathbf{D}_{i}
$$

where

$$
\begin{aligned}
& \mathbf{A}_{\mathbf{i}}=-J \Delta t\left\{\left(\mathrm{~T}_{\xi} \Pi_{\xi}^{+} \mathrm{T}_{\xi}^{-1}\right)_{i-\frac{1}{2}, j, k}+\left(\mathbf{V}_{\xi}^{-}\right)_{i-\frac{1}{2}, j, k}\right\} \\
& \mathbf{B}_{\mathbf{i}}=\mathbf{I}+J \Delta t\left\{\left(\mathrm{~T}_{\xi} \Pi_{\xi}^{+} \mathbf{T}_{\xi}^{-1}\right)_{i+\frac{1}{2}, j, k}-\left(\mathrm{T}_{\xi} \Pi_{\xi}^{-} \mathbf{T}_{\xi}^{-1}\right)_{i-\frac{1}{2}, j, k}\right. \\
& \left.+\left(\mathbf{v}_{\xi}^{-}\right)_{i+\frac{1}{2}, j, k}+\left(\mathbf{v}_{\xi}^{+}\right)_{i-\frac{1}{2}, j, k}\right\} \\
& \mathbf{C}_{\boldsymbol{i}}=J \Delta t\left\{\left(\mathbf{T}_{\xi} \Pi_{\xi}^{-} \mathbf{T}_{\xi}^{-1}\right)_{i+\frac{1}{2}, j, k}-\left(\mathbf{V}_{\xi}^{+}\right)_{i+\frac{1}{2}, j, k}\right\}
\end{aligned}
$$

are $5 \times 5$ matrices and

$$
\begin{array}{r}
\mathbf{D}_{i}=-J \Delta t\left\{E_{i+\frac{1}{2}, j, k}-E_{i-\frac{1}{2}, j, k}+F_{i, j+\frac{1}{2}, k}-F_{i, j-\frac{1}{2}, k}+G_{i, j, k+\frac{1}{2}}-G_{i, j, k-\frac{1}{2}}\right. \\
\left.-\mathcal{E}_{i+\frac{1}{2}, j, k}+\mathcal{E}_{i-\frac{1}{2}, j, k}-\mathcal{F}_{i, j+\frac{1}{2}, k}+\mathcal{F}_{i, j-\frac{1}{2}, k}-\mathcal{G}_{i, j, k+\frac{1}{2}}+\mathcal{G}_{i, j, k-\frac{1}{2}}\right\}
\end{array}
$$


is a vector with 5 elements. By ordering these equations in the increasing $i$ direction, we get a block tri-diagonal system of equations that can be written as

$$
\begin{aligned}
\mathbf{B}_{1} \Delta Q_{1, j, k}+\mathbf{C}_{1} \Delta Q_{2, j, k} & =\mathbf{D}_{1} & \text { for } i=1 \\
\mathbf{A}_{i} \Delta Q_{i-1, j, k}+\mathbf{B}_{i} \Delta Q_{i, j, k}+\mathbf{C}_{i} \Delta Q_{i+1, j, k} & =\mathbf{D}_{i} & \text { for } i=2, I-1 \\
\mathbf{A}_{I} \Delta Q_{I-1, j, k}+\mathbf{B}_{l} \Delta Q_{I, j, k} & =\mathbf{D}_{l} & \text { for } i=I
\end{aligned}
$$

where $i=1$ is the first interior cell and $i=I$ is the last interior cell in the $i$-direction at any $j, k$ location. Similar sys em of equations can be constructed for the other two directions, $j$ and $k$ Eqs. 3-10b and 3-10c, of the alternating direction sequence where, the resulting equations are arranged in their respective direction.

\section{Thomas Algorithm}

Each of the block tri-diagonal system is solved using the Thomas algorithm where the solution is obtained in two stages. In the first stage a set of auxiliary quantities $\mathbf{E}$ and $\mathbf{F}$ are computed using a set of forward recursive formula starting at $i=1$. In the second stage the required solution is obtained from a backward recursive relation starting at $i=I$. Skipping the derivation, these formula are, for the forward sweep,

$$
\begin{aligned}
& \mathbf{E}_{1}=-\mathbf{B}_{1}^{-1} \mathbf{C}_{1} \\
& \mathbf{F}_{1}=: \mathbf{B}_{1}^{-1} \mathbf{D}_{1} \\
& \mathbf{E}_{\mathbf{i}}:=-\left(\mathrm{A}_{\mathbf{i}} \mathbf{E}_{\boldsymbol{i}-1}+\mathbf{B}_{\boldsymbol{i}}\right)^{-1} \mathbf{C}_{\boldsymbol{i}} \\
& \left.\mathbf{F}_{\boldsymbol{i}}==\left(\mathbf{A}_{\boldsymbol{i}} \mathbf{E}_{\boldsymbol{i}-1}+\mathbf{B}_{\boldsymbol{i}}\right)^{-1}\left(\mathbf{D}_{\boldsymbol{i}}-\mathbf{A}_{\boldsymbol{i}} \mathbf{F}_{\boldsymbol{i}-1}\right)\right\} \text { for } i=2, I
\end{aligned}
$$

and for the backward sweep

$$
\begin{aligned}
& \Delta Q_{I, j, k}=\mathbf{F}_{I} \\
& \Delta Q_{i, j, k}=\mathbf{E}_{i} \Delta Q_{i+1, j, k}+\mathbf{F}_{i} \quad \text { for } i=I-1,1
\end{aligned}
$$

The inversion of the $5 \times 5$ blocks in Eq. 9-6 is done by Gaussian elimination. This procedure is applied at all the $j, k$ locations to obtain the $\Delta Q_{i, j, k}$ for the whole flowfield.

The solution vector $\angle Q_{i, j, k}$ arranged in the $j$ direction at each $k, i$ becomes the right hand side of the second sequence and the resulting equations are solved in a similar manner as described above. This procedure is repeated for the third sequence. From the final $\Delta Q$ the solution at the next step is obtained form Eq. 3-11 which is

$$
Q_{i, j, k}^{n+1}=Q_{i, j, k}^{n}+\Delta Q_{i, j, k}
$$


For steady state problems, this process is repeated until the desired convergence criteria is satisfied. For unsteady problems this process is stopped at the desired time level.

\section{Time Step}

The time step used to advance the solution is determined as follows. The time step limit at a cell is given by the following equation

$$
\frac{1}{\Delta t_{i, j, k}}=\frac{1}{C F L}\left(\frac{1}{\Delta t_{\xi}}+\frac{1}{\Delta t_{\eta}}+\frac{1}{\Delta t_{\zeta}}\right)
$$

where

$$
\begin{aligned}
& \frac{1}{\Delta t_{\xi}}=J_{i, j, k} \frac{1}{2}\left[(|\bar{u}|+a)_{i+\frac{1}{2}, j, k}\left(\frac{|\nabla \xi|}{J}\right)_{i+\frac{1}{2}, j, k}+(|\bar{u}|+a)_{i-\frac{1}{2}, j, k}\left(\frac{|\nabla \xi|}{J}\right)_{i-\frac{1}{2}, j, k}\right] \\
& \frac{1}{\Delta t_{\eta}}=J_{i, j, k} \frac{1}{2}\left[(|\bar{u}|+a)_{i, j+\frac{1}{2}, k}\left(\frac{|\nabla \eta|}{J}\right)_{i, j+\frac{1}{2}, k}+(|\bar{u}|+a)_{i, j-\frac{1}{2}, k}\left(\frac{|\nabla \eta|}{J}\right)_{i, j-\frac{1}{2}, k}\right] \\
& \frac{1}{\Delta t_{\zeta}}=J_{i, j, k} \frac{1}{2}\left[(|\bar{u}|+a)_{i, j, k+\frac{1}{2}}\left(\frac{|\nabla \zeta|}{J}\right)_{i, j, k+\frac{1}{2}}+(|\bar{u}|+a)_{i, j, k-\frac{1}{2}}\left(\frac{|\nabla \zeta|}{J}\right)_{i, j, k-\frac{1}{2}}\right]
\end{aligned}
$$

and the $C F L$ used in this study is

$$
1<C F L \leq 10
$$

The flow variables at cell-interfaces are determined by averaging the cell-average quantities from the adjacent cells. For steady state problems the solution at each cell is advanced using the local time step. For unsteady problems a global minimum time step is used.

\section{Multigrid Acceleration}

For steady state problems, the rate of convergence of the steady-state residual $R-\mathcal{R}$ can be improved using a full approximation multigrid acceleration technique ${ }^{[34][35]}$. Although this acceleration technique was originally developed for elliptic problems, it has been successively used for a variety of problems including transonic flow problems that contains shocks and other discontinuities. In this technique, starting with the finest grid, the problem is solved on a series of successively coarser grids and the correction obtained on each of the coarser grids is passed back successively and eventually to the finest grid. The coarse grids reduce the low frequencies (with respect to the fine grid) errors quicker than the fine grid. Although, for multigrid method, computational cost per iteration is higher than that of single grid methods, the gain in the rate of convergence far offsets the additional computational overhead. The implementation of the multigrid acceleration technique is presented below. 
Let

$$
\mathcal{L}_{h}\left(Q_{h}\right)=f_{h}
$$

represent the problem that we intend to solve where $\mathcal{L}_{h}$ is the nonlinear discrete operator that we obtain by discretizing the Navier-Stokes equations, as described in the previous sections and $f_{h}$ is the forcing function associated with the equations. For the Navier-Stokes equations, considered here, $f_{h}=0$. However we will retain it for generality. The subscript $h$ denotes the finest grid and $2 h, 4 h$ etc. denotes successively coarser grids where each coarse grid is obtained by removing every other grid point from the preceding fine grid. We seek a solution $Q_{h}$ such that the residual $f_{h}-\mathcal{L}_{h}\left(Q_{h}\right)$ goes to zero after a number of iterations of the solution scheme (ADI scheme in this particular work). After $N_{h}$ iterations of the solution scheme, on grid $h$, let $Q_{h}^{\prime}$ be the solution. The error in the solution can be written as

$$
\nu_{h}=Q_{h}-Q_{h}^{\prime}
$$

or we can write

$$
Q_{h}=Q_{h}^{\prime}+\nu_{h}
$$

where $\nu_{h}$ is the correction that has to be added to the fine grid solution to obtain the exact solution. In order to obtain this corection, we solve an equivalent problem on the next coarse grid $2 h$. The equivalent problem on grid $2 h$ can be written as

$$
\mathcal{L}_{2 h}\left(Q_{2 h}^{\prime}\right)=f_{2 h}
$$

where,

$$
f_{2 h}=\underset{2 h}{\downarrow}\left[f_{h}-\mathcal{L}_{h}\left(Q_{h}^{\prime}\right)\right]+\mathcal{L}_{2 h}\left(\begin{array}{l}
h \\
\underset{2 h}{h} Q_{h}^{\prime}
\end{array}\right)
$$

$h$

$\downarrow$ represents an operator hat restricts quantities from grid $h$ on to the next courser grid $2 h$. The exact form of the restriction operator will be presented later. The forcing function $f_{2 h}$ ensures that the coarse grid problem, Eq. 9-15, is driven by the residual of the finest grid. Starting with ${ }_{2 h}^{h} Q_{h}^{\prime}$ as an initial guess, the solution scheme is applied to this new problem and let $Q_{2 h}^{\prime \prime}$ be the solution after $N_{2 h}$ iterations. Again we can write

$$
Q_{2 h}^{\prime}=Q_{2 h}^{\prime \prime}+\nu_{2 h}
$$


and we seek to obtain the correction $\nu_{2 h}$ by solving an equivalent problem on the next coarse grid 4h. We construct the equivalent problem

$$
\mathcal{L}_{4 h}\left(Q_{4 h}^{\prime \prime}\right)=f_{4 h}
$$

on the next coarse grid $4 h$ where,

$$
f_{4 h}=\downarrow_{4 h}^{2 h}\left[f_{2 h}-\mathcal{L}_{2 h}\left(Q_{2 h}^{\prime \prime}\right)\right]+\mathcal{L}_{2 h}\left(\underset{4 h}{\stackrel{2 h}{\downarrow} Q_{2 h}^{\prime \prime}}\right)
$$

Several iterations of the solution can be performed to Eq. 9-18. Let $Q_{4 h}^{\prime \prime \prime}$ be the solution after $N_{4 h}$ iterations. This process can be continued on as many successively coarser grids as desired or possible. At the end of this process, we proceed to pass (prolongate) the corrections successively from the coarse grid to the next finer grid, using a suitable interpolation function, all the way to the finest grid. This is done in the following manner. Let us assume that the coarsest level that we down to is $4 h$ and that we were able to solve the problem exactly, i.e., we found $Q_{4 h}^{\prime \prime}$. Now we prolongate the correction $Q_{4 h}^{\prime \prime}-{\underset{4 h}{2 h}}_{2 h}^{\prime \prime}$ from this grid to the next finer grid $2 h$ and the corrected solution $\bar{Q}_{2 h}^{\prime \prime}$ on grid $2 h$ can be written as

$$
\bar{Q}_{2 h}^{\prime \prime}=Q_{2 h}^{\prime \prime}+\bar{\nu}_{2 h}
$$

where

$$
\bar{\nu}_{2 h}=\uparrow_{4 h}^{2 h}\left[Q_{4 h}^{\prime \prime}-\underset{4 h}{2 h} Q_{2 h}^{\prime \prime}\right]
$$

where $\uparrow_{4 h}^{2 h}$ is a prolongation operator. The functional form of this operator will be presented later. Starting with the corrected solution Eq. 9-15 can be iterated several times to dampen the errors introduced by the prolongation operator and obtain a new solution on grid $2 h$. Let $\bar{Q}_{2 h}^{\prime}$ be the solution after $\mathcal{N}_{2 h}$ iterations of the solution scheme. The correction $\bar{Q}_{2 h}^{\prime}-{ }_{2 h}^{h} Q_{h}^{\prime}$ is prolongated to the finest grid and the corrected solution on gird $h$ can be written as

$$
\bar{Q}_{h}=Q_{h}^{\prime}+\bar{\nu}_{h}
$$

where

$$
\bar{\nu}_{h}=\uparrow_{2 h}^{h}\left[\bar{Q}_{2 h}^{\prime}-\underset{2 h}{\downarrow} Q_{h}^{\prime}\right]
$$

This process is repeated until the solution converges. 


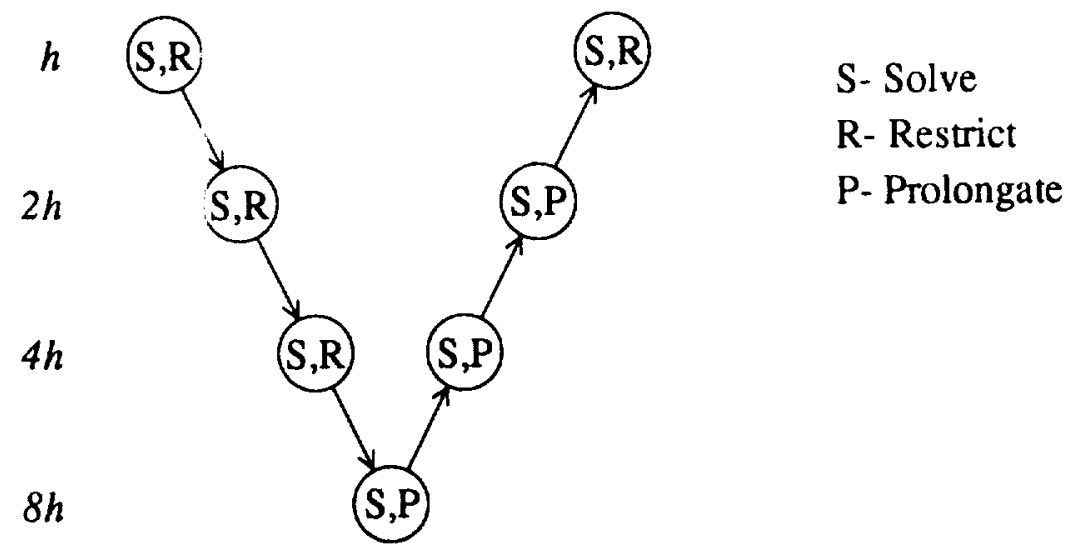

Figure 9-1. Multigrid V-Cycle

The sequence in which the transfers are done between grids can be done in a variety of ways. The usual strategy is to use the V-cycle or the W-cycle. In the V-cycle, the problem is solved on successively coarser grids, but driven by the finest grid residual, followed by the transfer of the correction and solution on successively finer grids all the way to the finest grid. Fig. 9-1a shows a 4-level V-cycle. In the W-iycle, intermediate V-cycles are performed on the coarser grids. Fig. 9$2 \mathrm{~b}$ shows a 4-level $\mathrm{W}$-cycle. Computationally $\mathrm{W}$-cycles are about $50 \%$ more expensive than $\mathrm{V}$-cycle, however, $\mathrm{W}$-cycles are considered more robust. The number of iterations of the solver required on each grid, $N_{h}, N_{2 h} \ldots, \mathcal{N}_{2 h} \ldots$ etc., to get the optimum performance, depends on the problem. In general the choice is to do more iterations on the coarser grids than on the finer ones.

\section{Restriction Operator}

The summation of the residuals of the fine grid cells, that make up a coarse grid cell is used as the restricted residual at that cell. Therefore, since there are 8 fine cells per course cell (Refer to Eq. 9-16),

$$
\underset{\downarrow}{\downarrow}\left[f_{h}-\mathcal{L}_{h}\left(Q_{h}^{\prime}\right)\right]=\sum_{\text {cell }=1}^{8}\left[f_{h}-\mathcal{L}_{h}\left(Q_{h}^{\prime}\right)\right]
$$

The conserved variables are restricted by a volume weighted average. Thus 


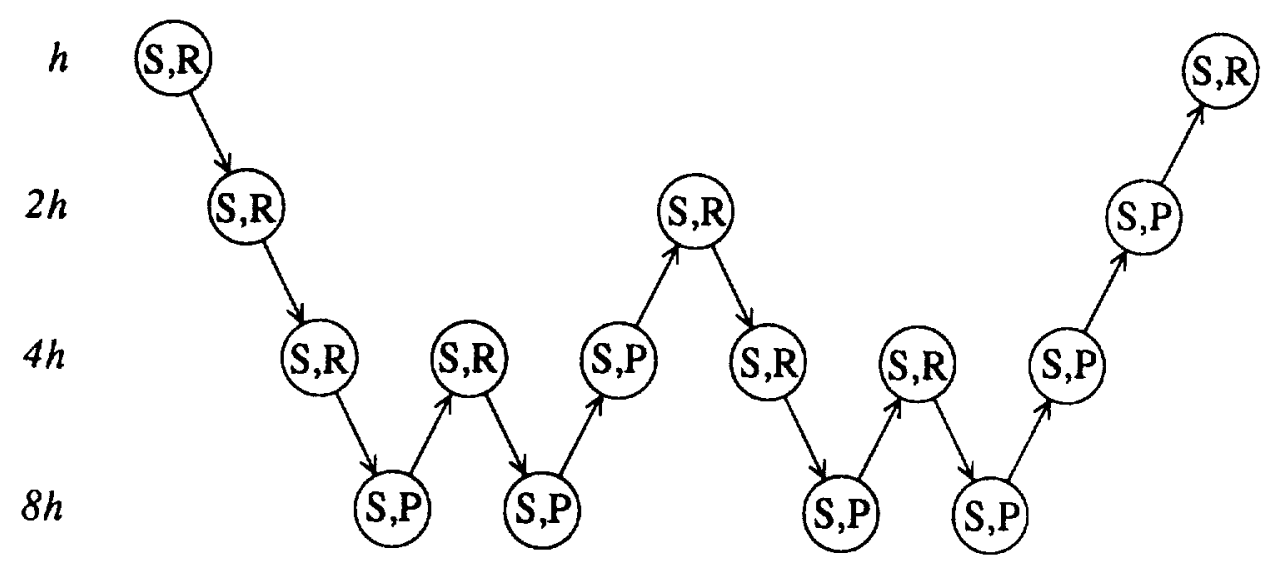

Figure 9-2. Multigrid W-Cycle

$$
{ }_{2 h} Q_{h}^{\prime}=\frac{\sum_{\text {cell }=1}^{8} Q_{h}^{\prime} / J_{h}}{\sum_{\text {cell }=1}^{8} 1 / J_{h}}
$$

\section{Prolongation Operator}

The corrections are prolongated to the fine grid cells using a tri-linear interpolation from the neighboring coarse grid cells. The interpolation function used to prolongate coarse grid cell-center values at $A, B, C, D, E, F, G$, and $H$ to a fine grid cell-center ' $a$ ', as shown in Fig. 9-3, can be written as

$$
{ }_{2 h}^{h} \delta Q_{2 h}=\frac{27}{64} \delta Q_{\mathrm{A}}+\frac{9}{64}\left(\delta Q_{\mathrm{B}}+\delta Q_{\mathrm{D}}+\delta Q_{\mathrm{E}}\right)+\frac{3}{64}\left(\delta Q_{\mathrm{C}}+\delta Q_{\mathrm{F}}+\delta Q_{\mathrm{H}}\right)+\frac{1}{64} \delta Q_{\mathrm{G}}
$$

where (Refer to Eq. 9-22),

$$
\delta Q_{2 h}=\bar{Q}_{2 h}^{\prime}-\stackrel{\downarrow}{2 h}_{h}^{h} Q_{h}^{\prime}
$$




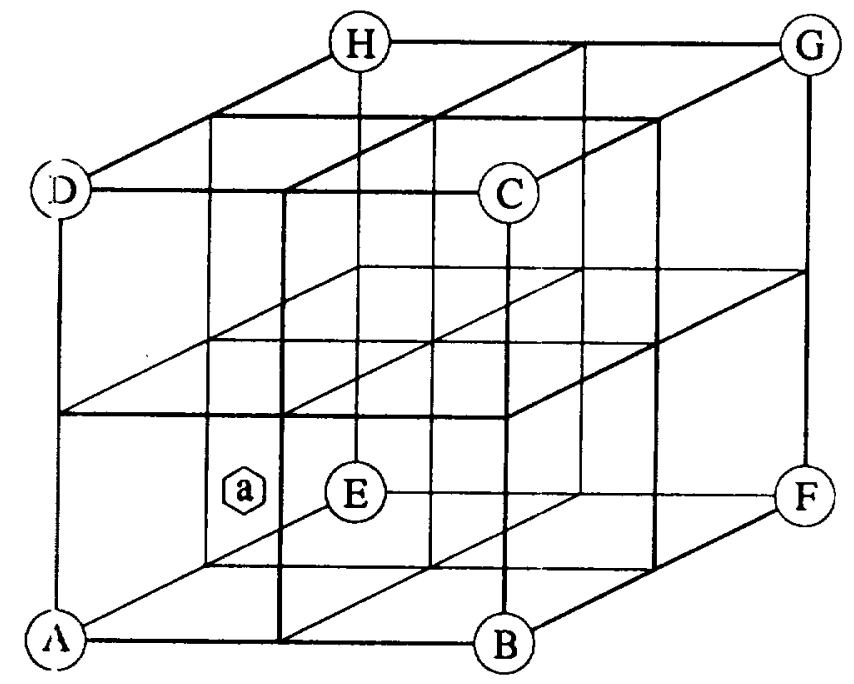

\section{$\bigcirc$ Ciarse Grid Cell-center $\bigcirc$ Fine Grid Cell-center}

Figure 9-3. Coarse to Fine grid transfer

\section{Smoothing of Residuals and Corrections before transfer} below

After restricting to a coarse grid, the residuals are smoothed by the implicit operator given

$$
\left(1-\varepsilon_{\xi} \delta_{\xi \xi}\right)\left(1-\varepsilon_{\eta} \delta_{\eta \eta}\right)\left(1-\varepsilon_{\zeta} \delta_{\zeta \zeta}\right) \ddot{U}=U
$$

where $\delta_{\xi \xi}, \delta_{\eta \eta}$ and $\delta_{\zeta \zeta}$ are central- second-difference operators in the $i, j$ and $k$ directions respectively. Thus, for example,

$$
\delta_{\xi \xi} \bar{U}=\bar{U}_{i-1, j, k}-2 \bar{U}_{i, j, k}+\bar{U}_{i+1, j, k}
$$

$U$ is the quantity that needs to be smoothed and $\bar{U}$ is the result of the smoothing. For example after restricting from grid $l$ to $2 h$ the residual

$$
U=\stackrel{h}{\downarrow}\left[f_{h}-\mathcal{L}_{h}\left(Q_{h}^{\prime}\right)\right]
$$

(Refer to Eq. 9-16) is smoothed. Similarly before prolongation from grid $2 h$ to $h$ the corrections

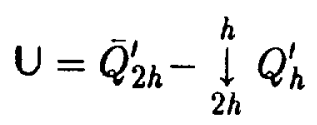


(Refer to Eq. 9-22) are smoothed ${ }^{[36]} . \varepsilon_{\xi}, \varepsilon_{\eta}$ and $\varepsilon_{\zeta}$ are free parameters that are used to control the amount of smoothing. Typically

$$
0<\varepsilon_{\xi}, \varepsilon_{\eta}, \varepsilon_{\zeta}<1
$$

\section{Full Multigrid}

In order to obtain a good initial approximation for the finest grid, the problem, Eq. 9-12, is first iterated on a coarsest grid and the solution in prolongated to the next finer grid, several multigrid cycles are performed between these two levels and the solution is prolongated to the next finer grid. This process is repeated until the finest grid is reached where the solution is used as the initial guess and the multigrid process is continued. These preliminary multigrid cycles are inexpensive and results in a lower initial residual for the finest grid. This helps to drive the final residual to machine-zero in a fewer number of interations. 


\section{Flow Conditions}

\section{Fluid Constants}

The fluid in this study is assumed to be air and the following constants are used.

Prandtl Number, $\operatorname{Pr}=1.0$

Ratio of specific herts, $\gamma=1.4$

Exponent of Temperitture to evaluate viscosity, $\omega=1.0$

\section{Incoming Flow Profiles}

The reference length used in the non-dimensionalization of the governing equations is the radius of the vortex-core at the incoming-face, $x=0$. The Reynolds number is based on this reference length and a reference velocity $=1$. The initial velocity profiles are the same one that has been used by several previous investigators including the present authors ${ }^{[2]}$ in their axisymmetric studies. They can be written in the cartesian coordinate system as follows

$$
\begin{aligned}
u & =M_{\infty} \\
v & =-M_{\infty} \mathcal{V}_{z} / d \\
w & =M_{\infty} \mathcal{V} y / d
\end{aligned}
$$

where $\mathcal{V}$ is the velocity component in the $y z$-plane, which is given as

$$
\begin{array}{lr}
\mathcal{V}=\mathcal{S} d\left(2-d^{2}\right), & 0 \leq d \leq 1 \\
\mathcal{V}=\mathcal{S} / d, & d \geq 1
\end{array}
$$

where

$$
d=\left(y^{2}+z^{2}\right)^{1 / 2}
$$

is the radial distance from the center of the vortex and $\mathcal{S}$, the swirl parameter, is the circumferential velocity at the edge of the vortex-core. $\mathcal{S}$ is a measure of the strength of the incoming vortex. Like $M_{\infty}$ and $R e$, it is a free parameter of the problem.

In order to determini: the profiles of the thermodynamic variables $\rho$ and $e$ a suitable total enthalpy was calculated based on the maximum total velocity and a temperature $=1$. It can be shown that the maximum velocity occurs at $d=(2 / 3)^{1 / 2}$ and the maximum velocity can be written as

$$
V_{\max }^{2}=M_{\infty}^{2}\left(1+\mathcal{S}^{2} 4(2 / 3)^{3}\right)
$$

Therefore the total enthalpy can be written as

$$
H=\gamma / \sigma+V_{\max }^{2} / 2
$$


From the total enthalpy and the velocity, the temperature can be written as

$$
T=\frac{\sigma}{\gamma}\left(H-\frac{u^{2}+v^{2}+w^{2}}{2}\right)
$$

and the energy is

$$
e=\frac{T}{\sigma}+\frac{u^{2}+v^{2}+w^{2}}{2}
$$

Assuming that there is no change in entropy, the density can be written as

$$
\rho=T^{1 / \sigma}
$$

For $M_{\infty}=0.1$ and $\mathcal{S}=1$, the profiles of some of the quantities, expressed above, are shown in the Figs 10-1 to 10-6. The quantities shown are plotted along $y$ at $(x, z: 0,0)$. The incoming-flow conditions are used as initial conditions at all the $x$ locations.

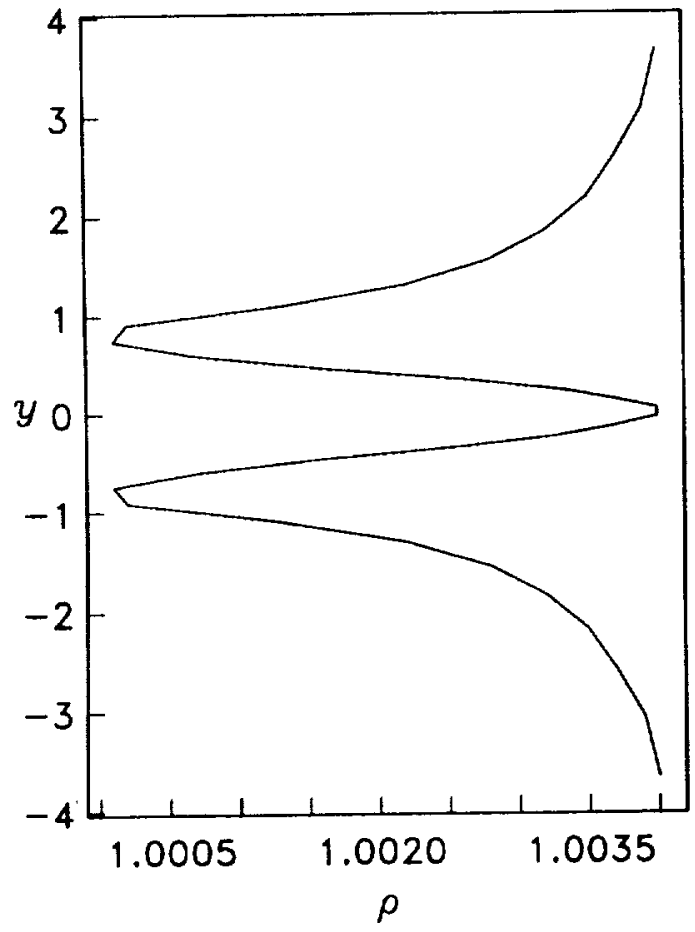

Figure 10-1. Density Profile

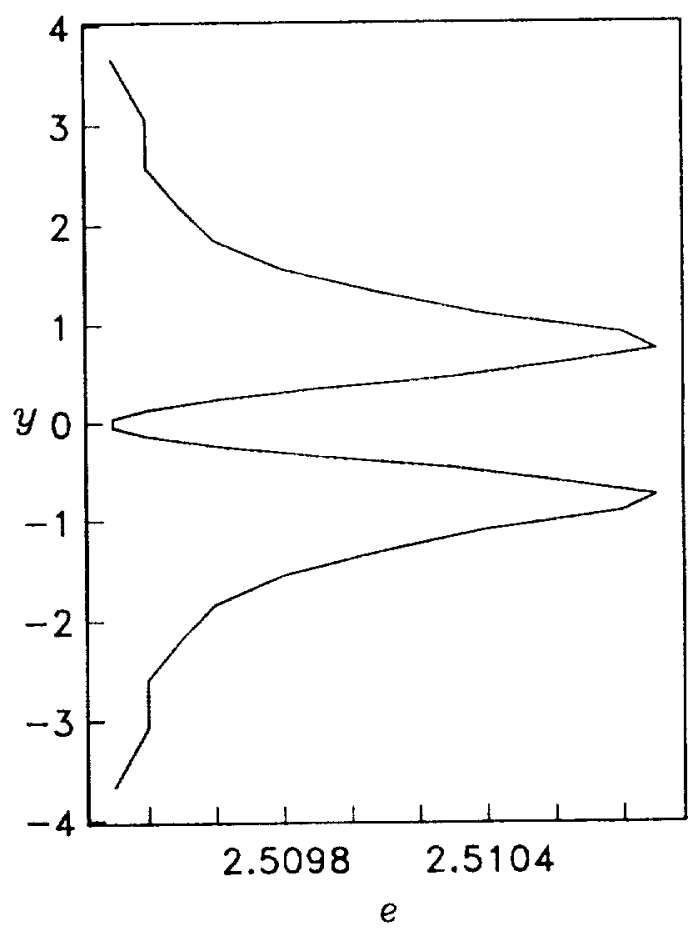

Figure 10-2. Energy Profile 


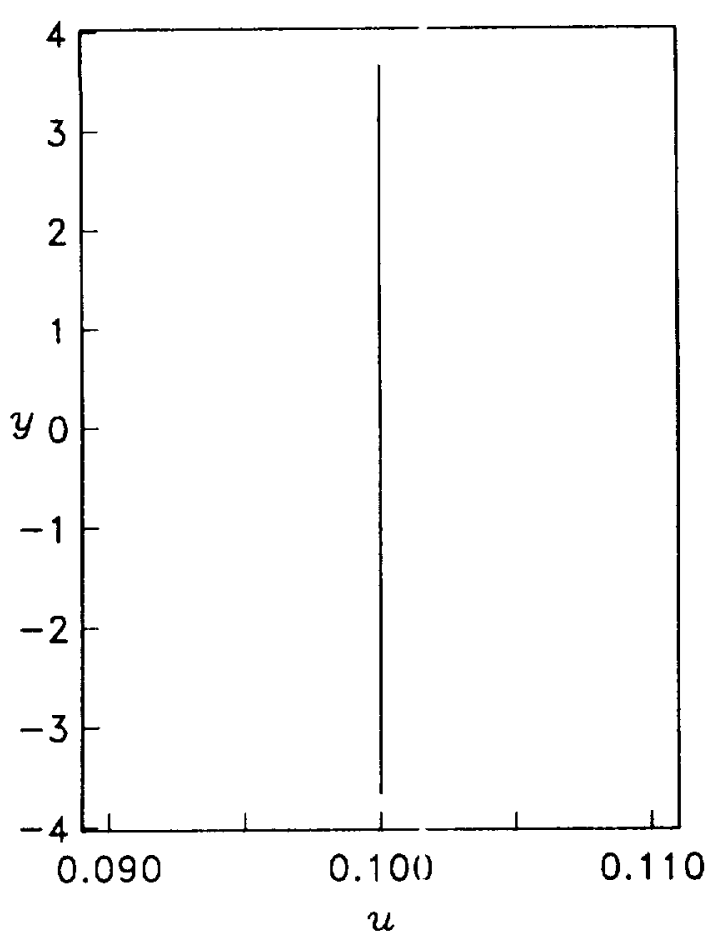

Figure 10-3. $u$-velocity Profile

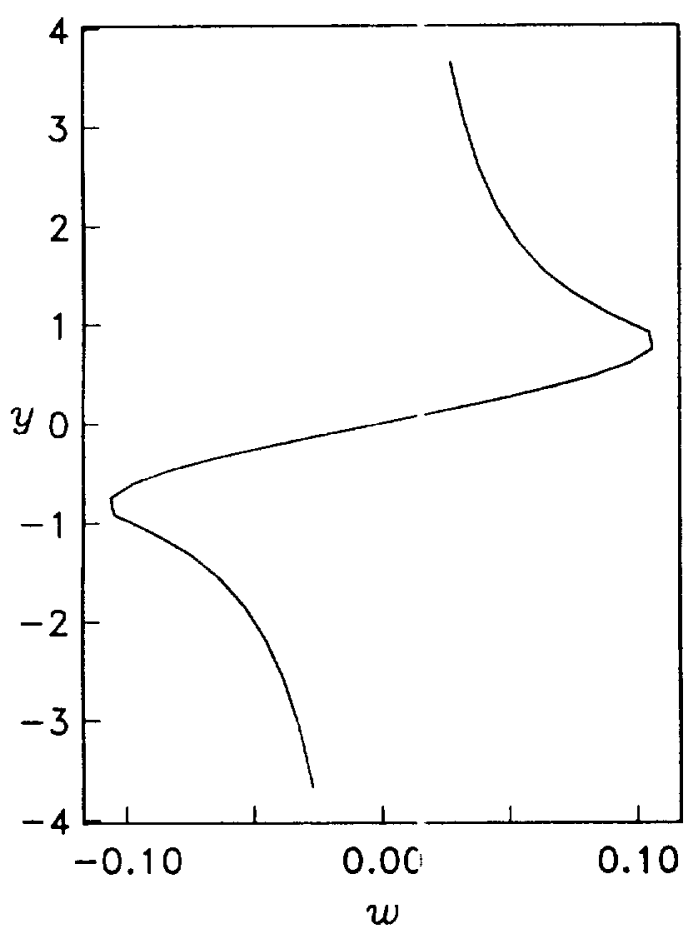

Figure 10-5. $w$-velocity Profile

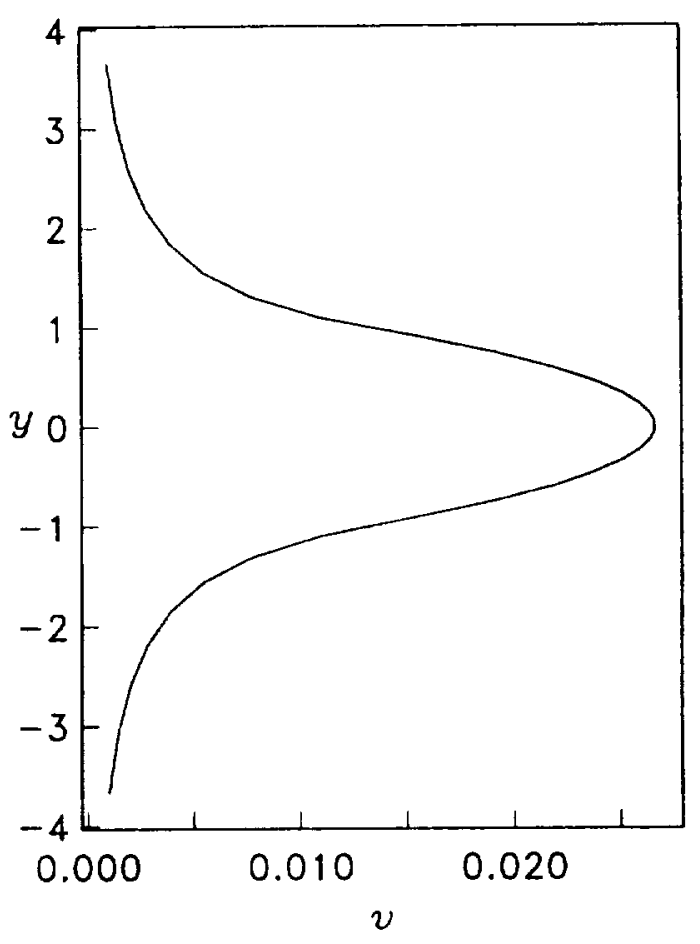

Figure 10-4. $v$-velocity Profile

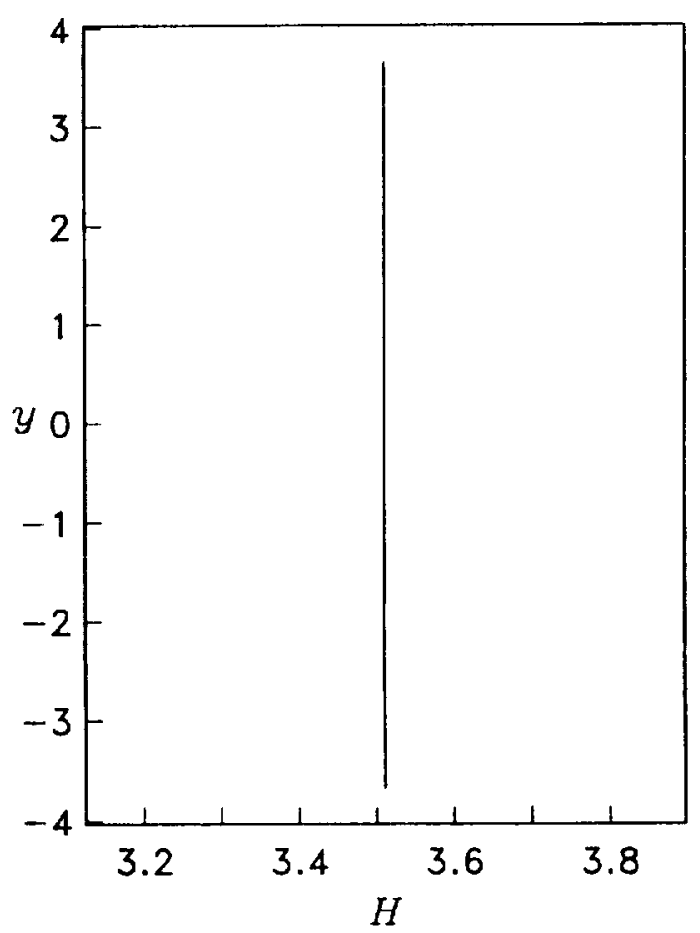

Figure 10-6. Total Enthalpy Profile 


\section{Results}

The cases that are presented here were run either on the Numerical Aerodynamic Simulator (NAS) Cray-2, called Navier, located at NASA Ames Research Center or on the Cray-2 called Voyager located at NASA Langley Research Center. The CPU time shown are normalized with respect to the Voyager CPU clock.

All the solutions shown have a reference Mach no. $M_{\infty}=0.1$. The computational domain, based on the vortex-core radius, is $10 \times 8 \times 8$ in the $x, y$ and $z$ directions respectively and there are $64 \times 32 \times 32$ cells in this domain with grid clustering around the vortex-axis. The boundaries around the vortex-axis (side-faces) are four vortex-core radii away from the $x$-axis. Fully upwind $(\kappa=-1)$ differencing with no limiting was used to discretize the inviscid fluxes. A three level $\mathrm{V}$-cycle multigrid was used to accelerate the convergence. A global-minimum time step with a $C F L=5$ was used to advance the solution in time. About 2500 multigrid cycles were required to drive the steady state residual to machine-zero.

Case 1: $R e=100, \mathcal{S}=1$ and $M_{\infty}=0.1$

Fig 11-1 shows the residual history of this case run with and without multigrid. It is clear that, by using multigrid, an additional six order of magnitude reduction in steady state residual was obtained in the same amount of time compared to the single grid calculation. Fig 11-2 shows the breakdown of the vortex. A tube of particles seeded around the vortex axis, near the incomingface, bulges around the breakdown region. A "tape" of particles seeded above the axis can be seen to follow the swirling flow, stretching and rolling-up as it travels downstream. It is clearly evident here that by using particle tubes and tapes, to visualize the flow, a lot more information about the flowfield can be obtained compared to tracking individual particles. This solution agrees, qualitatively, with calculations done in the past ${ }^{[22]}$ using an incompressible axisymmetric formulation. Figs. 11-3 to 11-8 shows the profiles of some of the flow variables at the incomingface along the $y$-direction at $(x, z: 0,0)$. These profiles are essentially axisymmetric. A slight asymmetry near the boundary can be noticed in the $v$-profile. This may be due to the boundary conditions on the side-faces. On the side faces, due to the swirl in the flowfield, one half of the face is treated as an inflow boundary while the other half is treated as an outflow boundary. Hence the entropy in the ghost cells on the inflow part of the face remains fixed at the initial level while the ghost cells in the outflow part of the face get updated with entropy from the interior of the flow. If the side faces are sufficiently far away from the vortex-axis the effect this should be negligible. Fig $11-9$ is a plot of the axial velocity along the $x$-axis. The region of negative velocities indicates the extend of the bubble. 


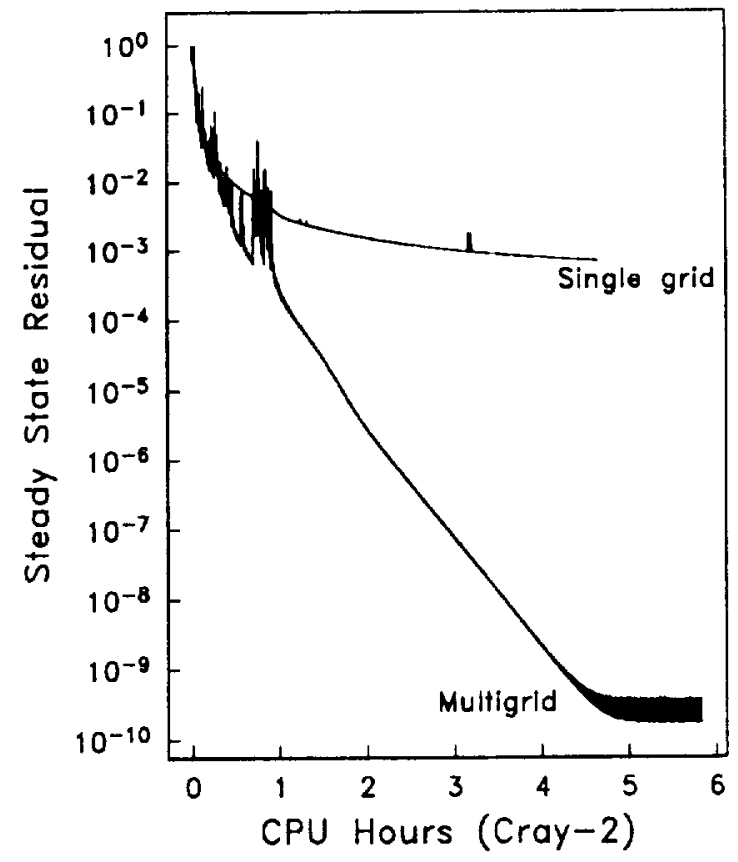

Figure 11-1. Residual History $\left(\operatorname{Re}=100, \mathcal{S}=1, M_{\infty}=0.1\right)$

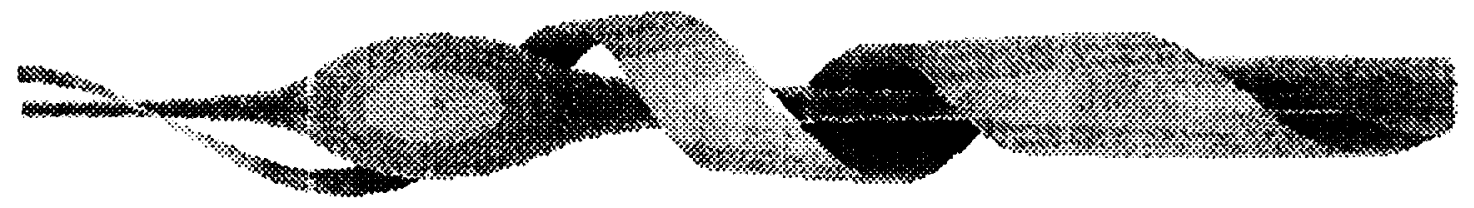

Figure 11-2. Streamsurfaces $\left(R e=100, \mathcal{S}=1, M_{\infty}=0.1\right)$ 


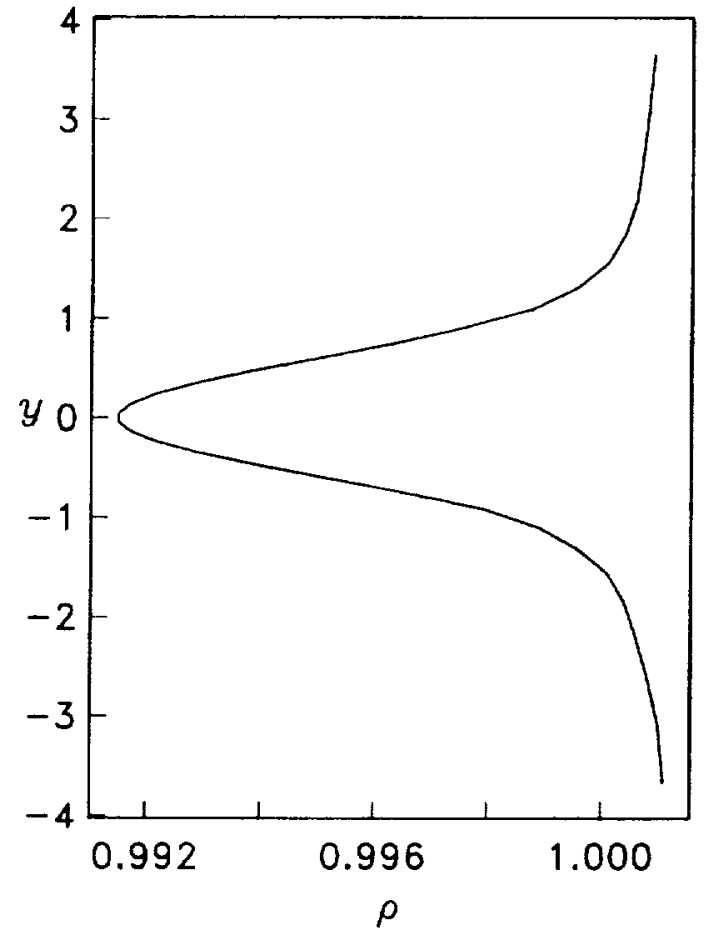

Figure 11-3. Density Profile

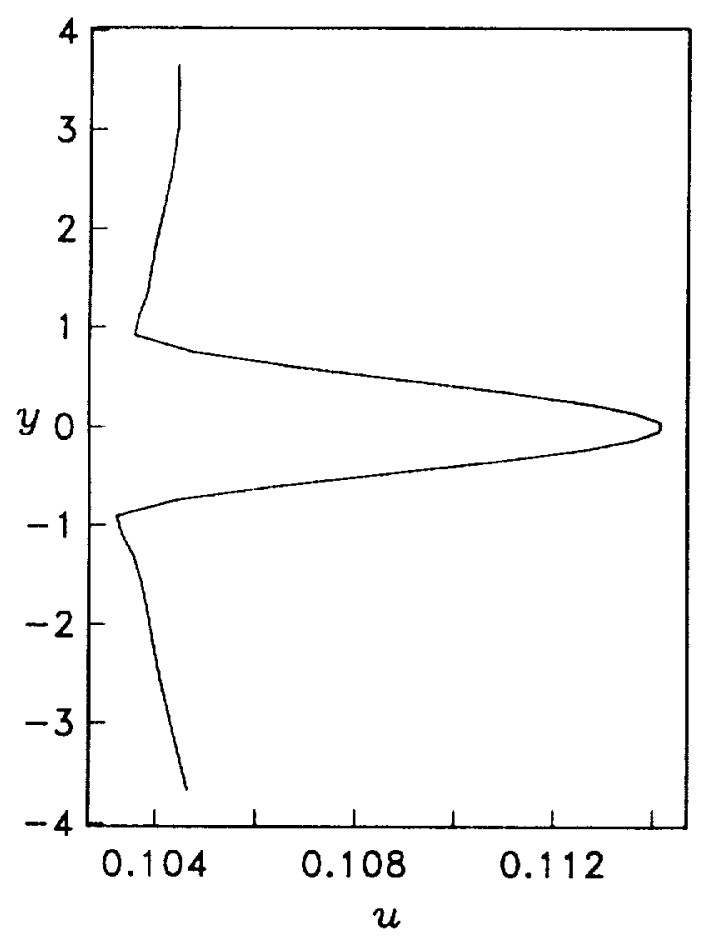

Figure 11-5. $u$-velocity Profile

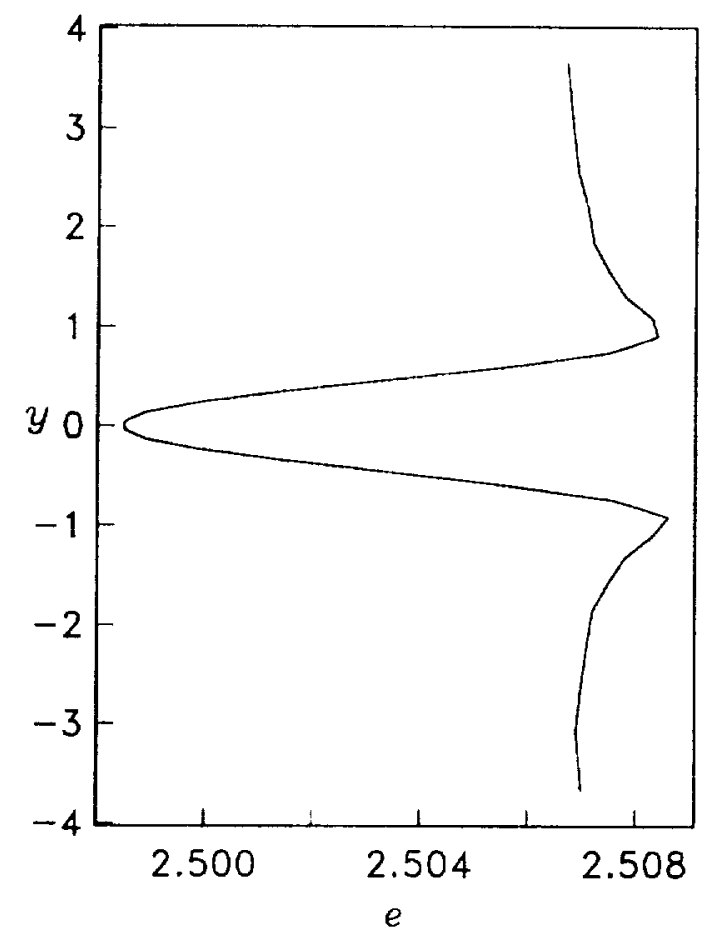

Figure 11-4. Energy Profile

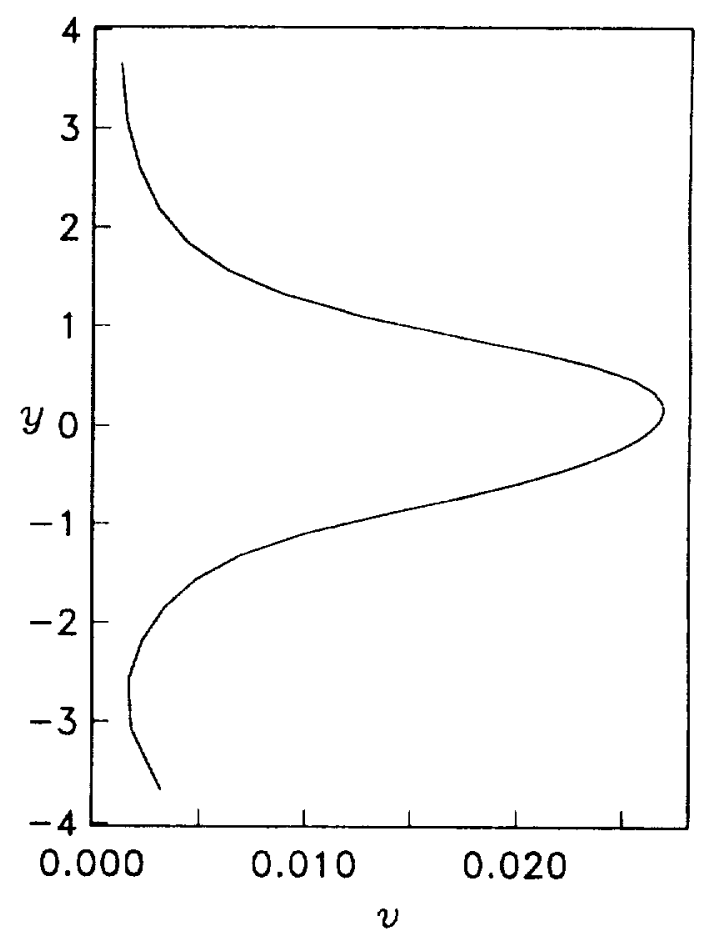

Figure 11-6. $v$-velocity Profile 


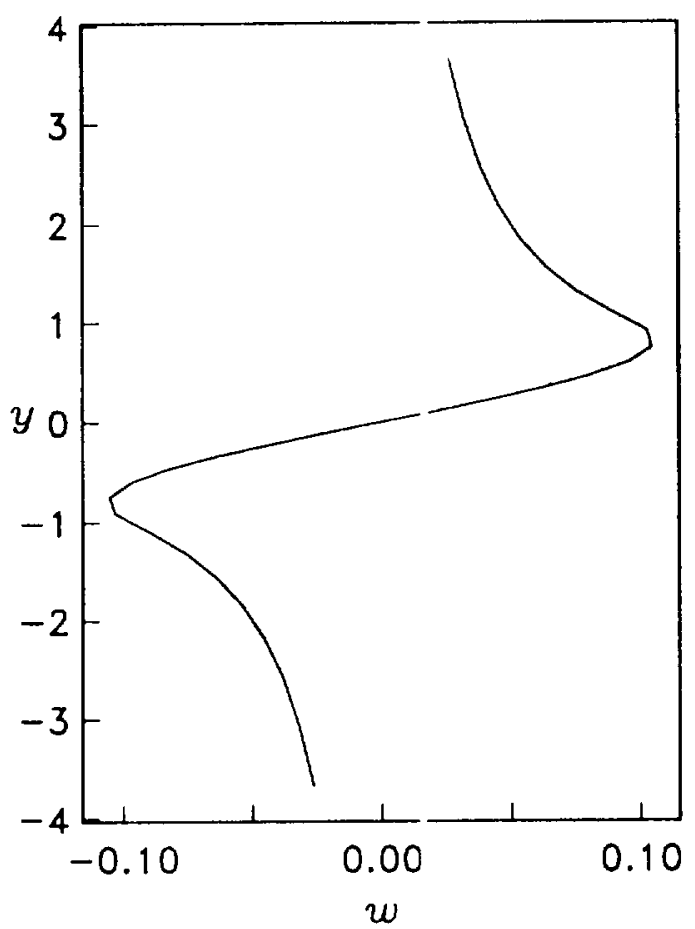

Figure 11-7. $w$-velocity Profile

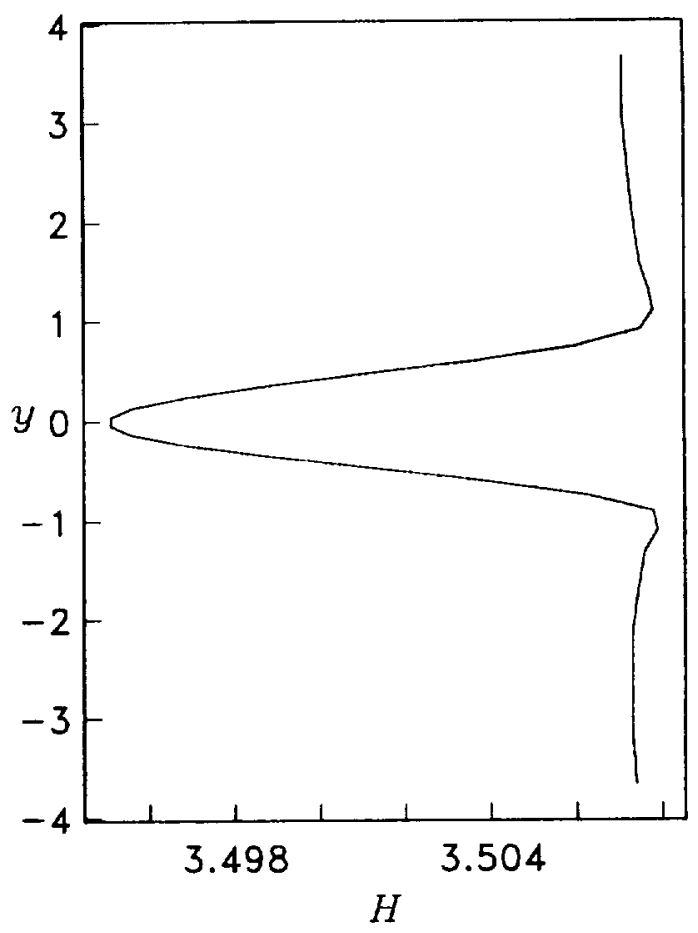

Figure 11-8. Total Enthalpy Profile

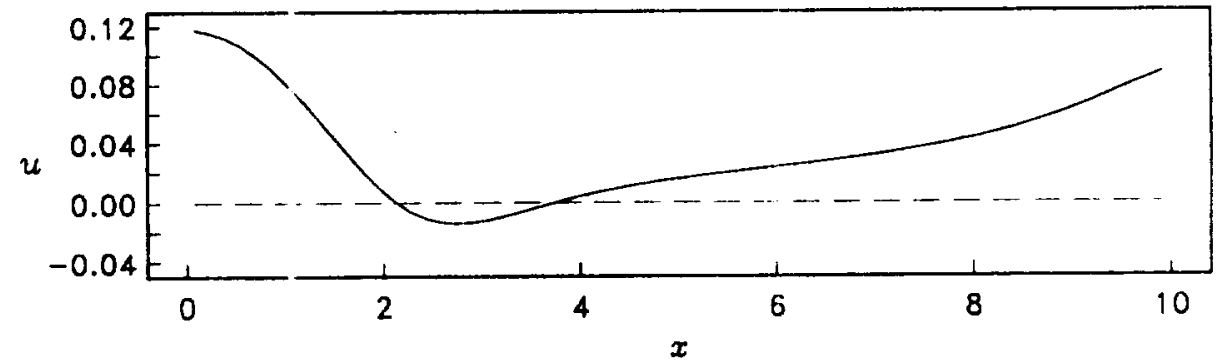

Figure 11-9. Axial-velocity Along the $x$-axis $\left(R e=100, \mathcal{S}=1, M_{\infty}=0.1\right)$ 
Case 2: $\operatorname{Re}=375, \mathcal{S}=1.464$ and $M_{\infty}=0.1$

Fig. 11-10 shows the steady state residual history for this case. Fig. 11-11 shows the streamsurfaces. In this case the particle tube around the breakdown region has a mushroom like shape. The recirculation bubble has become pinched at the axis and is nearly shaped like a toroid. This pinching of the bubble has been observed in the axisymmetric calculations done in the past by the present authors ${ }^{[2]}$. A particle tape seeded above the axis shows a more pronounced stretching and roll-up as the particles move downstream. Fig. 11-12 shows the axial-velocity along the $x$-axis and, due to the pinching of the bubble, the flow along the axis does not have any negative velocities. However the axial-velocity along a line in the $y$-direction, at $(x, z: 1.17,0)$, shown in Fig.11-13, has negative velocities indicating a recirculation zone.

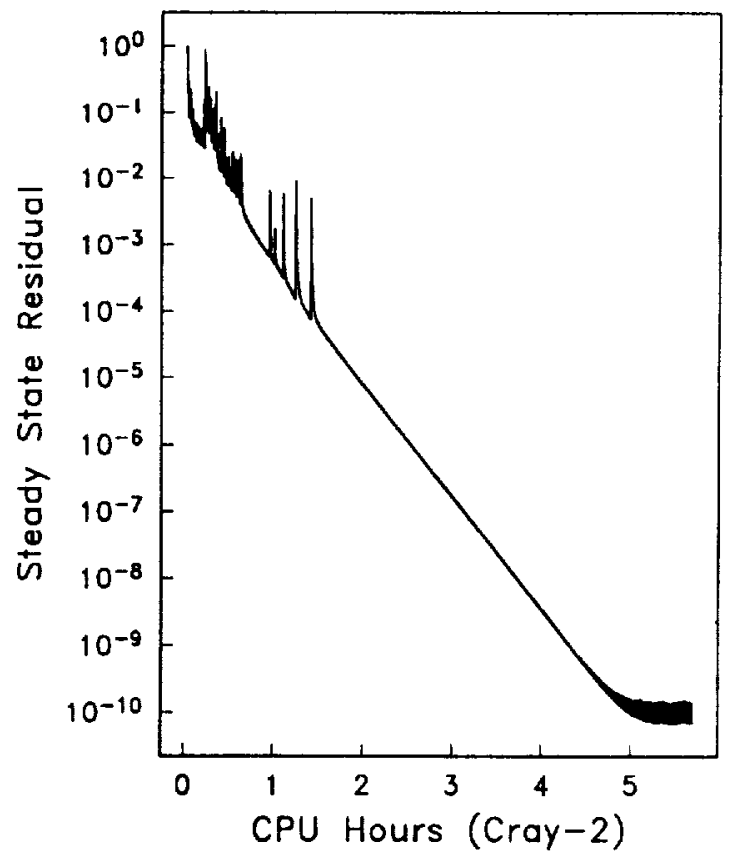

Figure 11-10. Residual History $\left(R e=375, \mathcal{S}=1.464, M_{\infty}=0.1\right)$ 


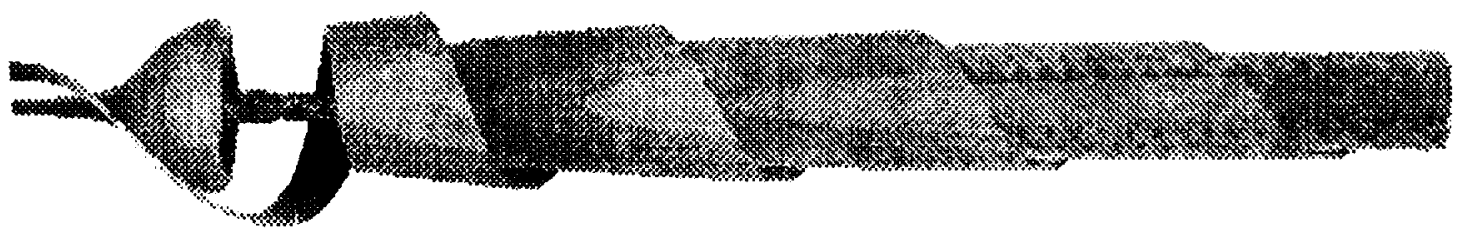

Figure 11.11. Streamsurfaces $\left(R e=375, \mathcal{S}=1.464, M_{\infty}=0.1\right)$

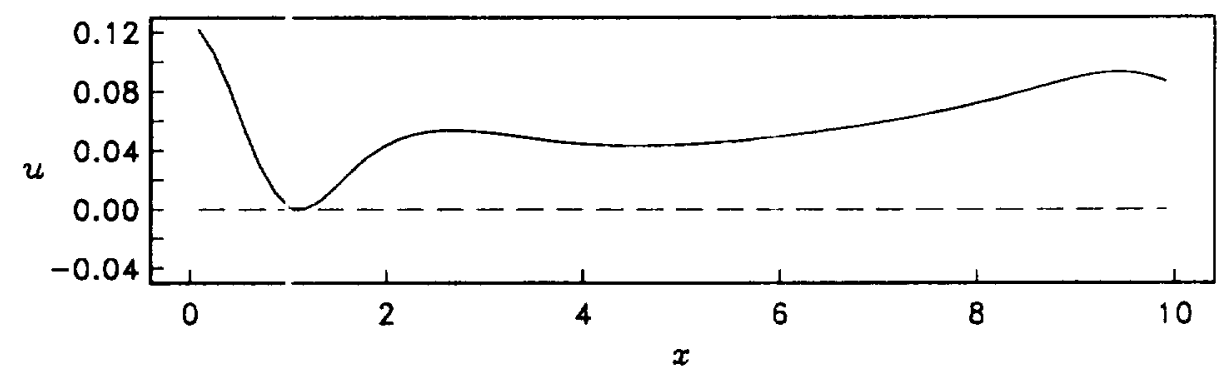

Figure 11-12. Axi:il-velocity Along the $x$-axis $\left(R e=375, \mathcal{S}=1.464, M_{\infty}=0.1\right)$

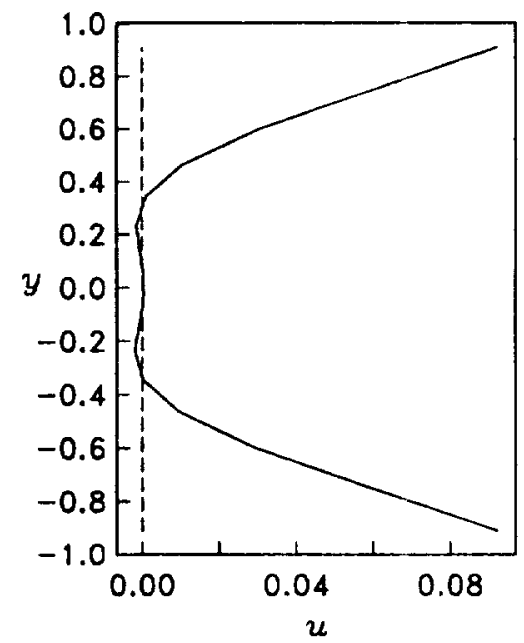

Figure 11-13. Axial-velocity Along $y(x, z: 1.17,0)\left(R e=375, \mathcal{S}=1.464, M_{\infty}=0.1\right)$ 
Case 3: $R e=400, \mathcal{S}=1$ and $M_{\infty}=0.1$

Fig. 11-14 shows the steady state residual history for this case. Fig. 11-15 shows the streamsurface and particle traces. In this case a set of particles seeded near the axis can be seen to go around the breakdown bubble. A particle seeded inside the bubble stays trapped inside the recirculation region. Again the observations are consistent with the ones seen in previous axisymmetric simulations. A particle tape seeded above the axis shows stretching and roll-up as the particles move downstream.

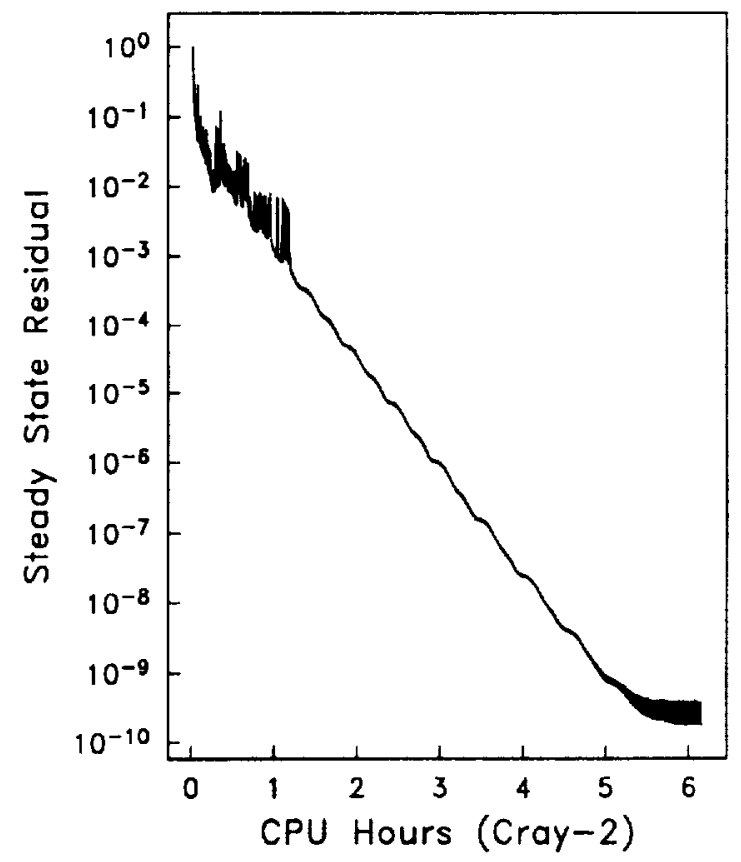

Figure 11-14. Residual History $\left(R e=400, \mathcal{S}=1, M_{\infty}=0.1\right)$

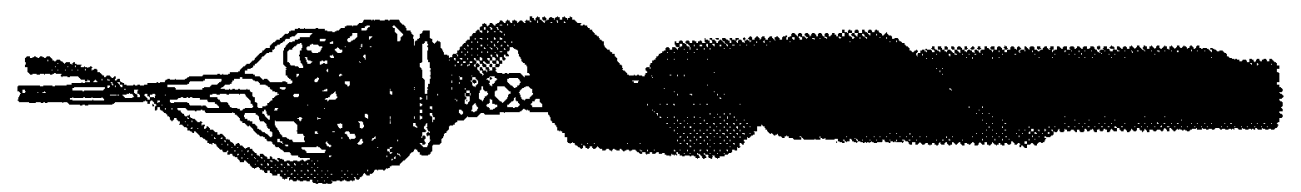

Figure 11-15. Particle Traces/Streamsurface $\left(R e=400, \mathcal{S}=1, M_{\infty}=0.1\right)$ 


\section{Concluding Remarks}

A three dimensional tinite volume full Navier-Stokes code has been developed to study the vortex breakdown phenomenon. The code is robust and its performance, both in terms of computer resources requirement and rate of convergence, is good.

The bubble-type breakdown has been simulated for several sets of Reynolds numbers and Swirl velocity parameters. The results agree, qualitatively, with the ones obtained in the past using an axisymmetric incompressible formulation. We believe, that this code can be used as a good platform to conduct further extensive numerical experiments and to investigate other types of breakdown. 


\section{Appendix A}

Consider a cell $i, j, k$ in the flowfield, shown in Fig. 3-3. Let $Q_{i, j, k}$ represent the conserved variables located at the cell-center. The semidiscrete finite-volume representation of the governing equations is (Eq. 3-4)

$$
\begin{aligned}
\left(\frac{1}{J} \frac{\partial Q}{\partial t}\right)_{i, j, k} & +(E-\mathcal{E})_{i+\frac{1}{2}, j, k}-(E-\mathcal{E})_{i-\frac{1}{2}, j, k} \\
& +(F-\mathcal{F})_{i, j+\frac{1}{2}, k}-(F-\mathcal{F})_{i, j-\frac{1}{2}, k} \\
& +(G-\mathcal{G})_{i, j, k+\frac{1}{2}}-(G-\mathcal{G})_{i, j, k-\frac{1}{2}}=0
\end{aligned}
$$

where the fluxes are evaluated at the cell interfaces defined by the grid points. Substituting Eqs. 224 and 2-25 we get

$$
\begin{aligned}
\left(\frac{1}{J} \frac{\partial Q}{\partial t}\right)_{i, j, k}+ & {\left[\left(\vec{F} \cdot \frac{\nabla \xi}{|\nabla \xi|}\right) \frac{|\nabla \xi|}{J}\right]_{i+\frac{1}{2}, j, k}-\left[\left(\vec{F} \cdot \frac{\nabla \xi}{|\nabla \xi|}\right) \frac{|\nabla \xi|}{J}\right]_{i-\frac{1}{2}, j, k} } \\
+ & {\left[\left(\vec{F} \cdot \frac{\nabla \eta}{|\nabla \eta|}\right) \frac{|\nabla \eta|}{J}\right]_{i, j+\frac{1}{2}, k}-\left[\left(\vec{F} \cdot \frac{\nabla \eta}{|\nabla \eta|}\right) \frac{|\nabla \eta|}{J}\right]_{i, j-\frac{1}{2}, k} } \\
+ & {\left[\left(\vec{F} \cdot \frac{\nabla \zeta}{|\nabla \zeta|}\right) \frac{|\nabla \zeta|}{J}\right]_{i, j, k+\frac{1}{2}}-\left[\left(\vec{F} \cdot \frac{\nabla \zeta}{|\nabla \zeta|}\right) \frac{|\nabla \zeta|}{J}\right]_{i, j, k-\frac{1}{2}}=0 }
\end{aligned}
$$

where

$$
\vec{F}=(\tilde{E}-\tilde{\mathcal{E}}) \hat{i}+(\tilde{F}-\tilde{\mathcal{F}}) \hat{j}+(\tilde{G}-\tilde{\mathcal{G}}) \hat{k}
$$

It can be shown that the terms $\xi_{x} /|\nabla \xi|, \xi_{y} /|\nabla \xi|, \xi_{z} /|\nabla \xi|$ are the components of the unit normal vector, pointing in the $\xi$-direction, from the cell face that is normal to the $\xi$-direction. $|\nabla \xi| / J$ is the directed area of the cell-face and $1 / J$ is the volume bounded by the cell-interfaces. Hence Eq. A-2 can be written as 


$$
\begin{aligned}
\left(\frac{1}{J} \frac{\partial Q}{\partial t}\right)_{i, j, k} & +[(\vec{F} \cdot \hat{n})|\Delta S|]_{i+\frac{1}{2}, j, k}+[(\vec{F} \cdot \hat{n})|\Delta S|]_{i-\frac{1}{2}, j, k} \\
& +[(\vec{F} \cdot \hat{n})|\Delta S|]_{i, j+\frac{1}{2}, k}+[(\vec{F} \cdot \hat{n})|\Delta S|]_{i, j-\frac{1}{2}, k} \\
& +[(\vec{F} \cdot \hat{n})|\Delta S|]_{i, j, k+\frac{1}{2}}+[(\vec{F} \cdot \hat{n})|\Delta S|]_{i, j, k-\frac{1}{2}}=0
\end{aligned}
$$

where

$$
\hat{n}=n_{x} \hat{i}+n_{y} \hat{j}+n_{z} \hat{k}
$$

is the unit normal vector pointing outward from the cell-face and $|\Delta S|$ is the cell-face area. If the conserved variables $Q_{i, j, k}$ is regarded as a cell-averaged value, i.e.,

$$
Q_{i, j, k}^{n}=\left[\frac{1}{V} \iiint_{V} Q(x, y, z, n \Delta t)_{i, j, k} d V\right]
$$

where $V$ is the cell volume, Eq. A-4 can be written in the integral form as

$$
\frac{\partial}{\partial t} \iiint_{V} Q d V+\iint_{S} \vec{F} \cdot \hat{n} d S=0
$$

This is the integral form of the conservation equations. Hence Eq. 3-4 is a consistent approximation to the integral form of the conservation equations. 


\section{Appendix B}

The matrices $T_{r} \Pi_{r}^{+} T_{r}^{-1}$ and $T_{r} \Pi_{r}^{-} T_{r}^{-1}$ are the same as the matrix $T_{r} \Lambda_{r} T_{r}^{-1}$ except that they are evaluated using Roe-averaged quantities and the eigenvalue matrix contain only either the positive or negative eigenvalues. In order to evaluate the matrix $\mathbf{T}_{r} \boldsymbol{\Lambda}_{r} \mathbf{T}_{r}^{-1}$ we need to know the tangential components of the velocity $\bar{v}$ and $\bar{w}$ that are parallel the unit vectors $\bar{l}$ and $\bar{m}$. However the choice of $\bar{l}$ and $\bar{m}$ is arbitrary. The arbitrariness in choosing the tangential vectors can be avoided in the following way. By definition we can write

$$
\left[\begin{array}{c}
\bar{u} \\
\bar{v} \\
\bar{w}
\end{array}\right]=\left[\begin{array}{ccc}
\bar{r}_{x} & \bar{r}_{y} & \bar{r}_{z} \\
\bar{l}_{x} & \bar{l}_{y} & \bar{l}_{z} \\
\bar{m}_{x} & \bar{m}_{y} & \bar{m}_{z}
\end{array}\right]\left[\begin{array}{c}
u \\
v \\
w
\end{array}\right]
$$

Since $\bar{r}, \bar{l}$ and $\bar{m}$ are mutually perpendicular unit vectors the $3 \times 3$ matrix in Eq. B-1 is an orthogonal matrix. Hence its inverse is the same as its transpose, i.e.,

$$
\left[\begin{array}{ccc}
\bar{r}_{x} & \bar{l}_{x} & \bar{m}_{x} \\
\bar{r}_{y} & \bar{l}_{y} & \bar{m}_{y} \\
\bar{r}_{z} & \bar{l}_{z} & \bar{m}_{z}
\end{array}\right]\left[\begin{array}{ccc}
\bar{r}_{x} & \bar{r}_{y} & \bar{r}_{z} \\
\bar{l}_{x} & \bar{l}_{y} & \bar{l}_{z} \\
\bar{m}_{x} & \bar{m}_{y} & \bar{m}_{z}
\end{array}\right]=\mathbf{I}
$$

Therefore we can write

$$
\left[\begin{array}{c}
u \\
v \\
w
\end{array}\right]=\left[\begin{array}{ccc}
\bar{r}_{x} & \bar{l}_{x} & \bar{m}_{x} \\
\bar{r}_{y} & \bar{l}_{y} & \bar{m}_{y} \\
\bar{r}_{z} & \bar{l}_{z} & \bar{m}_{z}
\end{array}\right]\left[\begin{array}{c}
\bar{u} \\
\bar{v} \\
\bar{w}
\end{array}\right]
$$

From Eq. B-3 we get

$$
\begin{aligned}
& \bar{v} \bar{l}_{x}+\bar{w} \bar{m}_{x}=u-\bar{u} \bar{r}_{x} \\
& \bar{v} \bar{l}_{y}+\bar{w} \bar{m}_{y}=v-\bar{u} \bar{r}_{y} \\
& \bar{v} \bar{l}_{z}+\bar{w} \bar{m}_{z}=w-\bar{u} \bar{r}_{z}
\end{aligned}
$$

Eq. B-4

Using Eqs. B-2 and B-4 and from the fact that

$$
\frac{\bar{u}^{2}+\bar{v}^{2}+\bar{w}^{2}}{2}=q^{2}
$$

the columns of matrix $\mathbf{T}_{r} \Lambda_{r} \mathbf{T}_{r}^{-1}$ can be written as follows. 


$$
\begin{aligned}
& \text { Column } 1= {\left[\begin{array}{c}
\sigma q^{2} \lambda_{r 1}-\bar{u} \lambda_{r 2}+\lambda_{r 3} \\
\left\{\sigma q^{2} H-a^{2} \bar{u}^{2}\right\} \lambda_{r 1}+\bar{u}\left\{\sigma q^{2}-H\right\} \lambda_{r 2} \\
\left\{\sigma q^{2} u-a^{2} \bar{u}_{x}\right\} \lambda_{r 1}+\left\{\sigma q^{2} \bar{r}_{x}-\bar{u} u\right\} \lambda_{r 2} \\
\left\{\sigma q^{2} v-a^{2} \bar{u}_{y}\right\} \lambda_{r 1}+\left\{\sigma q^{2} \bar{r}_{y}-\bar{u} v\right\} \lambda_{r 2} \\
\left\{\sigma q^{2} w-a^{2} \bar{u} \bar{r}_{z}\right\} \lambda_{r 1}+\left\{\sigma q^{2} \bar{r}_{z}-\bar{u} w\right\} \lambda_{r 2}
\end{array}\right] } \\
& \text { Column } 2=\left[\begin{array}{c}
\sigma \lambda_{r 1} \\
\sigma H \lambda_{r 1}+\sigma \bar{u} \lambda_{r 2}+\lambda_{r 3} \\
\sigma u \lambda_{r 1}+\sigma \bar{r}_{x} \lambda_{r 2} \\
\sigma v \lambda_{r 1}+\sigma \bar{r}_{y} \lambda_{r 2} \\
\sigma w \lambda_{r 1}+\sigma \bar{r}_{z} \lambda_{r 2}
\end{array}\right] {\left[\begin{array}{c}
-\sigma u \lambda_{r 1}+\bar{r}_{x} \lambda_{r 2} \\
\left\{a^{2} \bar{u}_{x}-\sigma u H\right\} \lambda_{r 1}+\left\{H \bar{r}_{x}-\sigma u \bar{u}\right\} \lambda_{r 2} \\
\left\{a^{2} \bar{r}_{x} \bar{r}_{x}-\sigma u u\right\} \lambda_{r 1}+\left\{\bar{r}_{x} u-\sigma u \bar{r}_{x}\right\} \lambda_{r 2}+\lambda_{r 3} \\
\left\{a^{2} \bar{r}_{x} \bar{r}_{y}-\sigma u v\right\} \lambda_{r 1}+\left\{\bar{r}_{x} v-\sigma u \bar{r}_{y}\right\} \lambda_{r 2} \\
\left\{a^{2} \bar{r}_{x} \bar{r}_{z}-\sigma u w\right\} \lambda_{r 1}+\left\{\bar{r}_{x} w-\sigma u \bar{r}_{z}\right\} \lambda_{r 2}
\end{array}\right.} \\
& \text { Column } 3= \\
& \text { Column } 4=\left[\begin{array}{c}
-\sigma v \lambda_{r 1}+\bar{r}_{y} \lambda_{r 2} \\
\left\{a^{2} \bar{u}_{y}-\sigma v H\right\} \lambda_{r 1}+\left\{H \bar{r}_{y}-\sigma v \bar{u}\right\} \lambda_{r 2} \\
\left\{a^{2} \bar{r}_{y} \bar{r}_{x}-\sigma v u\right\} \lambda_{r 1}+\left\{\bar{r}_{y} u-\sigma v \bar{r}_{x}\right\} \lambda_{r 2} \\
\left\{a^{2} \bar{r}_{y} \bar{r}_{y}-\sigma v v\right\} \lambda_{r 1}+\left\{\bar{r}_{y} v-\sigma v \bar{r}_{y}\right\} \lambda_{r 2}+\lambda_{r 3} \\
\left\{a^{2} \bar{r}_{y} \bar{r}_{z}-\sigma v w\right\} \lambda_{r 1}+\left\{\bar{r}_{y} w-\sigma v \bar{r}_{z}\right\} \lambda_{r 2}
\end{array}\right] \\
& {\left[\begin{array}{c}
\left\{a^{2} \bar{u} \bar{r}_{z}-\sigma w H\right\} \lambda_{r 1}+\left\{H \bar{r}_{z}-\sigma w \bar{u}\right\} \lambda_{r 2} \\
\left\{a^{2} \bar{r}_{z} \bar{r}_{x}-\sigma w u\right\} \lambda_{r 1}+\left\{\bar{r}_{z} u-\sigma w \bar{r}_{x}\right\} \lambda_{r 2} \\
\left\{a^{2} \bar{r}_{z} \bar{r}_{y}-\sigma w v\right\} \lambda_{r 1}+\left\{\bar{r}_{z} v-\sigma w \bar{r}_{y}\right\} \lambda_{r 2} \\
\left\{a^{2} \bar{r}_{z} \bar{r}_{z}-\sigma w w\right\} \lambda_{r 1}+\left\{\bar{r}_{z} w-\sigma w \bar{r}_{z}\right\} \lambda_{r 2}+\lambda_{r 3}
\end{array}\right] }
\end{aligned}
$$

where

$$
\begin{aligned}
& \lambda_{r 1}=\left(\lambda_{r}^{+}+\lambda_{r}^{-}-2 \lambda_{r}^{0}\right) / 2 a^{2} \\
& \lambda_{r 2}=\left(\lambda_{r}^{+}-\lambda_{r}^{-}\right) / 2 a \\
& \lambda_{r 3}=\lambda_{r}^{0}
\end{aligned}
$$

Note that by substituting for $\lambda_{r}^{+}, \lambda_{r}^{-}$and $\lambda_{r}^{0}$ from Eq. 5-47, we can recover the matrix $\mathbf{K}_{r}$ given by Eq. 5-39.

From Eq. B-6 the matrices $T_{r} \Pi_{r}^{+} T_{r}^{-1}$ and $T_{r} \Pi_{r}^{-} T_{r}^{-1}$ can be evaluated by replacing the flow variables by their Roe-averages and by setting the negative and positive eigenvalues in $\Pi_{r}$ to zero respectively. The evaluation of $\Pi_{r}^{+}$and $\Pi_{r}^{-}$can be automated by using the following equations 


$$
\begin{aligned}
& \Pi_{r}^{+}=\frac{\Pi_{r}+\left|\Pi_{r}\right|}{2} \\
& \Pi_{r}^{-}=\frac{\Pi_{r}-\left|\Pi_{r}\right|}{2}
\end{aligned}
$$




\section{Appendix C}

Carrying out the linearization procedure outlined in section 6, we can write the viscous flux Jacobian matrix as

$$
\mathbf{V}_{r}:=\frac{|\nabla r|}{J} \frac{M_{\infty}}{R e} \mu\left[\begin{array}{ccccc}
0 & 0 & 0 & 0 & 0 \\
\Psi_{21 r} & \Psi_{22 r} & \Psi_{23 r} & \Psi_{24 r} & \Psi_{25 r} \\
\Psi_{31 r} & 0 & \Psi_{33 r} & \Psi_{34 r} & \Psi_{35 r} \\
\Psi_{41 r} & 0 & \Psi_{43 r} & \Psi_{44 r} & \Psi_{45 r} \\
\Psi_{51 r} & 0 & \Psi_{53 r} & \Psi_{54 r} & \Psi_{55 r}
\end{array}\right]
$$

where

$$
\begin{aligned}
& \Psi_{31 r}=-\phi_{1 r r} \frac{u}{\rho}-\phi_{2 r r} \frac{v}{\rho}-\phi_{3 r r} \frac{w}{\rho} \\
& \Psi_{33 r}=\frac{\phi_{1 r r}}{\rho} \\
& \Psi_{34 r}=\frac{\phi_{2 r r}}{\rho} \\
& \Psi_{35 r}=\frac{\phi_{3 r r}}{\rho} \\
& \Psi_{41 r}=-\phi_{4 r r} \frac{u}{\rho}-\phi_{5 r r} \frac{v}{\rho}-\phi_{6 r r} \frac{w}{\rho} \\
& \Psi_{43 r}=\frac{\phi_{4 r r}}{\rho} \\
& \Psi_{44 r}=\frac{\phi_{5 r r}}{\rho} \\
& \Psi_{45 r}=\frac{\phi_{6 r r}}{\rho} \\
& \Psi_{51 r}=-\phi_{7 r r} \frac{u}{\rho}-\phi_{8 r r} \frac{v}{\rho}-\phi_{9 r r} \frac{w}{\rho} \\
& \Psi_{53 r}=\frac{\phi_{7 r r}}{\rho} \\
& \Psi_{54 r}=\frac{\phi_{8 r r}}{\rho} \\
& \Psi_{55 r}=\frac{\phi_{9 r r}}{\rho}
\end{aligned}
$$




$$
\begin{aligned}
& \Psi_{21 r}=\Psi_{31 r} u+\Psi_{41 r} v+\Psi_{51 r} w-\frac{\phi_{0 r r} \gamma}{P_{r}} \frac{\left(e-2 q^{2}\right)}{\rho} \\
& \Psi_{22 r}=\frac{\phi_{0 r r} \gamma}{P_{r}} \frac{1}{\rho} \\
& \Psi_{23 r}=\Psi_{33 r} u+\Psi_{43 r} v+\Psi_{53 r} w-\frac{\phi_{0 r r} \gamma}{P_{r}} \frac{u}{\rho} \\
& \Psi_{24 r}=\Psi_{34 r} u+\Psi_{44 r} v+\Psi_{54 r} w-\frac{\phi_{0 r r} \gamma}{P_{r}} \frac{v}{\rho} \\
& \Psi_{25 r}=\Psi_{35 r} u+\Psi_{45 r} v+\Psi_{55 r} w-\frac{\phi_{0 r r} \gamma}{P_{r}} \frac{w}{\rho}
\end{aligned}
$$

and $r$ stands for $\xi, \eta$ or $\zeta$. Using Eqs. 2-25, 6-7, 6-8, 6-9 and 6-10 in Eqs. C-2, C-3, C-4 and C-5 we can write

$$
\begin{aligned}
& \Psi_{31 r}=J \frac{|\nabla r|}{J} \frac{1}{\rho}\left\{-u-\frac{1}{3} \bar{r}_{x}\left(u \bar{r}_{x}+v \bar{r}_{y}+w \bar{r}_{z}\right)\right\} \\
& \Psi_{33 r}=J \frac{|\nabla r|}{J} \frac{1}{\rho}\left\{1+\frac{1}{3} \bar{r}_{x} \bar{r}_{x}\right\} \\
& \Psi_{34 r}=J \frac{|\nabla r|}{J} \frac{1}{\rho}\left\{\frac{1}{3} \bar{r}_{x} \bar{r}_{y}\right\} \\
& \Psi_{35 r}=J \frac{|\nabla r|}{J} \frac{1}{\rho}\left\{\frac{1}{3} \bar{r}_{x} \bar{r}_{z}\right\} \\
& \Psi_{41 r}=J \frac{|\nabla r|}{J} \frac{1}{\rho}\left\{-v-\frac{1}{3} \bar{r}_{y}\left(u \bar{r}_{x}+v \bar{r}_{y}+w \bar{r}_{z}\right)\right\} \\
& \Psi_{43 r}=J \frac{|\nabla r|}{J} \frac{1}{\rho}\left\{\frac{1}{3} \bar{r}_{y} \bar{r}_{x}\right\} \\
& \Psi_{44 r}=J \frac{|\nabla r|}{J} \frac{1}{\rho}\left\{1+\frac{1}{3} \bar{r}_{y} \bar{r}_{y}\right\} \\
& \Psi_{45 r}=J \frac{|\nabla r|}{J} \frac{1}{\rho}\left\{\frac{1}{3} \bar{r}_{y} \bar{r}_{z}\right\} \\
& \Psi_{51 r}=J \frac{|\nabla r|}{J} \frac{1}{\rho}\left\{-w-\frac{1}{3} \bar{r}_{y}\left(u \bar{r}_{x}+v \bar{r}_{y}+w \bar{r}_{z}\right)\right\} \\
& \Psi_{53 r}=J \frac{|\nabla r|}{J} \frac{1}{\rho}\left\{\frac{1}{3} \vec{r}_{z} \bar{r}_{x}\right\} \\
& \Psi_{54 r}=J \frac{|\nabla r|}{J} \frac{1}{\rho}\left\{\frac{1}{3} \bar{r}_{z} \bar{r}_{y}\right\} \\
& \Psi_{55 r}=J \frac{|\nabla r|}{J} \frac{1}{\rho}\left\{1+\frac{1}{3} \bar{r}_{z} \bar{r}_{z}\right\}
\end{aligned}
$$




$$
\begin{aligned}
& \Psi_{21 r}=J \frac{\nabla r \mid}{J} \frac{1}{\rho}\left\{-2 q^{2}-\frac{1}{3}\left(u \bar{r}_{x}+v \bar{r}_{y}+w \bar{r}_{z}\right)^{2}-\frac{\gamma}{P r}\left(e-2 q^{2}\right)\right\} \\
& \Psi_{22 r}=J \frac{\nabla r \mid}{J} \frac{1}{\rho}\left\{\frac{\gamma}{P r}\right\} \\
& \Psi_{23 r}=J \frac{\nabla r \mid}{J} \frac{1}{\rho}\left\{u+\frac{1}{3} \bar{r}_{x}\left(u \bar{r}_{x}+v \bar{r}_{y}+w \bar{r}_{z}\right)-\frac{\gamma u}{P r}\right\} \\
& \Psi_{24 r}=J \frac{|\nabla r|}{J} \frac{1}{\rho}\left\{v+\frac{1}{3} \bar{r}_{y}\left(u \bar{r}_{x}+v \bar{r}_{y}+w \bar{r}_{z}\right)-\frac{\gamma v}{P r}\right\} \\
& \Psi_{25 r}=J \frac{\nabla r \mid}{J} \frac{1}{\rho}\left\{w+\frac{1}{3} \bar{r}_{z}\left(u \bar{r}_{x}+v \bar{r}_{y}+w \bar{r}_{z}\right)-\frac{\gamma w}{P r}\right\}
\end{aligned}
$$

where

$$
q^{2}=\frac{u^{2}+v^{2}+w^{2}}{2}
$$

Substituting Eqs. C-6, C-7, C-8 and C-9 in Eq. C-1 we get the final form given in Eq. 6-25. 


\section{References}

[1] D. H. Peckham and S. A. Atkinson, "Preliminary results of low speed wind tunnel tests on a gothic wing of aspect ratio 1.0," Technical Report CP No. 508, Aeronautical Research Council, 1957.

[2] B. J. Elle, "An investigation at low speed of the flow near the apex of thin delta wings with sharp leading edges," Reports and Memoranda No. 3176, Aeronautical Research Council, 1958.

[3] B. J. Elle, "On the breakdown at high incidences of the leading-edge vortices on delta wings," Journal of The Royal Aeronautical Society, vol. 64, no. 596, pp. 491-493, 1960.

[4] H. Werle, "Sur l'eclatement des tourbillions d'apex d'une aile delta aux faibles vitnesses," La Recherche Aeronautique, pp. 23-30, Jan/Feb 1960.

[5] N. C. Lambourne and D. W. Bryer, "The bursting of leading-edge voritces - Some observarions and discussions of the phenomenon," Reports and Memoranda No. 3282, Aeronautical Research Council, 1961.

[6] D. Hummel and P. S. Srinivasan, "Vortex breakdown effects on the low speed aerodynamic characteristic of slender delta wings in symmetrical flow," Journal of The Royal Aeronautical Society, vol. 71, pp. 319-322, April 1966.

[7] D. J. Peake and M. Tobac, "On issues concerning flow separation and vortical flows in three dimensions," Tech. Rep. CP No. 342, AGARD. Aerodynamics of Vortical Type Flows in Three Dimensions, Paper 1.

[8] M. Escudier, "Vortex breakdown in technology and nature." von Karman Institute for Fluid Dynamics Lecture Series 1986-08, Introduction to Vortex Dynamics, May 1986.

[9] J. K. Harvey, "Some observations of vortex breakdown phenomenon," Journal of Fluid Mechanics, vol. 14, no. 4, pp. 585-592, 1962.

[10]T. B. Benjamin, "Theory of the vortex breakdown phenomenon," Journal of Fluid Mechanics, vol. 14, no. 4, pp. 593-629, 1962.

[11]M. G. Hall, "Vortex breakdown," Annual Review of FLuid Mechanics, vol. 4, pp. 195-218, 1972.

[12]S. Leibovich, "The structure of vortex breakdown," Annual Review of FLuid Mechanics, vol. 10, pp. 221-246, 1978.

[13]S. Leibovich, "Vortex stability and breakdown," AIAA Journal, vol. 22, pp. 1192-1206, 1984.

[14]W. J. Grabowski and S. A. Berger, "Solutions of the Navier-Stokes equations for vortex breakdown," Journal of Fliud Mechanics, vol. 75, pp. 525-544, 1976.

[15] M. Hafez, G. Kuruvila and M. D. Salas, "Numerical study of vortex breakdown," Applied Numerical Mathematics, vol. 2, pp. 291-302, 1986.

[16] E. Krause, X. G. Shi and P. M. Hartwich, "Computation of leading edge vortices," AIAA Computational Fluid Dynamics Conference, Danvers, Massachusetts, 1983. Paper No. 83-1907. 
[17]S. Menne, Rotationssymmetrische Wirbel in Achsparalleler Stromung. $\mathrm{PhD}$ thesis, RheinischWestfalisch Technisch Hochschule, Aachen, 1986.

[18] M. Hafez, J. Ahmad G. Kuruvila and M. D. Salas, "Vortex breakdown simulation," AIAA 19th Fluid Dynamics, Plasma Dynamics and Lasers Conference, Honolulu, Hawaii, 1987. Paper No. 87-1343.

[19] M. D. Salas and G. Kuruvila, "Vortex breakdown simulation: A circumspect study of the steady laminar axisymmetric model," The Symposium on Physical Aspects of Numerical Gas Dynamics, Farmingdale, New York, August 1987.

[20] P. S. Beran, "Numerical simulation of trailing vortex bursting," AIAA 19th Fluid Dynamics, Plasma Dynamics and Lasers Conference, Honolulu, Hawaii, 1987. Paper No. 87-1313.

[21] M. D. Salas and G. Kuruvila, "Numerical simulation of vortex breakdown using primitive variables," Proceedings of the International Conference on Computational Engineering Science, Atlanta, Georgia, April 1988.

[22] M. D. Salas and G. Kuruvila, "Study of three-dimensional effects on vortex breakdown," 16th Congress of the International Council of the Aeronautical Sciences, Jerusalem, Israel, August/September 1988.

[23] R. M. Beam and R. F. Warming, "An implicit factored scheme for the compressible NavierStokes equations," AJAA Journal, vol. 16, pp. 393-402, April 1978.

[24] R. W. Walters and J. L. Thomas, "Advances in upwind relaxation methods," in State-of-theart Surveys on Computational Mechanics (A. K. Noor, ed.), ch. 4, pp. 145-183, New York: ASME, 1989.

[25]P. L. Roe, "Characteristic-based schemes for the euler equations," Annual Review of Fluid Mechanics, vol. 18, pp. 337-365, 1986.

[26]B. van Leer, "Upwind-difference methods for aerodynamics problems governed by the euler equations," 15th AMS/SIAM Seminar on Large Scale Computations in Fluid Dynamics, La Jolla, California, June/July 1983.

[27]D. A. Anderson, J. C. Tannehill and R. H. Pletcher, Computational Fluid Mechanics and Heat Transfer. McGraw-Hill, 1984.

[28]P. L. Roe, "Approximate riemann solvers, parameter vectors and difference schemes," Journal of Computational Physics, vol. 43, pp. 357-372, October 1981.

[29]A. Harten, "High resolution schemes for hyperbolic conservation laws," research and development report, Courant Mathematics and Computing Laboratory, March 1982.

[30] B. Noble and J. W. Daniel, Applied Linear Algebra. Prentice-Hall, 1977.

[31] W. Kordulla and M. Vinokur, "Efficient computation of volume in flow predictions," AIAA Journal, vol. 21, pp. 917-918, June 1983.

[32] S. Menne, "Vortex breakdown in an axisymmetric flow," AIAA 26th Aerospace Sciences Meeting, Reno, Nevada, January 1988. Paper No. 88-0506. 
[33] J. L. Thomas and M. D. Salas, "Far-field boundary conditions for transonic lifting solutions to the euler equations," AIAA 23rd Aerospace Sciences Meeting, Reno, Nevada, January 1985. Paper No. 85-0020.

[34]N. D. Melson, "Vectorizable multigrid algorithms for transonic flow calculations," Master's thesis, George Washington University, Washington DC, 1985.

[35] A. Brandt , "Multi-level adaptive solutions to boundary-value problems," Mathematics of Computation, vol. 31, pp. 333-390, April 1977.

[36] R. Radespiel and R. Swanson, "An investigation of cell centered and cell vertex multigrid schemes for the navier-stokes equations," AIAA 27th Aerospace Sciences Meeting, Reno, Nevada, January 1989. Paper No. 89-0548. 


\begin{tabular}{|c|c|c|c|c|}
\hline Nush & \multicolumn{4}{|c|}{ Report Documèntation Page } \\
\hline $\begin{array}{l}\text { 1. Report No. } \\
\text { NASA TM-102664 }\end{array}$ & \multicolumn{2}{|c|}{ 2. Government Accession No. } & \multicolumn{2}{|c|}{ 3. Recipient's Catalog No. } \\
\hline \multicolumn{3}{|l|}{ 4. Title and Subtitle } & \multicolumn{2}{|l|}{$\begin{array}{l}\text { 5. Report Date } \\
\text { June } 1990\end{array}$} \\
\hline \multicolumn{3}{|c|}{ Three-dimensional Simulaticn of Vortex Breakdown } & \multicolumn{2}{|c|}{6 Performing Organization Code } \\
\hline \multicolumn{3}{|l|}{ 7. Author(s) } & \multicolumn{2}{|c|}{ 8. Performing Organization Repor No } \\
\hline \multicolumn{3}{|c|}{ G. Kuruvila and M. D. Salis } & \multicolumn{2}{|c|}{$\begin{array}{l}\text { 10. Work Unit No. } \\
505-60-01-01\end{array}$} \\
\hline \multirow{2}{*}{\multicolumn{3}{|c|}{$\begin{array}{l}\text { 9. Performing Organization Name and Addre: } \mathrm{s} \\
\text { NASA Langley Research Center } \\
\text { Hampton, Virginia } 23665-5225\end{array}$}} & \multicolumn{2}{|c|}{ 11. Contract or Grant No. } \\
\hline \multirow{2}{*}{\multicolumn{3}{|c|}{$\begin{array}{l}\text { 12. Sponsoring Agency Name and Address } \\
\text { National Aeronautics and Space Administration } \\
\text { Washington, DC 20546-000 l }\end{array}$}} & \multicolumn{2}{|c|}{$\begin{array}{l}\text { 13. Type of Repon and Period Covered } \\
\text { Technical Memorandum }\end{array}$} \\
\hline & & & \multicolumn{2}{|c|}{ 14. Sponsoring Agency Code } \\
\hline \multicolumn{5}{|l|}{ 15. Supplementary Notes } \\
\hline \multicolumn{5}{|l|}{ 16. Abstract } \\
\hline \multicolumn{5}{|c|}{$\begin{array}{l}\text { The integral form of the complete, unsteady, compressible, three-dimensional Navier-Stokes } \\
\text { equations in the conservation form, cast in generalized coordinate system, are solved, numerically, } \\
\text { to simulate the vortex breakulown phenomenon. The inviscid fluxes are discretized using Roe's } \\
\text { upwind-biased flux-difference splitting scheme and the viscous fluxes are discretized using } \\
\text { central differencing. Time integration is performed using a backward Euler ADI scheme. A full } \\
\text { approximation multigrid is used to accelerate the convergence to steady state. }\end{array}$} \\
\hline \multirow{2}{*}{\multicolumn{2}{|c|}{$\begin{array}{l}\text { 17. Key Words (Suggested by Author(s) } \\
\text { Vortex Breakdown, Numericil Simulation, } \\
\text { Roe's Flux-difference Splitting } \\
\text { ADI, Multigrid }\end{array}$}} & \multirow{2}{*}{\multicolumn{3}{|c|}{$\begin{array}{l}\text { 18. Distribution Statement } \\
\text { Unclassified-Unlimited } \\
\text { Subject Category } 02\end{array}$}} \\
\hline & & & & \\
\hline $\begin{array}{l}\text { 19. Security Classif. (of this report) } \\
\text { Unclassified }\end{array}$ & $\begin{array}{r}\text { 20. Security Classi } \\
\text { Unclassifi }\end{array}$ & is paget & $\begin{array}{c}\text { 21. No. of pages } \\
74\end{array}$ & $\begin{array}{r}\text { 22. Price } \\
\text { A04 }\end{array}$ \\
\hline
\end{tabular}


